\title{
Pseudolatirus Bellardi, 1884 revisited, with the description of two new genera and five new species (Neogastropoda: Fasciolariidae)
}

\author{
Yuri I. KANTOR ${ }^{1, *}$, Alexander E. FEDOSOV ${ }^{2}$, \\ Martin Avery SNYDER ${ }^{3} \&$ Philippe BOUCHET ${ }^{4}$ \\ ${ }^{1,2}$ A.N. Severtsov Institute of Ecology and Evolution, Russian Academy of Sciences, \\ Leninski prospect 33, 119071, Moscow, Russian Federation. \\ ${ }^{3,4}$ Institut de Systématique, Evolution, Biodiversité ISYEB - UMR 7205 - CNRS, MNHN, UPMC, \\ EPHE, Muséum national d'Histoire naturelle, Sorbonne Universités, \\ 57 rue Cuvier, CP26, F-75005, Paris, France. \\ ${ }^{3}$ Academy of Natural Sciences of Drexel University, 1900 Benjamin Franklin Parkway, \\ Philadelphia, PA 19103-1195, USA. \\ ${ }^{*}$ Corresponding author: kantor.yuri1956@gmail.com \\ 2Email: fedosovalexander@gmail.com \\ ${ }^{3}$ Email: dr.martin.snyder@gmail.com \\ ${ }^{4}$ Email: pbouchet@mnhn.fr

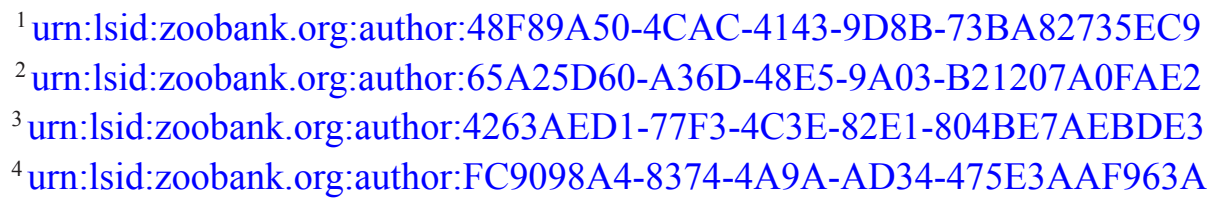

\begin{abstract}
The genus Pseudolatirus Bellardi, 1884, with the Miocene type species Fusus bilineatus Hörnes, 1853, has been used for 13 Miocene to Early Pleistocene fossil species and eight Recent species and has traditionally been placed in the fasciolariid subfamily Peristerniinae Tryon, 1880. Although the fossil species are apparently peristerniines, the Recent species were in their majority suspected to be most closely related to Granulifusus Kuroda \& Habe, 1954 in the subfamily Fusininae Wrigley, 1927. Their close affinity was confirmed by the molecular phylogenetic analysis of Couto et al. (2016). In the molecular phylogenetic section we present a more detailed analysis of the relationships of 10 Recent Pseudolatirus-like species, erect two new fusinine genera, Okutanius gen. nov. (type species Fusolatirus kuroseanus Okutani, 1975) and Vermeijius gen. nov. (type species Pseudolatirus pallidus Kuroda \& Habe, 1961). Five species are described as new for science, three of them are based on sequenced specimens (Granulifusus annae sp. nov., G. norfolkensis sp. nov., Okutanius ellenae gen. et sp. nov.) and two (G. tatianae sp. nov., G. guidoi sp. nov.) are attributed to Granulifusus on the basis of conchological similarities to sequenced species. New data on radular morphology is presented for examined species.
\end{abstract}

Keywords. Gastropod, Fasciolariidae, Pseudolatirus, molecular phylogeny, new genera, new species, Southwest Pacific. 
Kantor Y.I., Fedosov A.E., Snyder M.A. \& Bouchet P. 2018. Pseudolatirus Bellardi, 1884 revisited, with the description of two new genera and five new species (Neogastropoda: Fasciolariidae). European Journal of Taxonomy 433: 1-57. https://doi.org/10.5852/ejt.2018.433

\section{Introduction}

The species of Fasciolariidae inhabit warm temperate to tropical seas, mostly in intertidal and offshore subtidal depths, with some genera having radiated into deeper water (e.g., Granulifusus Kuroda \& Habe, 1954 and Amiantofusus Fraussen, Kantor \& Hadorn, 2007). Most fasciolariids form rather sparse populations and are usually represented in museum collections by lots with one or just a few specimens, making it difficult to assess the value of characters in terms of within-population and between-population variation. The taxonomy of Fasciolariidae up to present has been based mostly on shell and, to a lesser extent, radula characters. In many cases attribution of species to genera and genera to subfamilies is done by tradition, without an explicit discussion of the values of these characters. Only recently the phylogeny of the entire family was addressed from a molecular point of view, with the first multilocus molecular tree of Fasciolariidae (Couto et al. 2016), resulting in drastic changes in the composition of the three currently recognized subfamilies. In particular, the subfamily Peristerniinae Tryon, 1880 appeared to be restricted to Peristernia Mörch, 1852 (non-monophyletic) and Fusolatirus Kuroda \& Habe, 1971. Most of the formerly peristerniine genera ended up in Fasciolariinae Gray, 1853, while the Recent species assigned to Pseudolatirus Bellardi, 1884 ended up in Fusininae Wrigley, 1927. Six species referrable to Pseudolatirus on the basis of shell characters were included in the analysis of Couto et al. (2016) and they formed three separate clades, not closely related to each other. This result indicated that further molecular studies were needed to clarify the affinities of Pseudolatirus-like species, and consider their proper taxonomic treatment.

As conceived by Bellardi (1884), the genus Pseudolatirus comprised the type species (by subsequent designation by Cossmann 1901: 24) Fusus bilineatus Hörnes, 1853 (Miocene of central Europe) and seven additional Miocene and Pliocene European species: Latirus (Peristernia) cognatus Bellardi, 1884; Latirus (Pseudolatirus) concinnus Bellardi, 1884; Fasciolaria costata (Bellardi \& Michelotti, 1840) [renamed Fasciolaria subcostata by d'Orbigny (1852)]; Latirus (Pseudolatirus) fornicatus Bellardi, 1884; Latirus (Pseudolatirus) ligusticus Bellardi, 1884; Latirus (Pseudolatirus) pinensis Bellardi, 1884 and Pseudolathyrus rarocingulata (Foresti, 1876). Pseudolathyrus Cossmann, 1901, is an unjustified emendation and thus an objective synonym of Pseudolatirus. Subsequently, five Late Miocene to Pleistocene species from Indonesia and Japan were further included in Pseudolatirus: Pseudolathyrus burcki Koperberg, 1931; Pseudolathyrus esi Koperberg, 1931; Colus (?) teschi Finlay, 1927 (new name for Latirus fusiformis Tesch, 1915); Latirus gerthi Wanner \& Hahn, 1935 and Pseudolatirus yonabaruensis MacNeil, 1961 (Koperberg 1931; Finlay 1927; Wanner \& Hahn 1935; MacNeil 1961). Shutō (1969: 150) reported $P$. esi from the Neogene of Panay, Philippine Islands, and noted its similarity to . gerthi; he also reported P. teschi from the same deposits and noted that it was allied to P. burcki. Finally, MacNeil (1961: 83) noted that $P$. burcki, P. esi and P. teschi all resemble $P$. yonabaruensis described from the Late Miocene of Okinawa, a species subsequently reported by Robba et al. (1989: 80) from the Early Pleistocene of Timor (Indonesia). A careful examination of these species leads us to conclude that they probably all represent a single widely-distributed and variable species, which we attribute to Pseudolatirus and for which the valid name would be Pseudolatirus teschi (Finlay, 1927).

Seven Recent species are currently assigned to Pseudolatirus of which five were originally described as members of the genus: Pseudolatirus discrepans Kuroda \& Habe, 1961; Pseudolatirus pallidus Kuroda \& Habe, 1961; Pseudolatirus kurodai Okutani \& Sakurai, 1964; Fusolatirus kuroseanus Okutani, 1975; Pseudolatirus leucostriatus Kosuge, 1979; Pseudolatirus aikeni Lussi, 2014 and Pseudolatirus 
jeanpierrevezzaroi Cossignani, 2017. In the original descriptions the generic position of these species was neither justified nor discussed and these assignments were based solely on shell characters.

Only a fraction of the available material was used in the molecular phylogeny of Couto et al. (2016) and we have material of several additional species attributed or attributable to 'Pseudolatirus' as currently recognized that is appropriate for molecular studies. The purpose of the present paper, based on a phylogenetic analysis of an extended molecular dataset of the Fusininae, is to critically re-assess the position of the Recent species that were assigned to Pseudolatirus. Molecular phylogeny combined with morphological studies lead to the recognition of previously unrecognized lineages of Fusininae; which are formally described herein.

\section{Material and methods}

Our study is based on considerably more material than was available to workers before us, and it comes essentially from two sources: material from deep-water oceanographic expeditions in the IndoWest Pacific over the last 40 years or so $(\mathrm{MNHN})$, and material originating from tangle nets and other commercial operations in the Philippines (ex M.A. Snyder' S Collection, now in ANSP). Background information on the expeditions, with narratives of the cruises, station lists, maps, etc. can be found in Richer de Forges $(1990,1991,1993)$ and Richer de Forges \& Chevillon (1996: New Caledonia cruises), Richer de Forges \& Menou (1993: MUSORSTOM 7 cruise to Wallis and Futuna) and Richer de Forges et al. (1996: MUSORSTOM 8 cruise to Vanuatu, 1999: MUSORSTOM 9 cruise to the Marquesas, 2000a and 2000b: Fiji cruises). As a result of these expeditions, over 800 new species of molluscs have been described from the deep waters off New Caledonia alone (Héros et al. 2016).

Specimens from the general collection of MNHN are mostly uncatalogued. Part of the material was specifically preserved for molecular studies and each of those specimens bears a unique registration number, as do the type specimens. In the text we present registration numbers for every catalogued specimen and mention whether it was sequenced or not.

\section{Taxon sampling}

Material for molecular studies was collected in New Caledonia, the Solomon Islands, Papua New Guinea, Madagascar, Taiwan and Vanuatu in a series of deep water cruises (Table 1). Until 2012, live specimens for molecular analysis were anaesthetized with an isotonic solution of $\mathrm{MgCl}_{2}$ and fixed in $96 \%$ ethanol. Specimens collected during later expeditions were processed with a microwave oven (Galindo et al. 2014): the living molluscs in small volumes of sea water were exposed to microwaves for 7-30 seconds, depending on specimen size. Bodies were immediately removed from shells and placed in 96\% ethanol. Specimens were registered in the MNHN collection and sequences were deposited in BOLD (Barcode of Life Datasystem) and GenBank (Table 1). The GenBank accession numbers are provided for corresponding genes in the last three columns in Table 1.

Radulae were prepared by standard methods (Kantor \& Puillandre 2012) and examined with scanning electron microscope TeScan TS5130MM at the Institute of Ecology and Evolution of Russian Academy of Sciences (IEE RAS).

\section{DNA extraction and PCR}

Total genomic DNA was extracted from foot muscle tissue using either NucleoSpinR 96 Tissues (Macherey-Nagel) reagent kit, or Thermo Scientific GeneJET Genomic DNA Purification 50 Kit following the manufacturer's instructions. Fragments of the mitochondrial gene Cytochrome Oxidase I (COI) as well as of the nuclear genes $28 \mathrm{~S}$ rRNA and $\mathrm{H} 3$ were amplified. Standard pairs of primers: LCO1490 and HCO2198 (Folmer et al. 1994) for COI, C1 and D2 (Jovelin \& Justine 2001) for 28S 


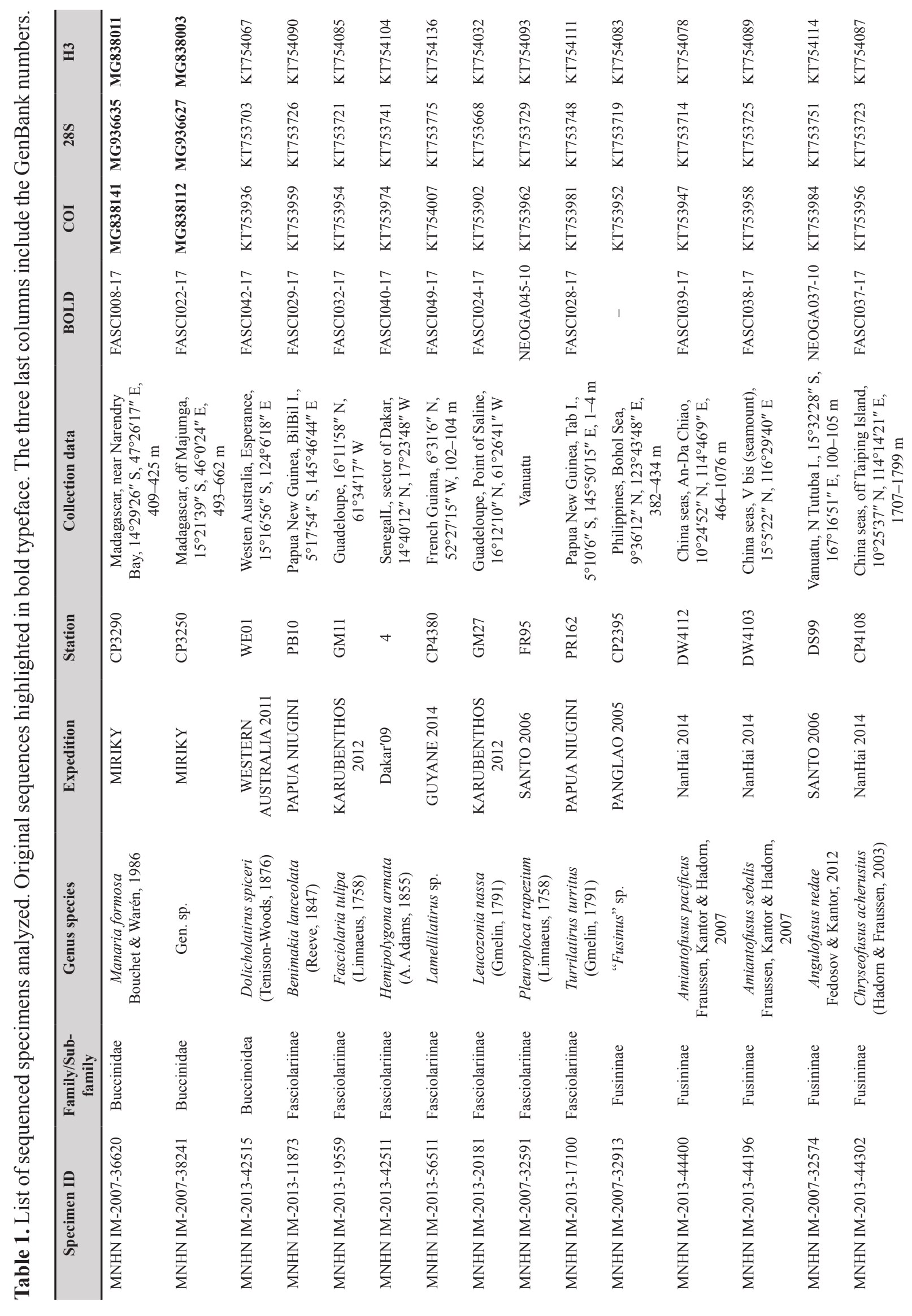




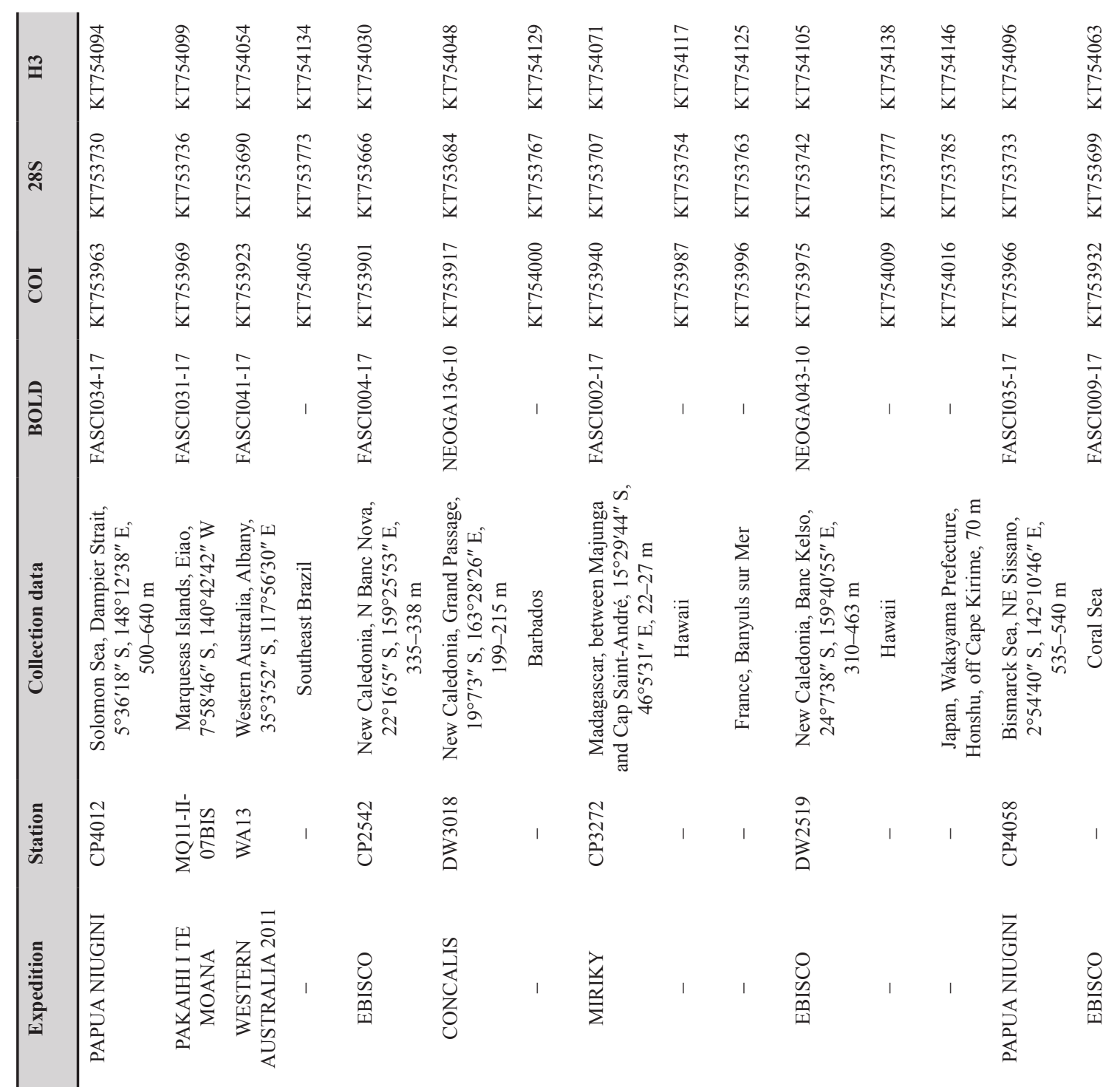

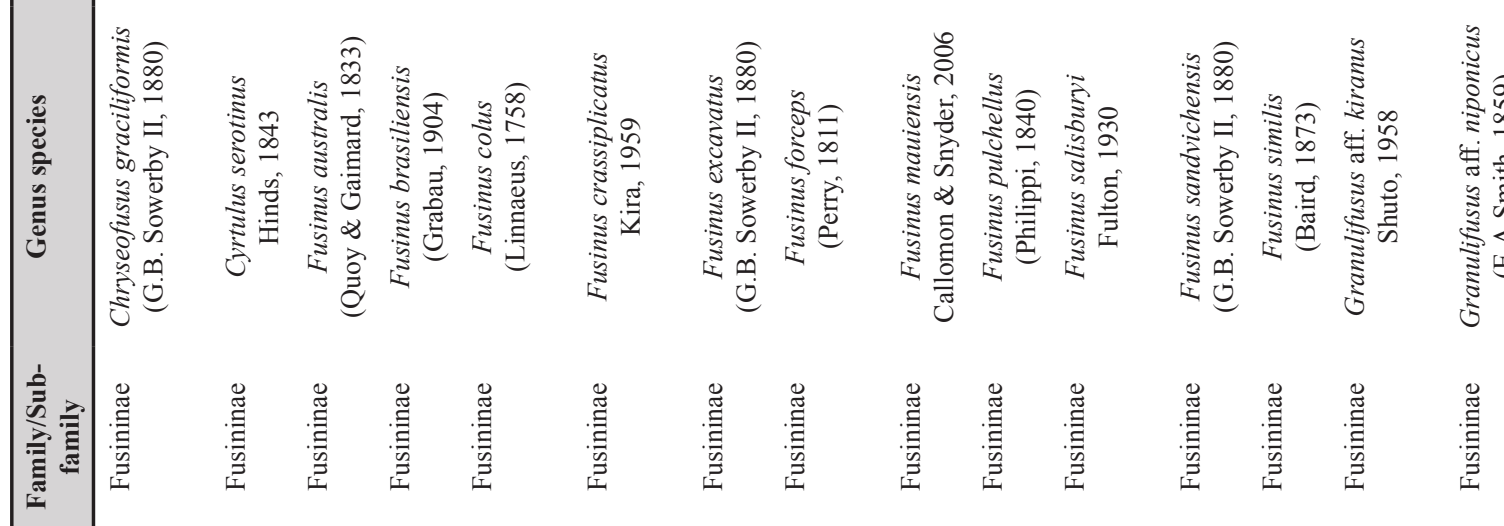

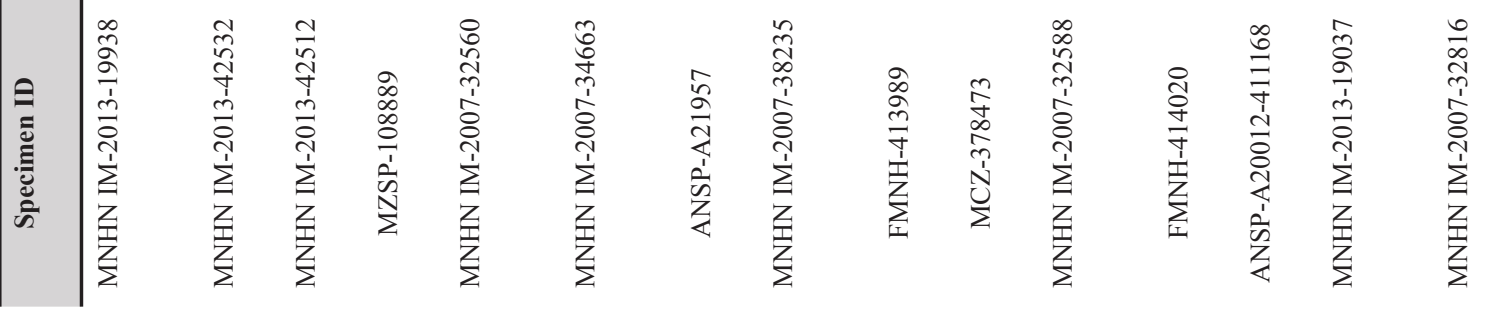




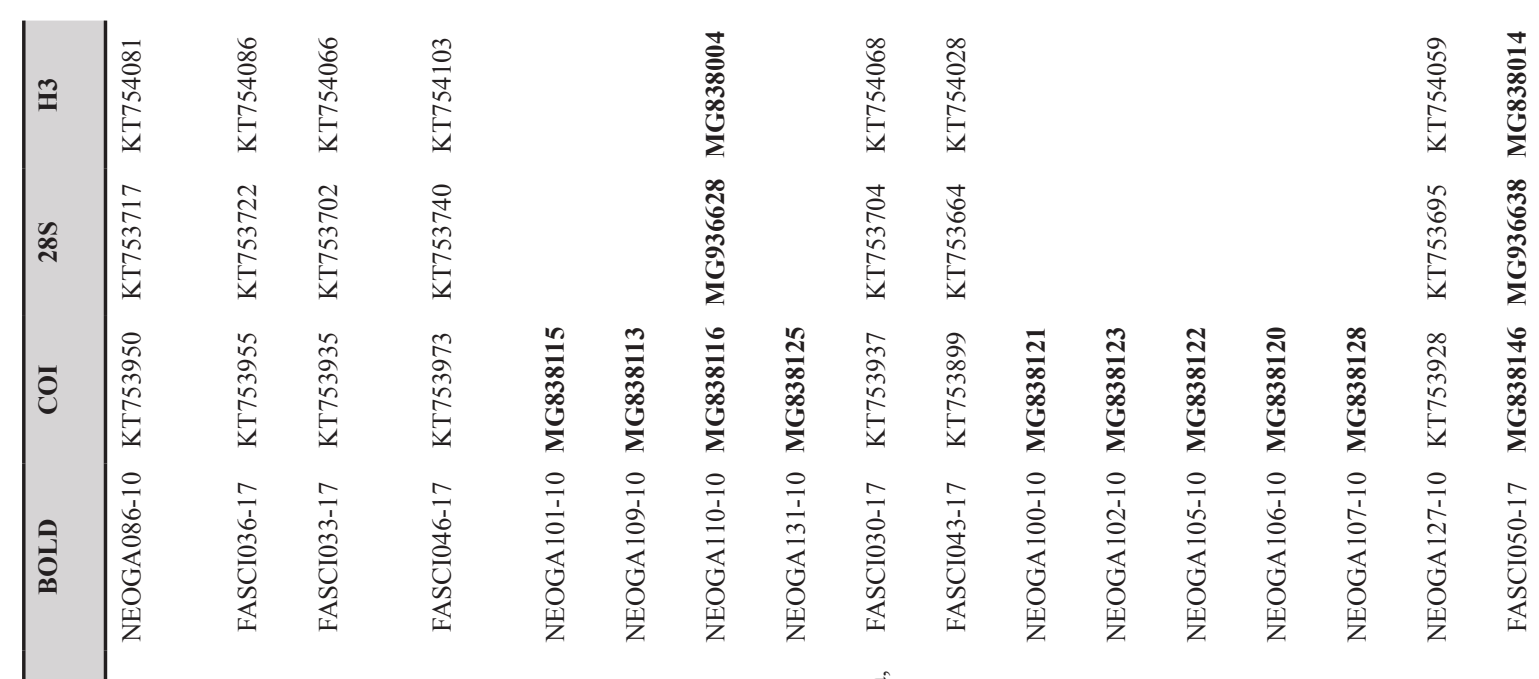

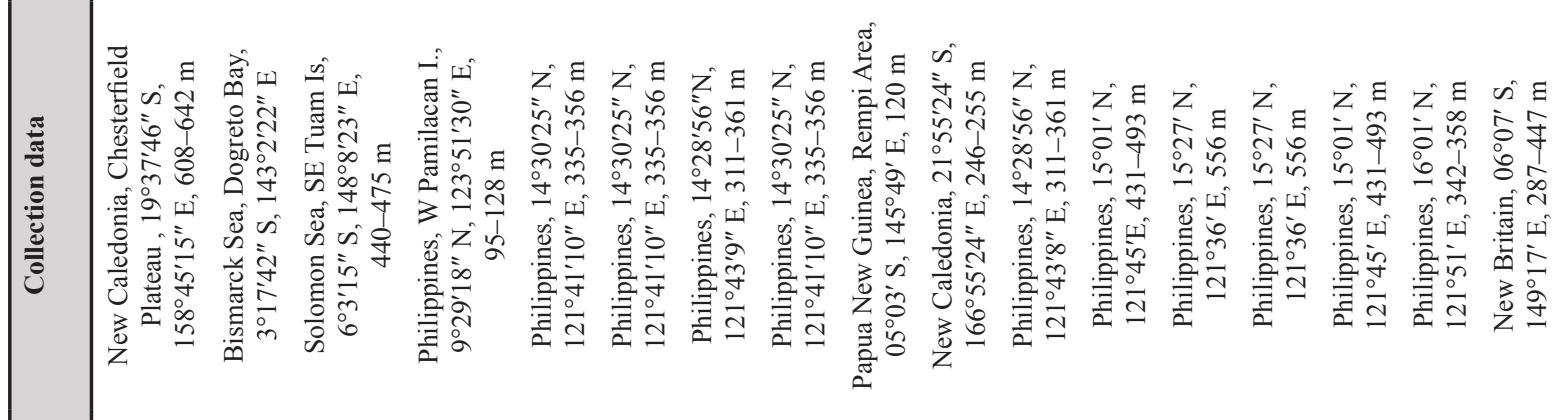

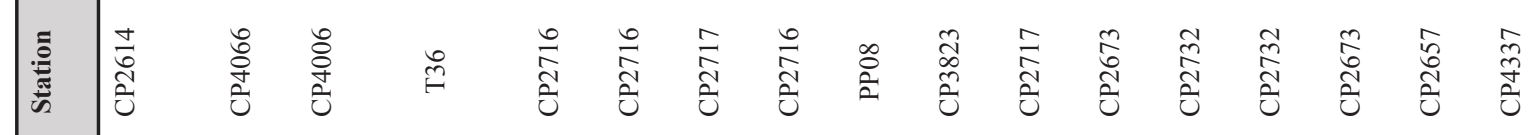

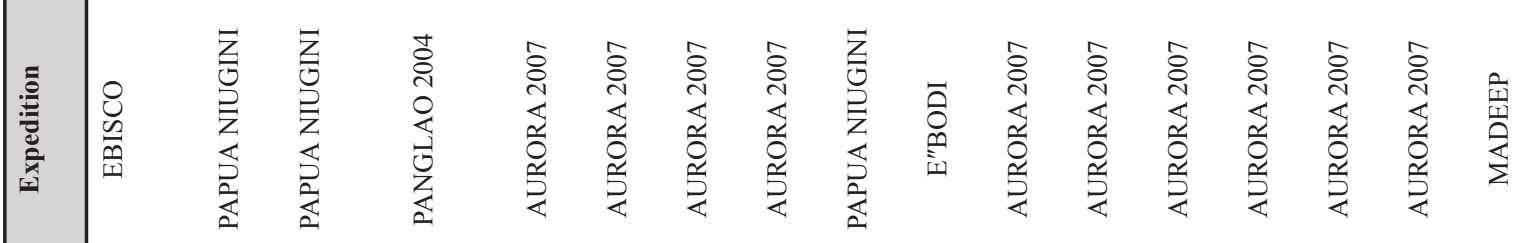

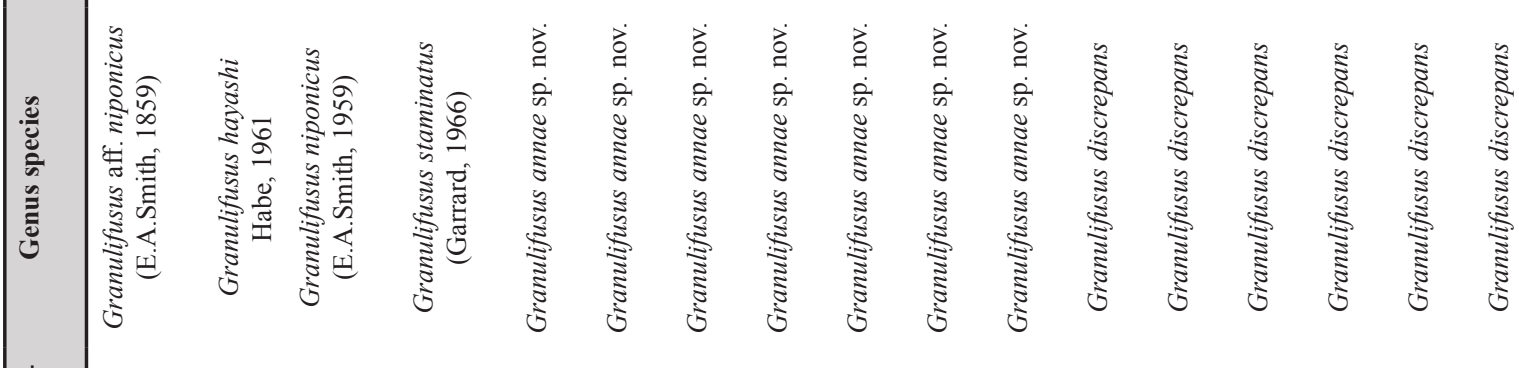

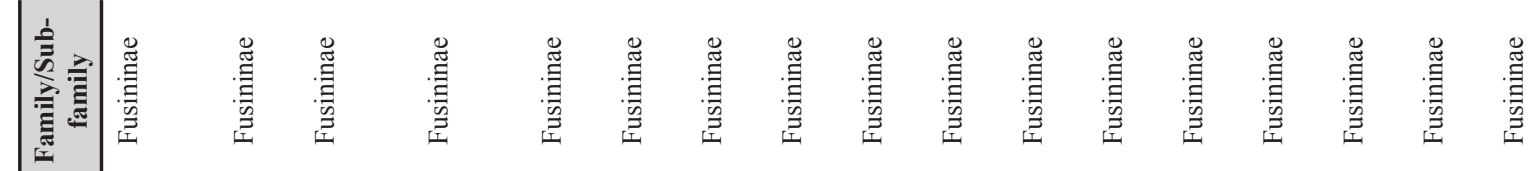

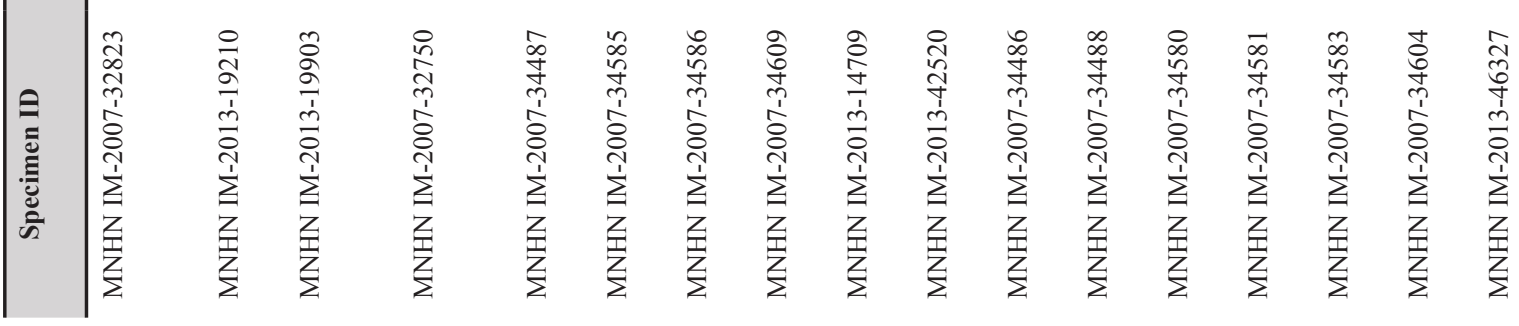




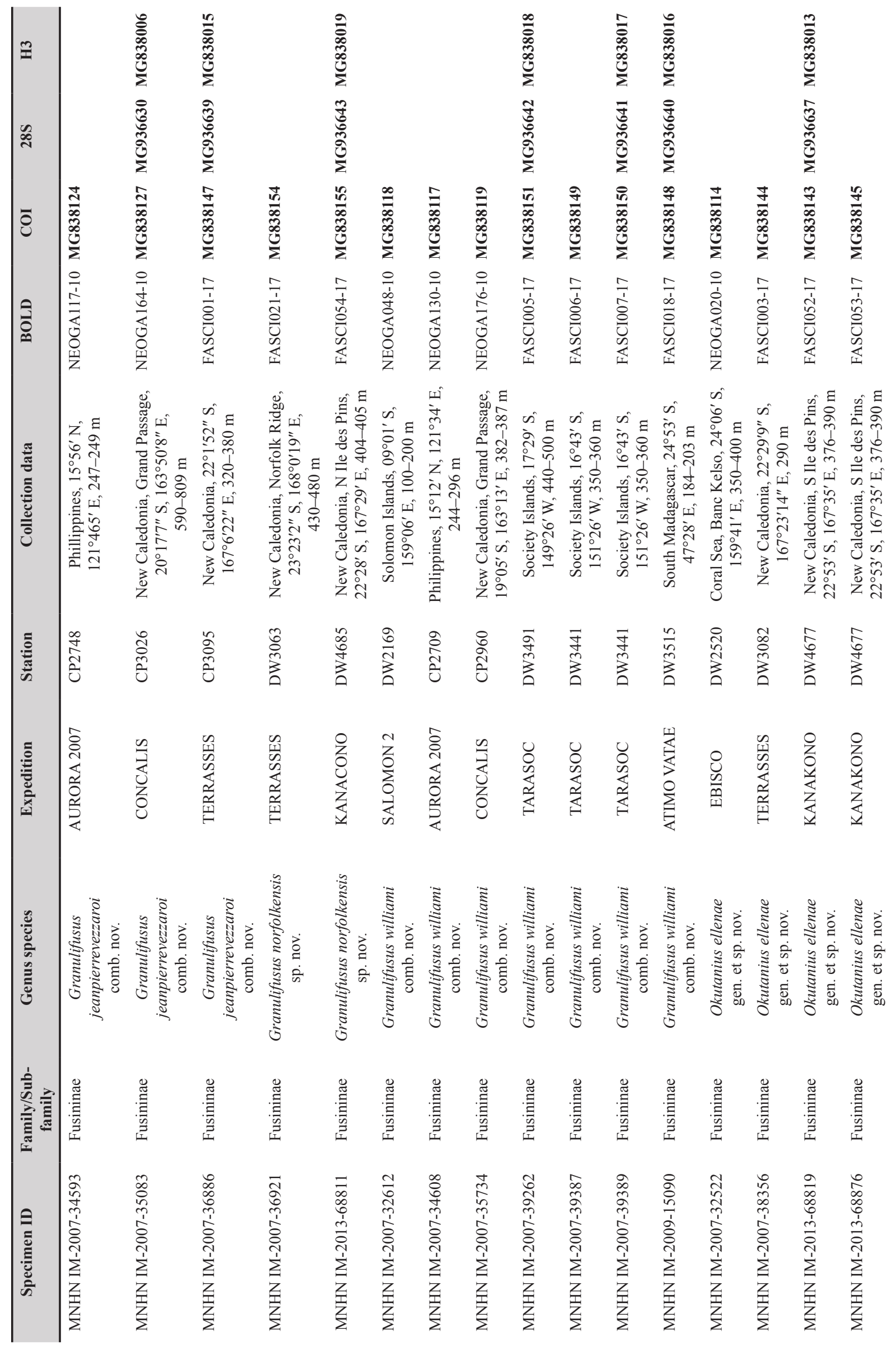




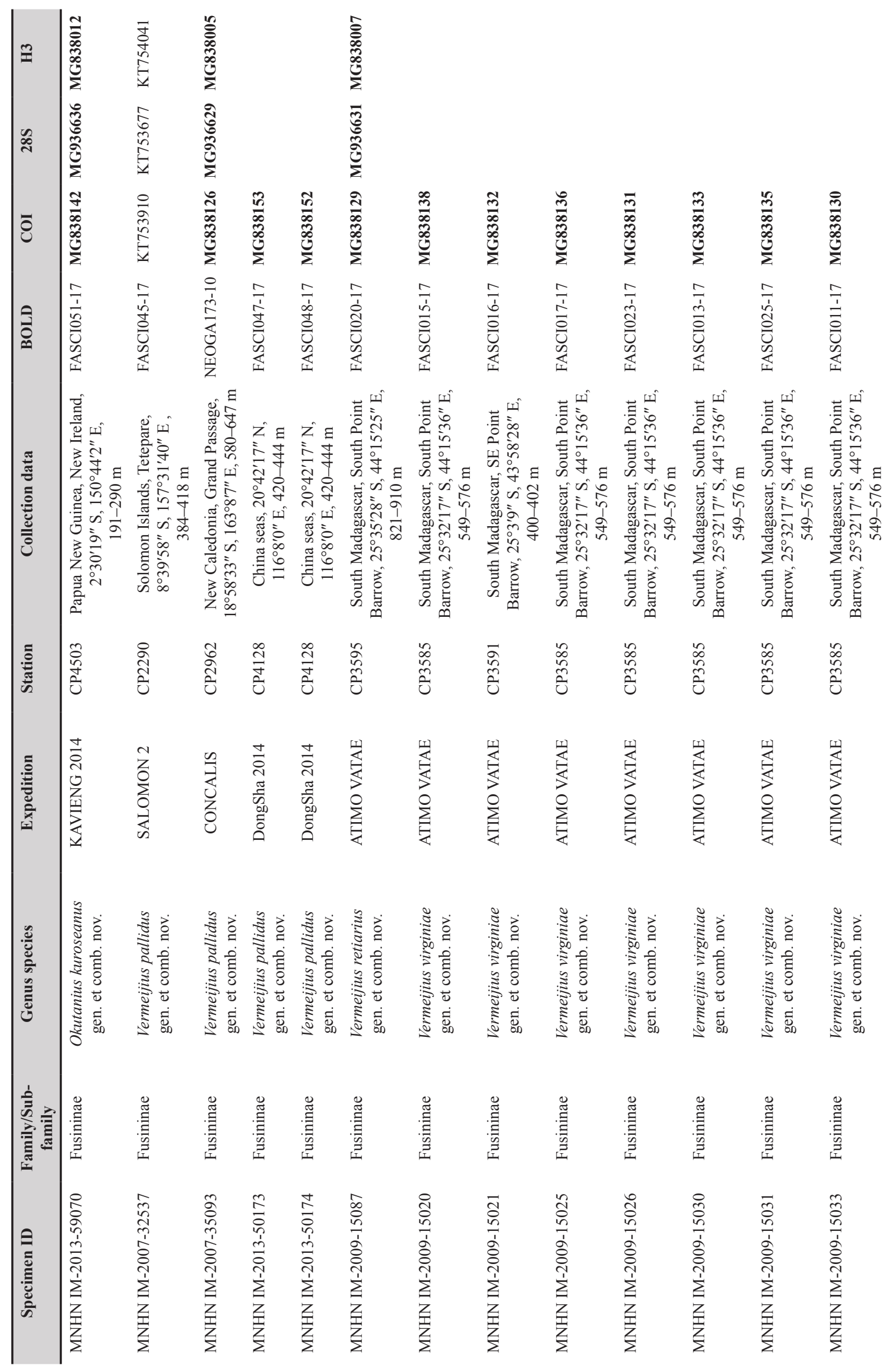




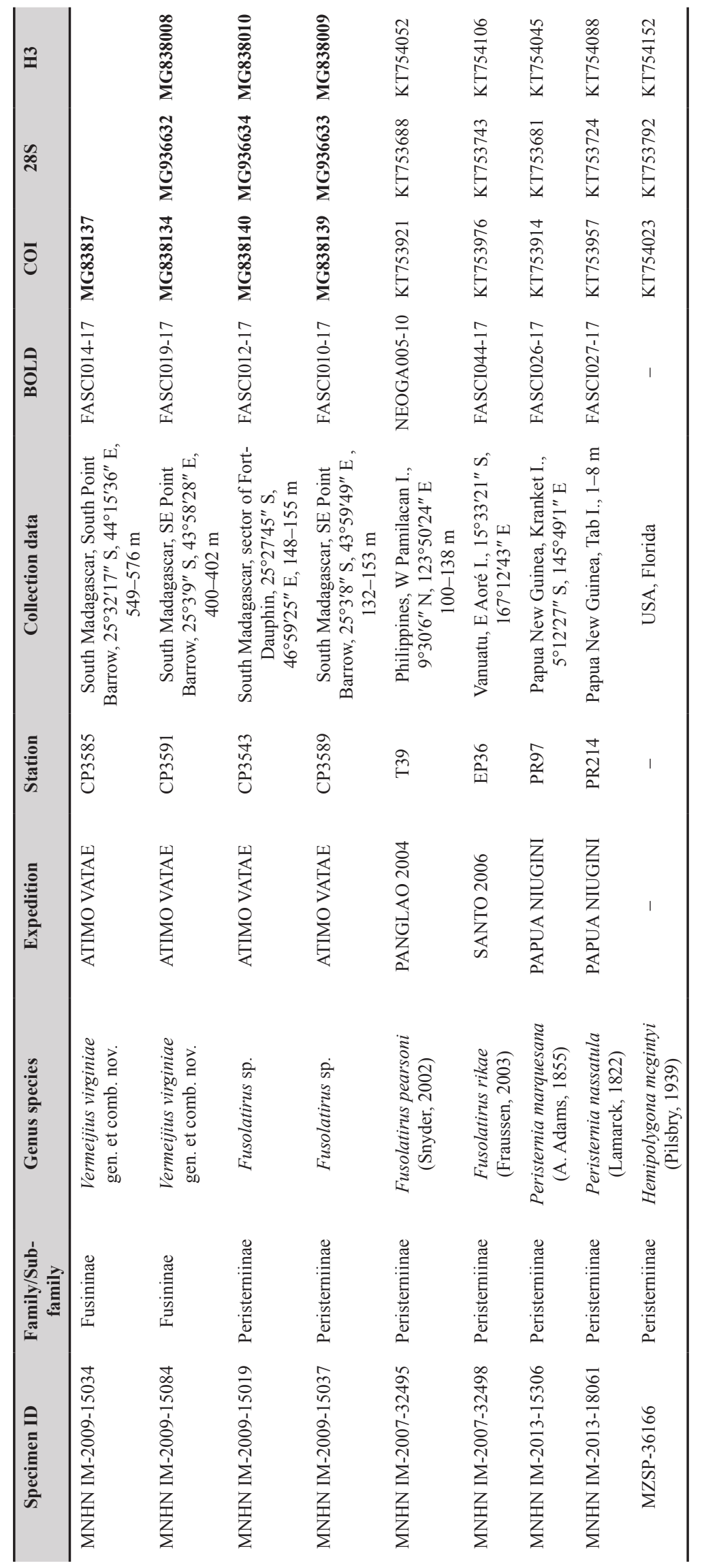


rRNA, and H3F and H3R (Colgan et al. 2000) for H3 were used for polymerase chain reactions (PCR). PCRs were performed in $20 \mu \mathrm{l}$ final volume containing approximately $3 \mathrm{ng}$ template DNA, $1.5 \mathrm{mM}$ $\mathrm{MgCl} 2,0.26 \mathrm{mM}$ of each nucleotide, $0.3 \mu \mathrm{l}$ of each primer, $5 \%$ DMSO and either $0.75 \mu \mathrm{l}$ of Taq Polymerase (Qbiogene) or BioHYTaq DNA polymerase (Dialat).

The PCR profile for the COI started with $5 \mathrm{~min}$ at $95^{\circ} \mathrm{C}$ followed by 40 cycles with the denaturation at $95^{\circ} \mathrm{C}(35 \mathrm{sec})$, annealing at $50^{\circ} \mathrm{C}(35 \mathrm{~s})$ and elongation at $72^{\circ} \mathrm{C}(1 \mathrm{~min})$, with final elongation phase at $72^{\circ} \mathrm{C}\left(10 \mathrm{~min}\right.$ ). Similar PCR profiles were set for $28 \mathrm{~S}$ (annealing at $56^{\circ} \mathrm{C}$ ), and $\mathrm{H} 3$ (annealing at $57^{\circ} \mathrm{C}$, elongation at $72^{\circ} \mathrm{C}$ for $45 \mathrm{~s}$ ). All genes were sequenced in both directions to confirm accuracy of each sequence. The sequencing was performed by Eurofins or in the SIEE RAS molecular facility on an ABI 3500 Genetic analyser.

\section{Phylogenetic analysis}

Sequences were aligned for each gene independently using Clustal $\mathrm{W}$, as implemented in BioEdit v. 7.0.9.0 (Hall 1999). The accuracy of automatic alignments was confirmed by eye. No indels were detected in protein coding genes $\mathrm{COI}$ and $\mathrm{H} 3$, the fragments $658 \mathrm{bp}$ and $328 \mathrm{bp}$ in length were recovered for these two markers respectively; few indels were identified in the $28 \mathrm{~S}$ fragment leading to the alignment of 700 bp in length.

For phylogenetic inference, four datasets were analyzed. Three of them corresponded to the three individual genes, with the COI dataset including 71 sequencies, and $\mathrm{H} 3$ and $28 \mathrm{~S}$ datasets including 57 sequences each, and were analyzed separately. The resulting single gene trees were then checked for consistency (i.e., absence of strongly supported conflicting topologies), and a concatenated dataset of the three genes and $1687 \mathrm{bp}$ was then constructed.

A combination of a distance based approach ABGD (Puillandre et al. 2012), and a tree based approach was used to define molecular taxonomic units (MOTUs) in the analysis of COI, and to compare them with the morphology-based species hypotheses. The maximum intraspecific genetic distance of $3.5 \%$ and a gap width of $1.0 \%$ were set for the ABGD analysis. We checked whether each of the primary species hypotheses corresponded to a monophyletic lineage on the COI based tree and, where there was disagreement, we re-examined voucher shells to check for possible cross-contamination. In this manner the secondary species hypotheses have been refined.

A total of 71 specimens were included in the COI dataset; two deep water Buccinidae (MNHN IM-200736620, Manaria formosa Bouchet \& Warén, 1986 and MNHN IM-2007-38241, Gen. sp.) were used as outgroups (but are not treated in this paper - see Results). The 40 putative Fusininae specimens were included in the multi-gene dataset, together with 7 species from the 'Fasiolaria tulipa (Linnaeus, 1758) clade' (subfamily Fasciolariinae) and 7 species from the 'Peristernia nassatula (Lamarck, 1822) clade' (subfamily Peristerniinae) of Couto et al. (2016). The specimen Dolicholatirus spiceri (Tenison-Wood, 1876) MNHN IM-2013-42515 as well as above mentioned Buccinidae were used as a non-fasciolariid outgroup based on the relationships inferred by Couto et al. (2016).

The phylogenetic relationships among Fusininae lineages were estimated by Bayesian inference (Huelsenbeck \& Ronquist 2001). Bayesian Analyses (BAs) were performed running two parallel analyses in MrBayes with parameters of substitution models estimated during the run. Three partitions corresponding to each codon position were analyzed for the COI and $\mathrm{H} 3$ datasets, and seven partitions that corresponded to the three codon positions of COI, 28S, together with three codon positions of $\mathrm{H} 3$ were analyzed for the concatenated dataset. For primary single-gene analyses, each run consisted of six Markov chains and 5000000 generations with default number of swaps and sampling frequency of 1 tree in each 500 generations. 25000000 generations of Markov chain, and a sampling frequency of one 
tree in each 2000 generations were used in final analyses of COI and COI-28S-H3 datasets. The chain temperature was set at 0.02 in all analyses. Convergence of each analysis was evaluated using Tracer v. 1.6 (Rambaut et al. 2014), where ESS (Effective Sampling Size) values were checked to ensure that they exceeded the threshold value of 200 . Consensus trees were calculated after omitting the first $25 \%$ trees as burn-in.

The same partitions BA were used in the RaxML analysis (Stamatakis 2006), which was performed for the concatenated dataset only. Robustness of nodes was assessed using the Thorough Bootstrapping algorithm (Felsenstein 1985) with 1000 iterations. All analyses were performed on the Cipres Science Gateway (http://www.phylo.org/portal2), using MrBayes v. 3.2.6 on XSEDE (Miller et al. 2010).

To facilitate species identification, COI based molecular diagnoses have been compiled for all Pseudolatirus-like species for which 3 or more COI sequences were available. To draw molecular diagnoses, 658 nucleotide positions in the COI alignment that was used for the phylogenetic reconstructions, were numbered from 1 to 658. Then an in-house Python script (Fedosov et al. unpublished) was run on this alignment, to identify diagnostic combinations of nucleotides for each predefined species. The diagnostic nucleotide combinations, therefore generally correspond to the compound Character Attributes as implemented in CAOS (Sarkar et al. 2008). In order to increase robustness of the diagnosis, for each species we report only independent diagnostic combinations - i.e., a nucleotide position once included in one diagnostic combination will not be involved in any other diagnostic combination.

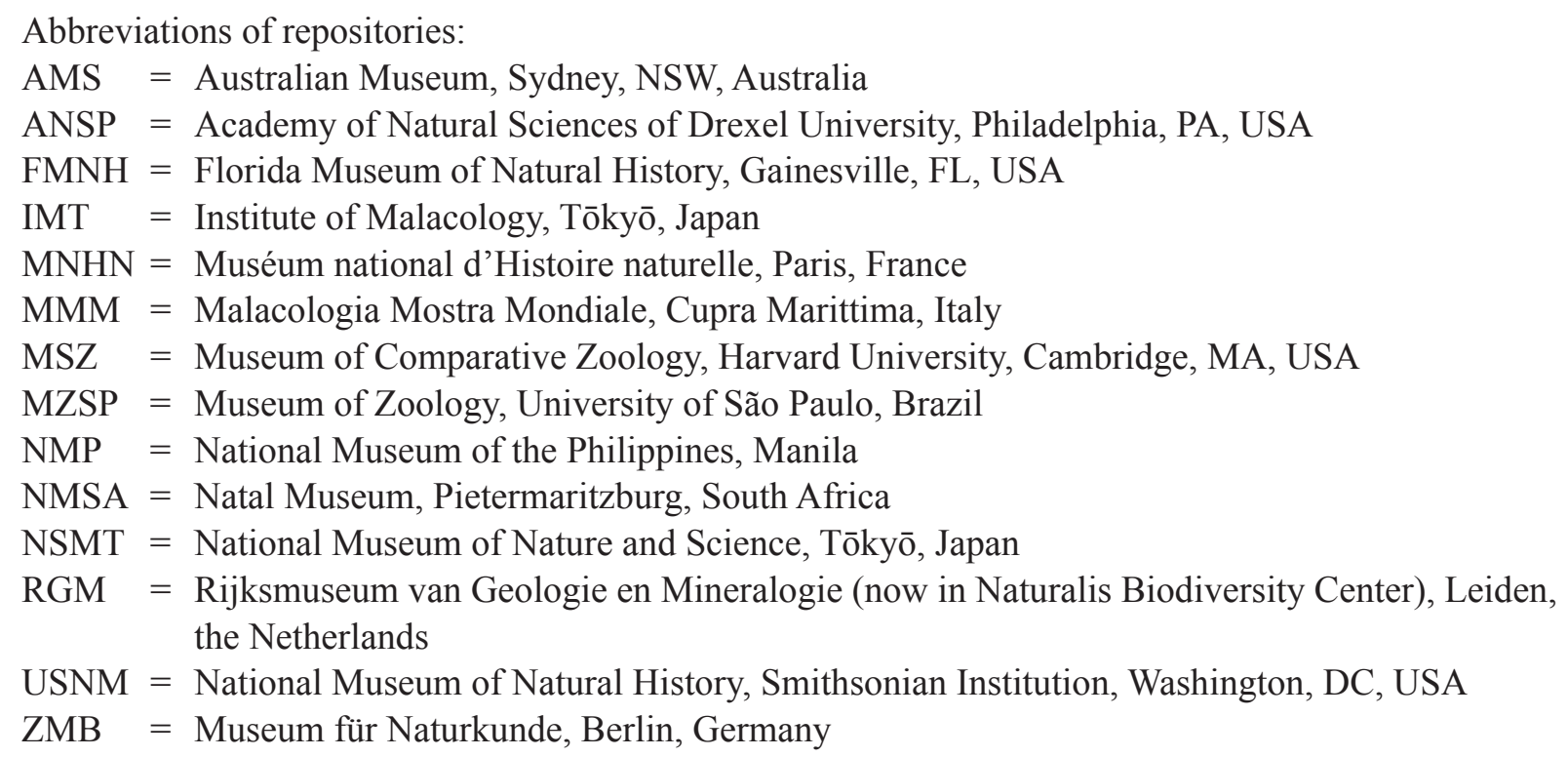

Other text abbreviations:

$\mathrm{AL}=$ aperture length

dd $\quad=$ empty shell

juv $\quad=$ juvenile

$\mathrm{lv} \quad=$ live-collected specimen

MOTU $=$ molecular operational taxonomic unit

$\mathrm{SL} \quad=$ shell length

spm $=$ specimen $(\mathrm{dd}$ and/or $\mathrm{lv})$

stn $=$ station

$\mathrm{SW} \quad=$ shell width 


\section{Results}

\section{Species delimitation and phylogenetic analysis}

Shell morphology led to the identification of ten primary species hypotheses among Pseudolatirus-like species available for sequencing. The COI tree (Fig. 1) is characterized by high support of the terminal clusters (MOTU 1-10), but deeper nodes often lack support. Nine species hypotheses correspond to nine reciprocally monophyletic clades with high nodal support (Bayesian posterior probabilities 0.991). The COI dataset for Fusininae, once analysed with ABGD was split into 29 partitions, of which 10 corresponded to the Pseudolatirus-like specimens. The genetically divergent specimen MNHN IM-2007-32612 (MOTU2) was split in the partition of its own. Nevertheless morphologically this specimens falls within the intraspecific variability of the species. At the same time the MOTUs 6 and 7 were placed in one partition by the ABGD. Contrary to previous case, these MOTUs differ markedly in shell morphology precluding uniting them into the single species. Based on the obtained phylogenetic tree, we have critically revisited species hypotheses, and define species consistent with the proposed MOTUs, rather than based on the partitions proposed by the ABGD.

On the basis of comparison with the type material four MOTUs were identified as species previously described in, or assigned to, Pseudolatirus: MOTU4 as Pseudolatirus jeanpierrevezzaroi Cossignani, 2017; MOTU5 as Pseudolatirus discrepans Kuroda \& Habe, 1961; MOTU7 as Fusolatirus kuroseanus Okutani, 1975; MOTU9 as Pseudolatirus pallidus Kuroda \& Habe, 1961 (these are labelled in red on Figs 1-2). Additionally MOTU2 was identified as Fusinus williami Poppe \& Tagaro, 2006; MOTU8 as Fusinus virginiae Hadorn \& Fraussen, 2002. MOTU10 identified as Fusus retiarius von Martens, 1901 was represented in our material by a single specimen (MNHN IM-2009-15087). Three MOTUs (1, 3, and 6) were recognized as new species and are described herein.

The species previously assigned to Pseudolatirus do not constitute a monophyletic group, and are scattered across the tree. Pseudolatirus pallidus is recovered as a sister species to Fusinus virginiae, albeit with low support, and this two-species clade splits off at the basal polytomy of the COI tree. Fusolatirus kuroseanus forms a highly supported clade with the new species MOTU6 and the resulting clade shows supported affinity to Angulofusus nedae Fedosov \& Kantor, 2012. Pseudolatirus discrepans is recovered as a sister group to several Granulifusus species although without support. Granulifusus in a previously accepted volume (Hadorn \& Fraussen 2005) does not correspond to a monophyletic clade, since Granulifusus staminatus (Garrard, 1966) constitutes a separate phylogenetic lineage.

No supported conflicts were recovered in the single gene analyses, and the analysis of a concatenated dataset returned a tree with notably higher nodal support values (Fig. 2). In this tree, representatives of Fasciolariidae form three distinct lineages. Two of them correspond to the Fasciolaria tulipa and Peristernia nassatula clades identified by Couto et al. (2016) (marked with green and pink respectively). These two clades show a well supported affinity and represent the subfamilies Fasciolariinae and Peristerniinae, respectively. The weakly supported (BI 0.92, bootstrap value 66) Fusininae clade forms a sister group to the Fasciolariinae - Peristerniinae, with high support (BI 1, bootstrap value 94). The minor clade comprising Manaria formosa and an undescribed Buccinidae species from deep water off Madagascar (MNHN IM-2007-38241 and MNHN IM-2007-36620) is recovered as a sister group to all other Fasciolariidae; these species are not dealt with in this paper.

The Fusininae itself clearly splits into four clades, all with high support in Bayesian analysis (BI 0.960.99) albeit lower support in RaxML analysis (bootstrap values 58-76). The first (marked $\mathbf{A}$ on the tree) includes Fusinus s.s. and Amiantofusus; the second (B) combines Chryseofusus Hadorn \& Fraussen, 2003, and three species in a highly supported clade (Fusinus virginiae, Fusus retiarius and Pseudolatirus 


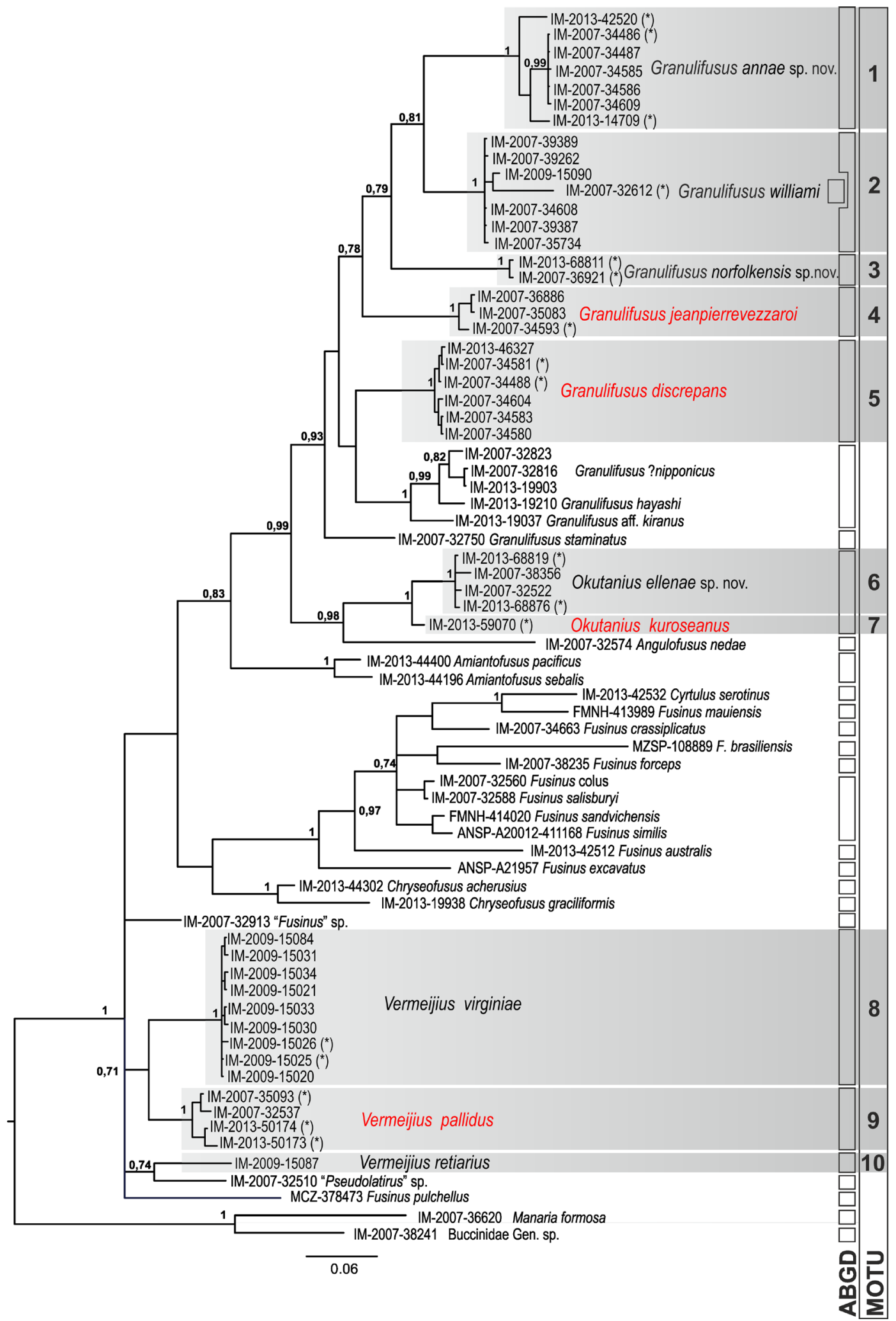

Fig. 1. Bayesian phylogenetic tree of the subfamily Fusininae based on the COI gene. Support values as Bayesian posterior probabilities (PP) indicated when $>0.7$. Asterisks mark specimens illustrated on figures. The names of the species previously attributed to Pseudolatirus are given in orange. Partitions recovered by ABGD marked as vertical line of rectangles on the right. MOTUs identified in the current study are highlighted in grey. 


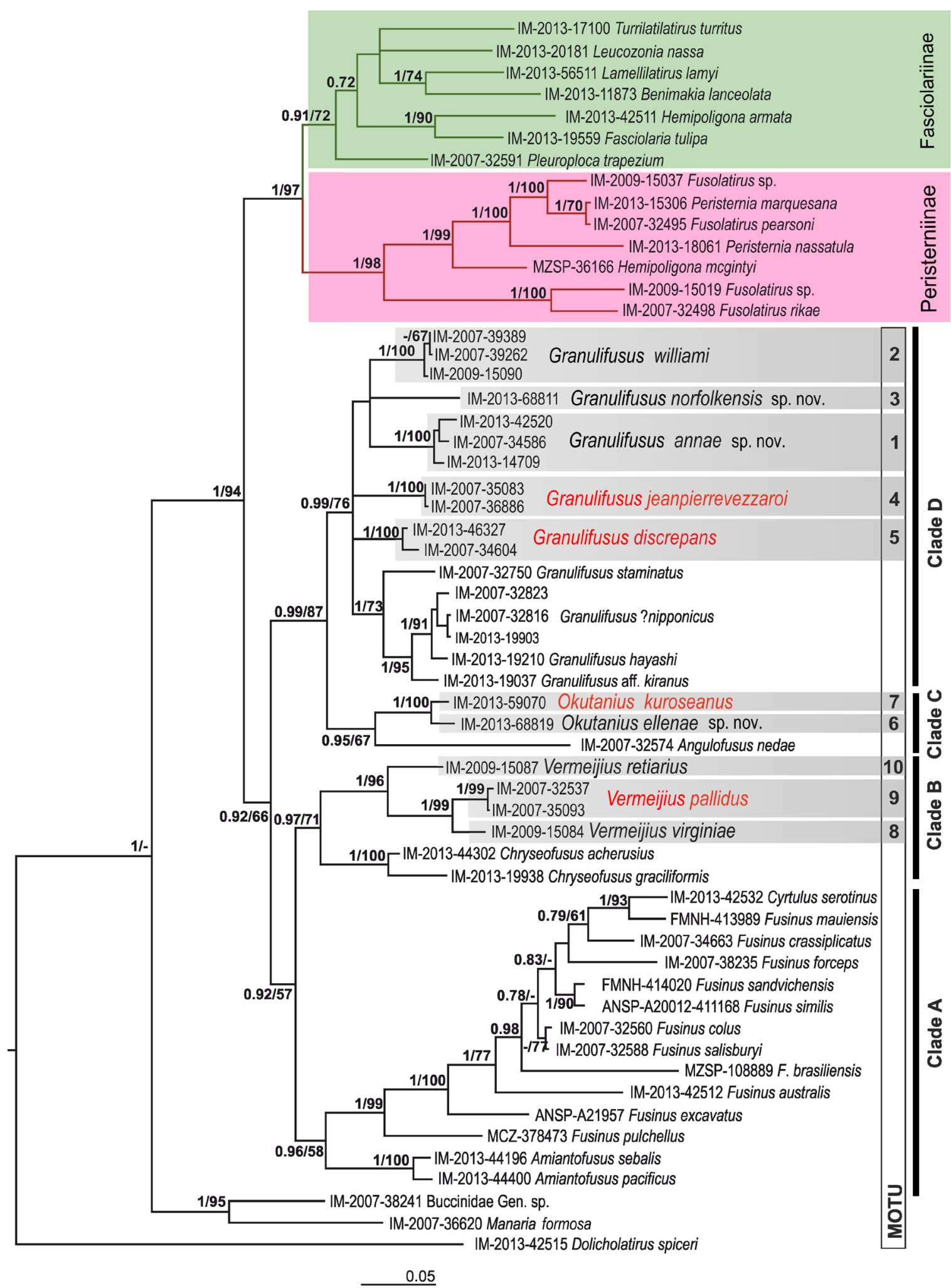

Fig. 2. Bayesian phylogenetic tree of Fasciolariidae based on the combined COI-H3-28S dataset. Subclades corresponding to Couto et al.'s (2016) Fasciolaria tulipa clade (Fasciolariinae) and Peristernia nassatula clade (Peristerniinae) are marked with green and pink, respectively. MOTUs identified in the current study are highlighted in grey. The numbering of MOTU correspond to Fig. 1. Support values as Bayesian posterior probabilities (PP) indicated when $>0.7$ / bootstrap values, when $>50$. 
pallidus). The third clade (C) includes Angulofusus nedae and a highly supported clade that includes two species, Fusolatirus kuroseanus and MOTU6.

In the fourth clade (D), a well-supported in Bayesian analysis crown group combines species attributed presently to Granulifusus and five maximal supported single-species clades, of which three correspond to Pseudolatirus discrepans, Pseudolatirus jeanpierrevezzaroi and Fusinus williami and two others, MOTUs 1 and 3. These five species are rather heterogeneous in morphology, although some common characters can be enumerated: a strong shell with a well-attenuated narrow canal, rather strong axial sculpture of few ribs, presence of weak to moderately developed columellar plicae in most specimens, and a radula with narrow central tooth.

\section{Taxonomic account}

An important taxonomic question related to the group, is whether the genus name Pseudolatirus can be attributed to any of the newly defined clades, which includes the species previously assigned to, or described as, Pseudolatirus. In fact, the Recent species of 'Pseudolatirus' bear little resemblance to the fossil true Pseudolatirus. Only the young specimens of 'Pseudolatirus' discrepans have conchological similarities with the type species of Pseudolatirus, Fusus bilineatus, notably in the spiral and axial sculpture and the presence of a weak columellar fold delimiting the siphonal canal (Fig. 3). There is a significant ontogenic change in the teleoconch sculpture pattern in 'Pseudolatirus' discrepans, with earlier whorls bearing strong axial ribs while later whorls in large shells can be nearly smooth. However, a sculpture pattern similar to that in F. bilineatus is found throughout the shells grouped in Peristerniinae (to which we also attribute Pseudolatirus), and is insufficient to establish relationships at the generic level. Since F. bilineatus is a fossil species from the Miocene, the connections to Recent material have to be especially convincing if the Recent material is to be placed in a genus with a fossil type species, and in the present case the evidence is not sufficiently strong. For this reason we abstain from using the name Pseudolatirus for any Recent species, that necessitates to reaccess the generic position of the species formerly assigned to Pseudolatirus.

Therefore we erect Vermeijius gen. nov. (type species Pseudolatirus pallidus) for the subclade in clade B uniting MOTUs 8-10, Okutanius gen. nov. (type species Fusolatirus kuroseanus) for F. kuroseanus and MOTU 6 (described herein as a new species, $O$. ellenae gen. et sp. nov.) forming a subclade in clade C. While erecting these two new genera is straightforward and is undoubtedly supported by the molecular phylogeny, the status of the five species (MOTUs 1-5) in clade D is less obvious. Species traditionally included in Granulifusus (and forming a separate, highly supported, branch within the crown clade) differ slightly from the abovementioned five species in having lighter and thinner shells with weaker axial sculpture compared to shells of the abovementioned five species, together with spiral cords forming beads or 'granules' at their intersection with the axial ribs. Protoconchs of Granulifusus have up to 1.5 whorls (Hadorn \& Fraussen 2005) vs 2.25 (MOTU1) to 4.25 (Fusinus williami). Most studied species of Granulifusus possess a radula with a shorter and therefore relatively broader central tooth compared to these five species (Hadorn \& Fraussen 2005: figs 109-121). An exception is G. staminatus which has a narrow central tooth, similar to Pseudolatirus-like species of subclade D (see Fig. 4 on page 19). In none of the analyses these five species form a clade, while the clade uniting them and Granulifusus spp. was always highly supported. The internal relationships within the crown clade are not resolved and it is not impossible however that a more saturated molecular dataset will result in the mentioned species forming a monophyletic clade(s). Nevertheless, we consider it premature to establish a new genus for them and based on the results of molecular analyses have to assign Pseudolatirus discrepans, Pseudolatirus jeanpierrevezzaroi, Fusinus williami and MOTUs 1 and 3, described herein as new species to Granulifusus albeit with some reservations. 


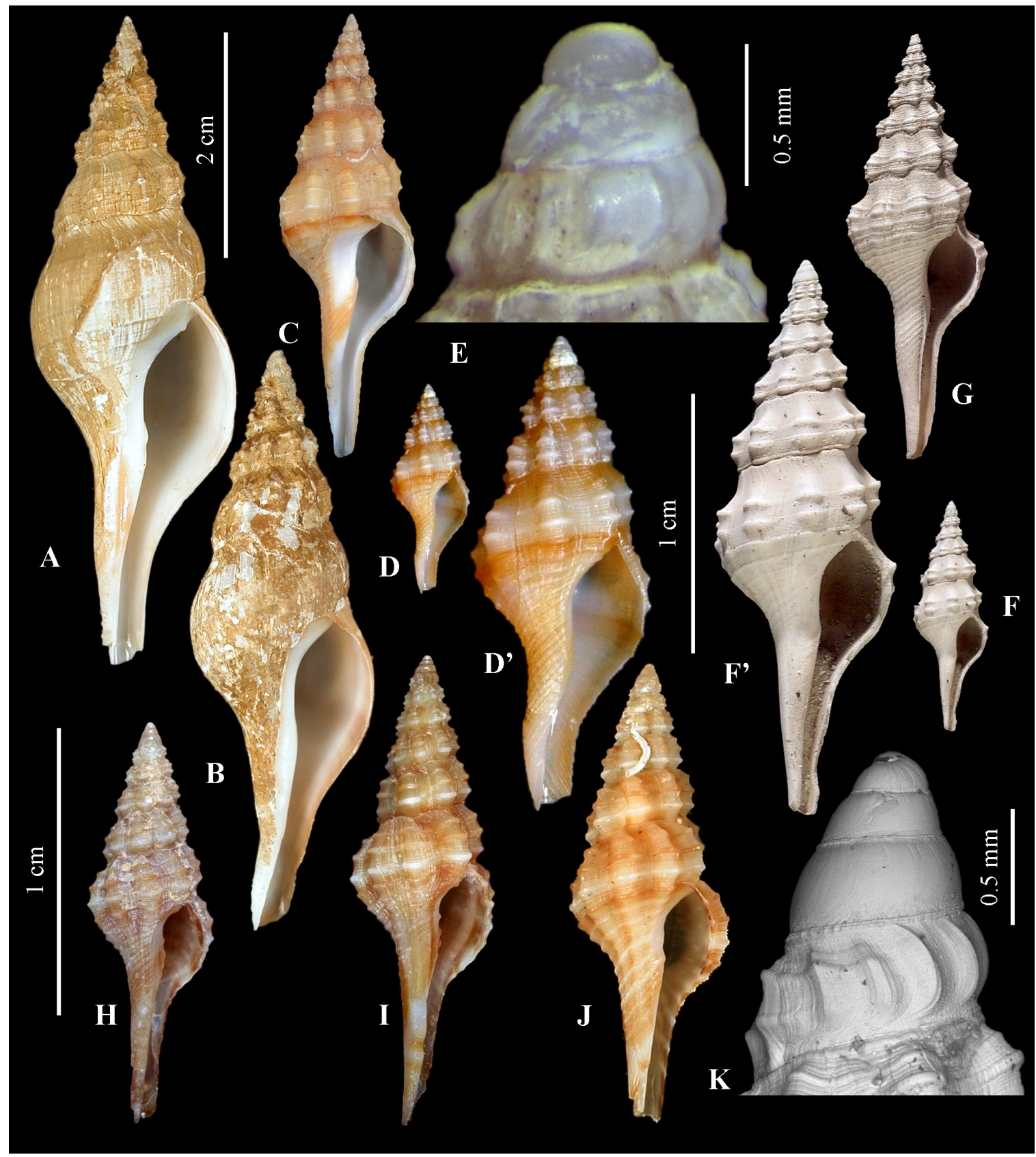

Fig. 3. A-E. Granulifusus discrepans (Kuroda \& Habe, 1961). A. Philippines, SL 58 mm, sequenced specimen (MNHN IM-2007-34581). B. Philippines, SL $51.9 \mathrm{~mm}$, sequenced specimen (MNHN IM2007-34488). C. Papua New Guinea, PAPUA NIUGINI, stn CP3949, 05²12' S, $145^{\circ} 51^{\prime}$ E, 380-407 m (MNHN 2013-15374, not sequenced). D-E. Solomon Sea, SL $18.2 \mathrm{~mm}$, sequenced specimen (MNHN IM-2013-46327). D'. Same as preceding, enlarged. - F-G. Pseudolatirus bilineatus (Hörnes, 1853). F. Lectotype, SL $20.8 \mathrm{~mm}$ (NHMW 1846/0037/0263). F'. Same as preceding, enlarged. G. Paralectotype, SL 38.7 mm (NHMW 1846/0037/0264). - H-K. Granulifusus williami (Poppe \& Tagaro, 2006) comb. nov. H. Papua New Guinea, New Ireland, KAVIENG 2014, stn DW4471, 02 $44^{\prime}$ S, $150^{\circ} 38^{\prime}$ E, $126-$ $220 \mathrm{~m}$, SL $14.1 \mathrm{~mm}$ (MNHN IM-2013-58726, not sequenced). I. Papua New Guinea, New Ireland, KAVIENG 2014, stn DW4484, 02 26' S, 149 55' E, SL 16.5 mm (MNHN IM-2013-58866, not sequenced). J. Solomon Islands, SL $16.1 \mathrm{~mm}$, sequenced specimen (MNHN IM-2007-32612). K. New

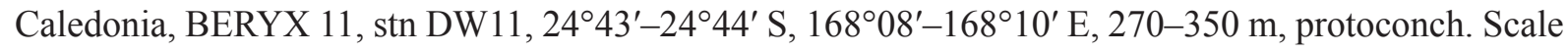
bars: $A-D=2 \mathrm{~cm} ; F-G=1 \mathrm{~cm} ; \mathrm{H}-\mathrm{J}=1 \mathrm{~cm}$. D' and F' to the same scale. 
Four additional Recent species have been assigned (some tentatively) to Pseudolatirus and are not represented in our sequenced material. Fusus clausicaudatus Hinds, 1844 was placed in Pseudolathyrus [= Pseudolatirus] by Kaicher (1986, card no. 4706); it was treated in detail by Stahlschmidt \& Fraussen (2012) who erected the new genus Crassibougia Stahlschmidt \& Fraussen, 2012 to incorporate F. clausicaudatus and one more new species. Pseudolatirus leucostriatus Kosuge, 1979 is herein placed in synonymy with Pseudolatirus kuroseanus. Sabelli et al. (1990: 38) placed Fusus marmoratus Philippi, 1846 in Pseudolatirus, but we consider this species to be a true Fusinus. Finally, Fusus pfeifferi Philippi, 1846, with an uninformative original description and unknown type locality, was tentatively assigned to Pseudolatirus by MacNeil (1961: 83). We hereby treat it as a nomen dubium.

Family Fasciolariidae Gray, 1853

Subfamily Fusininae Wrigley, 1927

Granulifusus Kuroda \& Habe, 1954

\section{Type species}

Fusus niponicus E.A. Smith, 1879, by original designation.

\section{Genus composition}

In addition to species previously assigned to the genus (Hadorn \& Fraussen 2005), we include sequenced species Granulifusus discrepans, G. williami comb. nov., G. jeanpierrevezzaroi comb. nov., G. annae sp. nov. and G. norfolkensis sp. nov. In addition to those species we allocate to Granulifusus, based on conchological similarities to sequenced species, G. tatianae sp. nov., G. kurodai comb. nov., and G. guidoi sp. nov., known from empty shells only.

\section{Granulifusus discrepans (Kuroda \& Habe, 1961)}

Figs $3 \mathrm{~A}-\mathrm{E}, 4 \mathrm{~A}$

Pseudolatirus discrepans Azuma, 1960: 45 (ex Kuroda MS). nomen nudum.

Pseudolatirus discrepans - Kuroda \& Habe in Habe 1961: 66, pl. 33, fig. 6. — Habe 1962: 102, pl. 33, fig. 6. - Higo, Callomon \& Gotō 2001: 87, fig. G3002 (holotype illustrated). — Mallard \& Robin 2017: 152.

Granulifusus discrepans - Poppe 2008: pl. 343, fig. 1.

\section{Molecular diagnosis}

A molecular diagnosis is given in Table 2.

\section{Type material}

\section{Holotype}

JAPAN: off Cape Ashizuri, southwestern Shikoku (NSMT Mo 40377) (not seen).

\section{Sequenced material}

SOLOMON SEA: 1 lv, MADEEP, stn CP4337, SE Ainto Bay, New Britain, 0607’ S, $149^{\circ} 17^{\prime}$ E, $287-$ 447 m (MNHN IM-2013-46327).

PHILIPPINES: AURORA 2007, 1 lv, stn CP2657, 160 $01^{\prime} \mathrm{N}, 121^{\circ} 51^{\prime} \mathrm{E}, 342-358 \mathrm{~m}$ (MNHN IM-200734604); 2 lv, stn CP2673, $15^{\circ} 01^{\prime} \mathrm{N}, 121^{\circ} 45^{\prime} \mathrm{E}, 431-493$ m (MNHN IM-2007-34583, MNHN IM-2007-

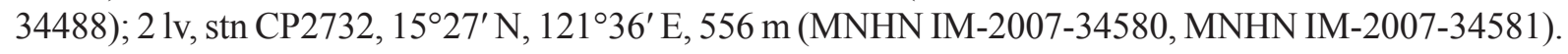


Table 2. Molecular diagnostic characters of species of Vermeijius gen. nov.

\begin{tabular}{|c|c|}
\hline \multicolumn{2}{|c|}{ Granulifusus annae sp. nov. } \\
\hline $\begin{array}{l}\text { Sequences analyzed } \\
\text { Independent combinations: }\end{array}$ & 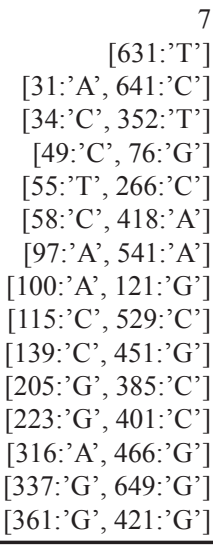 \\
\hline \multicolumn{2}{|c|}{ Granulifusus williami comb. nov. } \\
\hline $\begin{array}{l}\text { Sequences analyzed } \\
\text { Independent combinations }\end{array}$ & 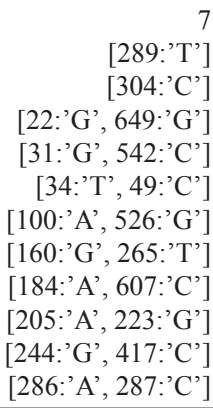 \\
\hline \multicolumn{2}{|c|}{ Granulifusus jeanpierrevezzaroi comb. nov. } \\
\hline $\begin{array}{l}\text { Sequences analyzed } \\
\text { Independent combinations }\end{array}$ & 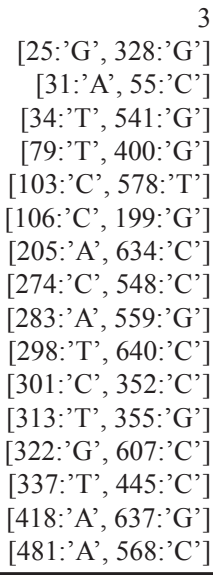 \\
\hline \multicolumn{2}{|c|}{ Granulifusus discrepans (Kuroda \& Habe, 1961) } \\
\hline $\begin{array}{l}\text { Sequences analyzed } \\
\text { Independent combinations }\end{array}$ & 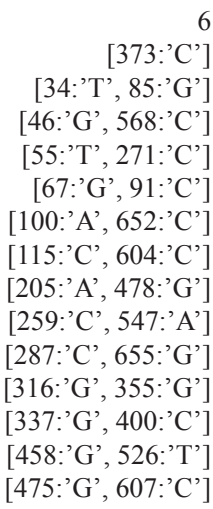 \\
\hline
\end{tabular}



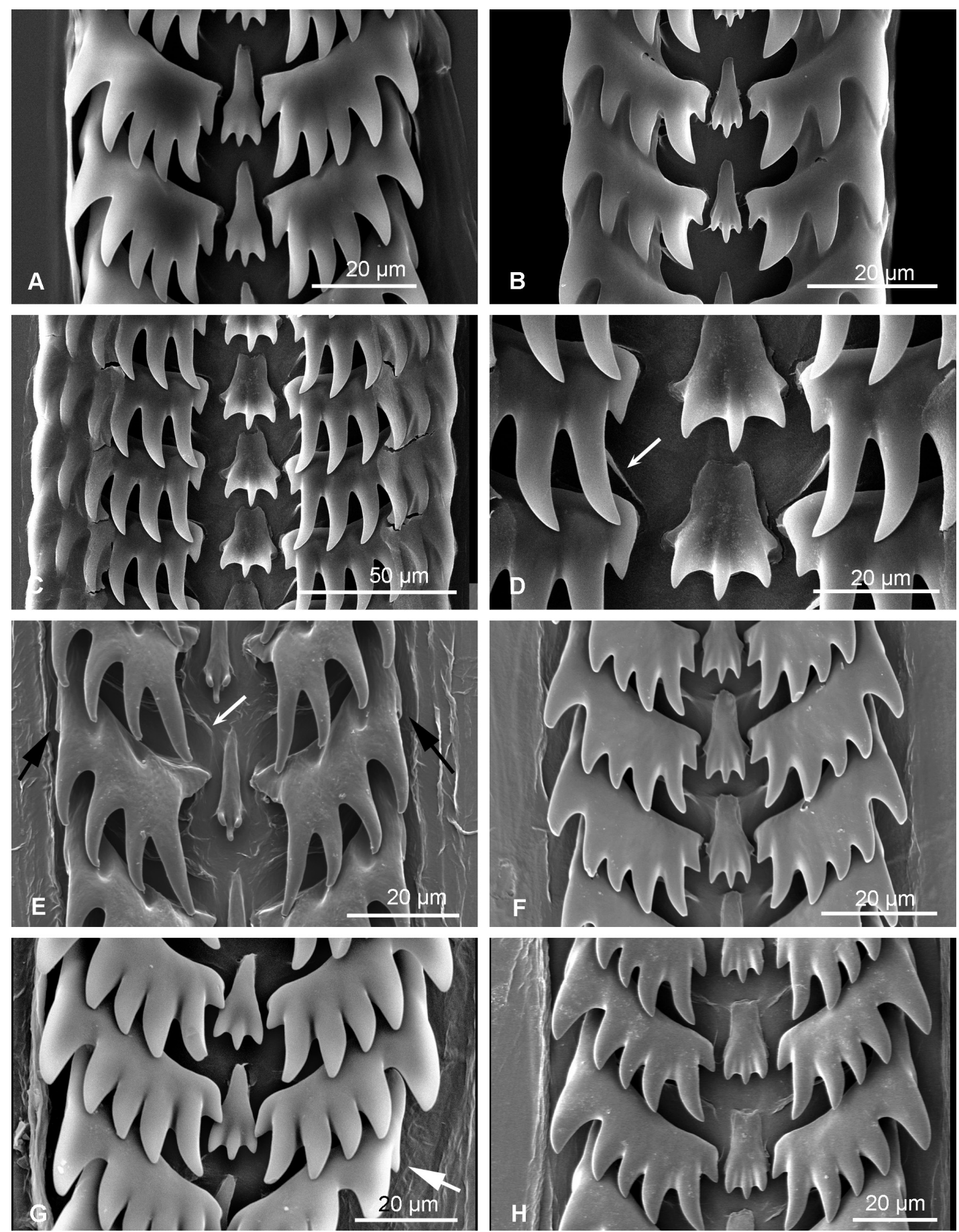

Fig. 4. Radulae of species of Granulifusus and Okutanius gen. nov. A. Granulifusus discrepans (Kuroda \& Habe, 1961), shell, see Fig. 3D (MNHN IM-2013-46327). B. Granulifusus williami (Poppe \& Tagaro, 2006), comb. nov., shell, see Fig. 3H (MNHN IM-2013-58726). C-D. Granulifusus jeanpierrevezzaroi (Cossignani, 2017) comb. nov., shell, see Fig. 5B-D, arrow points to regularly spaced folds of the radular membrane (MNHN IM-2007-34593). E. Granulifusus annae sp. nov., New Caledonia, BATHUS 1, stn CP669, 20 ${ }^{\circ} 57^{\prime}$ S, $165^{\circ} 35^{\prime}$ E, 255-280 m, shell, see Fig. 6F. F. Granulifusus tatianae sp. nov., Loyalty

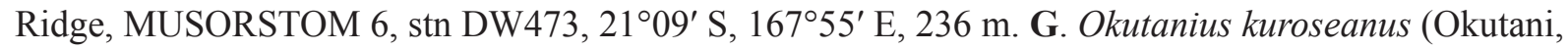
1975) gen. et comb. nov., New Ireland, shell, see Fig. 9A (MNHN IM-2013-59070). H. Okutanius

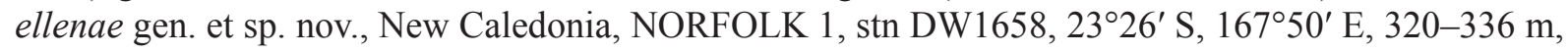
shell, see Fig. 10H). 


\section{Other examined material}

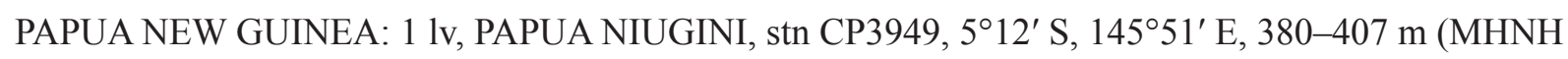
2013-15374, not sequenced); 1 lv, stn CP4023, 5²2' S; 145²4' E, 340-385 m (MNHN IM-2013-9777, not sequenced).

\section{Description}

Shell large, with multispiral protoconch of 2.75 conical, smooth, convex, grayish whorls, last $1 / 4$ whorl with 6-7 rounded axial riblets progressing in strength, protoconch/teleoconch boundary without varix, defined by appearance of spiral sculpture. Protoconch diameter $960 \mu \mathrm{m}$, exposed height $890 \mu \mathrm{m}$ (Fig. 3E). Teleoconch with regularly convex whorls, upper ones slightly angulated, with long slightly sinuous to nearly straight siphonal canal; aperture in young specimens with 3-4 plicae, in adult with a single columellar plica, delimitating siphonal canal, and sometimes with indistinct parietal tooth. Axial sculpture of distinct axial ribs, 7-8 per whorl on upper whorls, obsolete on posterior whorls in shells over $50 \mathrm{~mm}$. Spiral sculpture of 2-3 raised cords on periphery of upper whorls and numerous thin riblets of even strength. On posterior whorls, cords obsolete and some riblets more pronounced. Background colour tan, with darker brown spiral bands along suture.

Radula (MNHN IM-2013-46327, SL $18.2 \mathrm{~mm}$, Fig. 4A) $1.83 \mathrm{~mm}$ in length (32\% of AL without canal), of 75 transverse rows of teeth, of which 35 nascent. Radula narrow, distance between outer edges of lateral teeth about $65 \mu \mathrm{m}(1.12 \%$ of $\mathrm{AL})$. Lateral teeth medium broad, arcuate, with attenuated outer anterior corner, producing short 'handle'. Lateral teeth with five closely spaced, slightly curved cusps similar in length, small outermost cusp (seen on lower right lateral tooth on Fig. 4A), and much smaller but distinct innermost cusp. Central tooth small, very narrow, trapezoid, with three subequal cusps. Narrow but distinct transverse folds of radular membrane situated serially between rows of lateral teeth. Couto (2016: fig. 58) illustrated the radula of an adult specimen (SL $66 \mathrm{~mm}$ ). The lateral teeth were relatively much broader, with 6 main cusps nearly identical in size and an additional innermost cusp. The radula had a similar width ( $1.1 \%$ of AL, measurements taken from photo) to the one studied herein; the differences in teeth morphology can possibly be explained by ontogenetic variability.

\section{Distribution}

From Japan throughout the East China Sea to the Philippines and the Solomons.

\section{Remarks}

This is the largest species of Granulifusus, which attains a shell length over $90 \mathrm{~mm}$ (http://www.conchology.be, last accessed 6 Mar. 2017). Due to the ontogenic change in sculpture pattern, the shell morphology differs from that in other Recent members of the genus, but the radula is similar. Young specimens bear a superficial resemblance to specimens of the Miocene species Pseudolatirus bilineatus of similar size (compare Fig. 3C, G with Fig. 3D', F').

Granulifusus williami (Poppe \& Tagaro, 2006) comb. nov. Figs $3 \mathrm{H}-\mathrm{K}$, 4B

Fusinus williami Poppe \& Tagaro, 2006: 48, plate 1.

Fusinus williami - Mallard \& Robin 2017: 106.

\section{Molecular diagnosis}

A molecular diagnosis is given in Table 2. 


\section{Type material}

\section{Holotype}

PHILIPPINES: off Dipolog, Aliguay Island, from 60 to $150 \mathrm{~m}$, in the National Museum of the Philippines, Manila (not seen).

\section{Paratypes}

PHILIPPINES: 4 specimens in the former collection of E. de Suduiraut and Conchology, Inc., Mactan Island (not seen).

\section{Sequenced material}

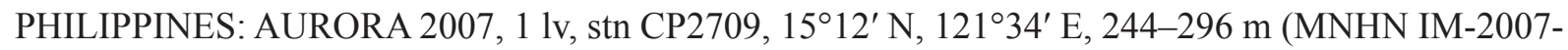
34608).

NEW CALEDONIA: CONCALIS, 1 lv, stn CP2960, North of New Caledonia, Grand Passage, $\left(19^{\circ} 05^{\prime}\right.$ S, $163^{\circ} 13^{\prime}$ E, 382-387 m (MNHN IM-2007-35734).

SOLOMON ISLANDS: SALOMON 2, 1 lv, stn DW2169, 0901' S, 159 $06^{\prime} \mathrm{E}, 100-200 \mathrm{~m}$ (MNHN IM-2007-32612).

SOCIETY ISLANDS: TARASOC, $2 \mathrm{lv}$, stn DW3441, 16 $6^{\circ} 43^{\prime} \mathrm{S}, 151^{\circ} 26^{\prime} \mathrm{W}, 350-360 \mathrm{~m}$ (MNHN IM-

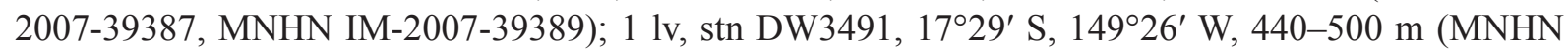
IM-2007-39262).

MADAGASCAR: ATIMO VATAE, 1 lv, stn DW3515, south Madagascar, $24^{\circ} 53^{\prime} \mathrm{S}, 4^{\circ} 28^{\prime} \mathrm{E}, 184$ 203 m (MNHN IM-2009-15090).

Other material examined (in MNHN if not otherwise mentioned)

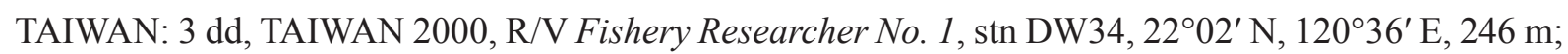
$1 \mathrm{dd}, \operatorname{stn} \mathrm{CP} 35,22^{\circ} 02^{\prime} \mathrm{N}, 120^{\circ} 27^{\prime} \mathrm{E}, 246 \mathrm{~m} ; 1 \mathrm{dd}, \operatorname{stn}^{\mathrm{D}} \mathrm{DW} 36,21^{\circ} 55^{\prime} \mathrm{N}, 120^{\circ} 36^{\prime} \mathrm{E}, 305 \mathrm{~m}$.

PHILIPPINES: $1 \mathrm{spm}$, Punta Engano, Mactan, in nets at 80-100 m (ANSP 456858); $1 \mathrm{spm}$, Olango, in lumun lumun nets, 40-100 m (ANSP 456859); $1 \mathrm{spm}$, Aliguay Island, trawled at $200 \mathrm{~m}$ (ANSP 456857); 4 spms, 'by boats from the Philippines' (ANSP 456854); 5 spms, same data as preceding (ANSP 456861); 2 spms, Balicasag Island, in tangle nets, 80-120 m (ANSP 456855); 2 spms, Aliguay Island, in coral rubble, $140 \mathrm{~m}$ (ANSP 456856); 2 spms, Aliguay Island, dredged on sandy/rocky mud, 160 m (ANSP 456860). - MUSORSTOM 3: 1 dd, stn CP96, 1400' N, 120¹8' E, 190-194 m.

ARAFURA SEA: KARUBAR: 5 dd, stn DW29, 05³6' S, 132 $56^{\prime}$ E, 181-184 m; 1 dd, stn DW32, $05^{\circ} 47^{\prime} \mathrm{S}, 132^{\circ} 51^{\prime} \mathrm{E}, 170-206 \mathrm{~m}$.

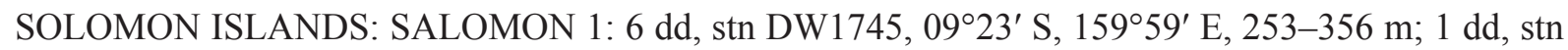
DW1758, 08 $49^{\prime} \mathrm{S}, 159^{\circ} 52^{\prime} \mathrm{E}, 180-187 \mathrm{~m} ; 1 \mathrm{lv}, 8 \mathrm{dd}$, stn DW1768, 08 $21^{\prime} \mathrm{S}, 160^{\circ} 42^{\prime} \mathrm{E}, 194-286 \mathrm{~m}$;

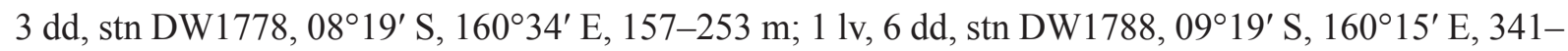

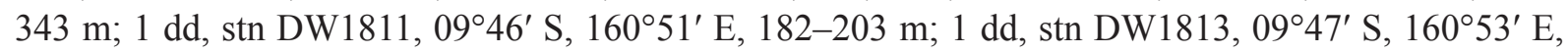
201-227 m; 3 dd, stn DW1820, 09 $52^{\prime}$ S, $160^{\circ} 51^{\prime}$ E, 256-329 m; 1 juv. dd, stn DW1834, $10^{\circ} 12^{\prime}$ S, $161^{\circ} 18^{\prime} \mathrm{E}, 225-281 \mathrm{~m}$.

PAPUA NEW GUINEA: KAVIENG 2014: 1 lv, stn DW4471, 0244' S, 150 38' E, 126-220 m (MNHN IM-2013-58726, not sequenced); 1 lv, stn DW4484, 02²6' S, 149 $55^{\circ}$ E, 229 m (MNHN IM-201358866, not sequenced). 
NEW CALEDONIA: BIOCAL: 1 dd, stn DW43, 22 $46^{\prime} \mathrm{S}, 167^{\circ} 15^{\prime} \mathrm{E}, 400 \mathrm{~m}$; 1 dd, stn DW82, 20³1' S, $166^{\circ} 50^{\prime}$ E, 440-460 m; 1 dd, stn DW83, 20³5' S, 166 $54^{\prime}$ E, 460 m. - BIOGEOCAL: 5 dd, stn KG252,

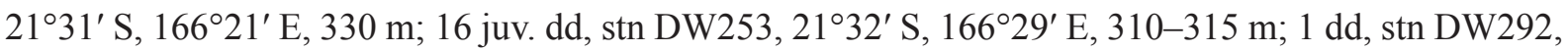
$20^{\circ} 28^{\prime} \mathrm{S}, 166^{\circ} 48^{\prime} \mathrm{E}, 465-470 \mathrm{~m}$; $3 \mathrm{dd}$, stn DW308, 2040' S, 166 58 $58^{\prime} \mathrm{E}, 510-590 \mathrm{~m}$. - SMIB 2: $2 \mathrm{dd}$, stn

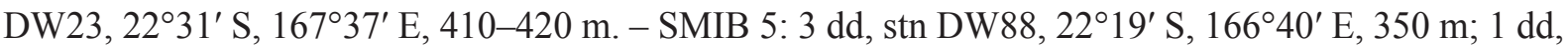

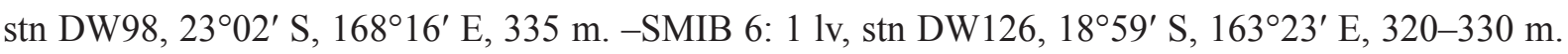

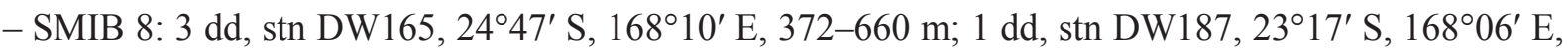

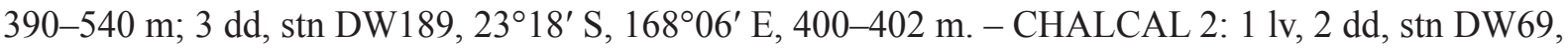
$24^{\circ} 44^{\prime} \mathrm{S}, 168^{\circ} 08^{\prime} \mathrm{E}, 260 \mathrm{~m}$. - BATHUS 1: 2 dd, stn DW683, 203' S, $165^{\circ} 07^{\prime} \mathrm{E}, 380-400 \mathrm{~m}$; $1 \mathrm{dd}$,

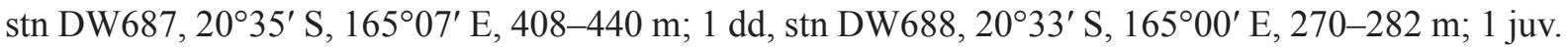

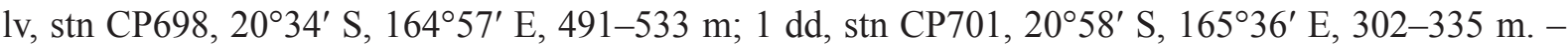

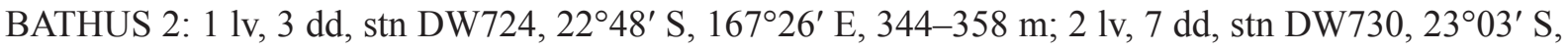

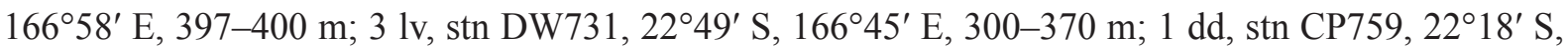
$166^{\circ} 10^{\prime}$ E, 370-420 m. - BATHUS 3: $8 \mathrm{lv}+\mathrm{dd}$, stn DW827, $23^{\circ} 22^{\prime} \mathrm{S}, 168^{\circ} 01^{\prime} \mathrm{E}, 381-469 \mathrm{~m}$; $1 \mathrm{lv}$, stn DW838, $23^{\circ} 01^{\prime} \mathrm{S}, 166^{\circ} 56^{\prime} \mathrm{E}, 400-402 \mathrm{~m}$. - BATHUS 4: 2 dd, stn DW885, 22 $05^{\prime} \mathrm{S}, 165^{\circ} 58^{\prime} \mathrm{E}$,

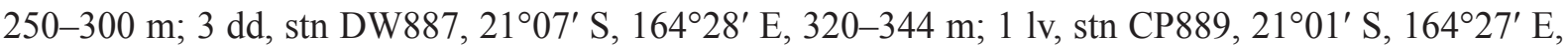

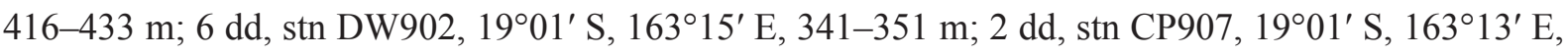
370-394 m; 1 dd, stn DW943, 20¹2' S, 164³1' E, 316-347 m. - BERYX 11: 15 lv+dd, stn DW11/CP23, $24^{\circ} 43^{\prime}-24^{\circ} 44^{\prime} \mathrm{S}, 168^{\circ} 08^{\prime}-168^{\circ} 10^{\prime} \mathrm{E}, 270-350 \mathrm{~m}$. - MUSORSTOM 5: $2 \mathrm{lv}, 3 \mathrm{dd}, \mathrm{stn} 301,2^{\circ} 07^{\prime} \mathrm{S}$, $159^{\circ} 25^{\prime}$ E, 487-610 m, Nova Bank. - MUSORSTOM 6: 3 lv, 3 dd, stn DW391, 2047' S, $167^{\circ} 06^{\prime} \mathrm{E}$,

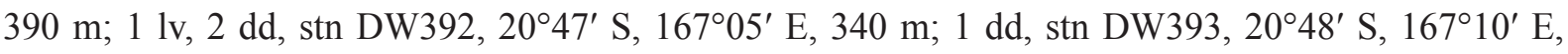

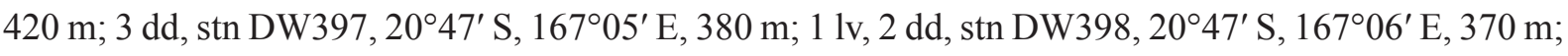

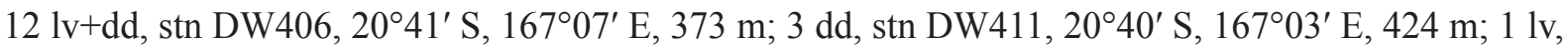

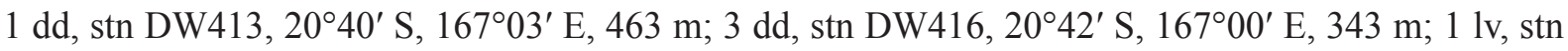

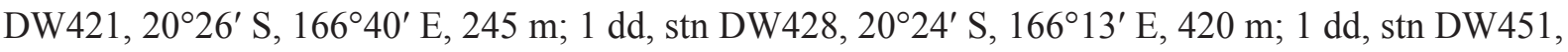

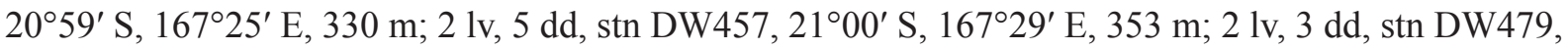

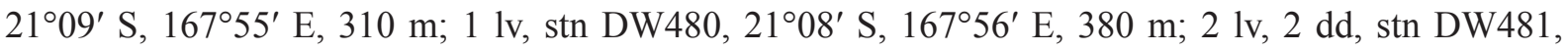

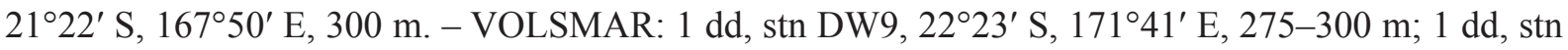
DW38, $22^{\circ} 22^{\prime} \mathrm{S}, 168^{\circ} 44^{\prime} \mathrm{E}, 380-420 \mathrm{~m}$; 1 dd, stn DW51, 205' $\mathrm{S}, 170^{\circ} 03^{\prime} \mathrm{E}, 450 \mathrm{~m}$. - NORFOLK 1 : 3 lv, stn DW1680, 244ㄴ S, $168^{\circ} 10^{\prime}$ E, 385-392 m.

VANUATU: MUSORSTOM 8: 1 lv, stn DW963, 20²0' S, 169 49' E, 400-440 m; 1 dd, stn DW964,

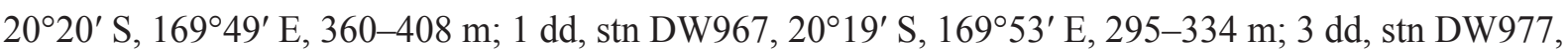
$19^{\circ} 25^{\prime} \mathrm{S}, 169^{\circ} 29^{\prime} \mathrm{E}, 410-505 \mathrm{~m} ; 8$ dd, stn DW978, $19^{\circ} 23^{\prime} \mathrm{S}, 169^{\circ} 27^{\prime} \mathrm{E}, 408-413 \mathrm{~m} ; 3$ dd, stn DW988, $19^{\circ} 16^{\prime} \mathrm{S}, 169^{\circ} 24^{\prime} \mathrm{E}, 372-466 \mathrm{~m} ; 1 \mathrm{dd}$, stn DW1060, $16^{\circ} 14^{\prime} \mathrm{S}, 167^{\circ} 21^{\prime} \mathrm{E}, 375-397 \mathrm{~m} ; 3 \mathrm{dd}$, stn DW1065, $16^{\circ} 16^{\prime} \mathrm{S}, 167^{\circ} 21^{\prime} \mathrm{E}, 360-419 \mathrm{~m}$.

WALLIS AND FUTUNA: MUSORSTOM 7: 3 lv(?), stn DW510, $14^{\circ} 14^{\prime} \mathrm{S}, 178^{\circ} 11^{\prime} \mathrm{W}, 280-370 \mathrm{~m}$.

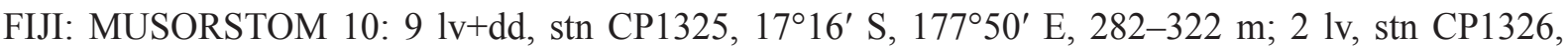

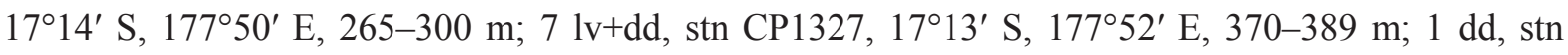

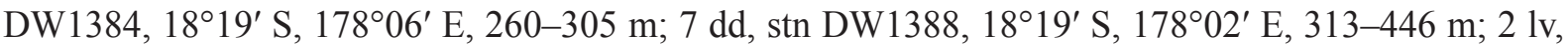
stn CP1389, $18^{\circ} 19^{\prime} \mathrm{S}, 178^{\circ} 05^{\prime} \mathrm{E}, 241-417 \mathrm{~m}$; 1 lv, 2 dd, stn CP1390, $18^{\circ} 19^{\prime} \mathrm{S}, 1^{\circ} 8^{\circ} 05^{\prime} \mathrm{E}, 234-361 \mathrm{~m}$.

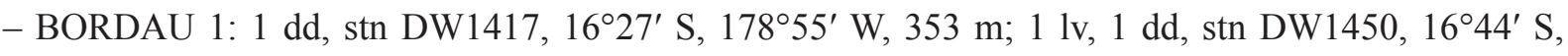

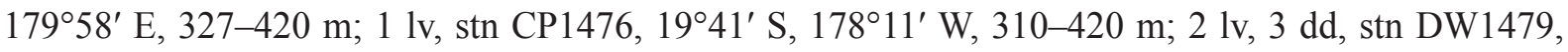
$20^{\circ} 58^{\prime} \mathrm{S}, 178^{\circ} 45^{\prime} \mathrm{W}, 450-460 \mathrm{~m}$; 2 lv, stn CP1481, 20 $57^{\prime} \mathrm{S}, 178^{\circ} 45^{\prime} \mathrm{W}, 441-506 \mathrm{~m} ; 2$ lv, stn DW1496, $18^{\circ} 43^{\prime} \mathrm{S}, 178^{\circ} 23^{\prime} \mathrm{W}, 392-407 \mathrm{~m} ; 3$ dd, stn DW1499, $18^{\circ} 40^{\prime} \mathrm{S}, 178^{\circ} 27^{\prime} \mathrm{W}, 389-400 \mathrm{~m}$; 1 lv, stn CP1500, $18^{\circ} 42^{\prime} \mathrm{S}, 178^{\circ} 26^{\prime} \mathrm{W}, 366-389 \mathrm{~m}$. 
TONGA: BORDAU 2: 2 dd, stn DW1518, 21 ${ }^{\circ} 21^{\prime} \mathrm{S}, 175^{\circ} 07^{\prime} \mathrm{W}, 336-347 \mathrm{~m} ; 2 \mathrm{dd}, \operatorname{stn} \mathrm{DW} 1534,2^{\circ} 43^{\prime} \mathrm{S}$,

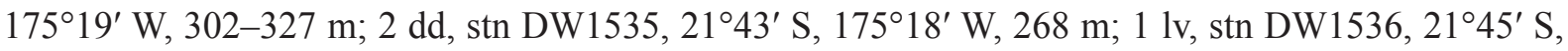

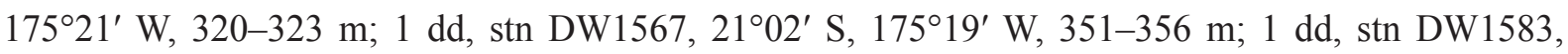

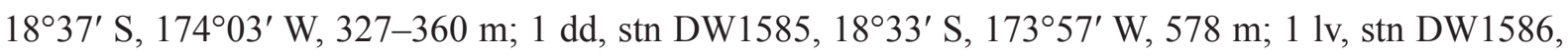

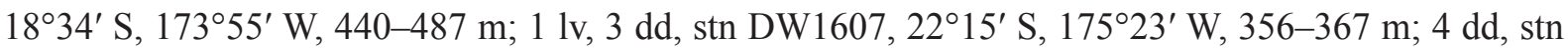

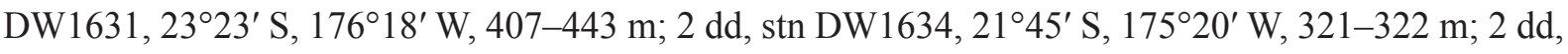

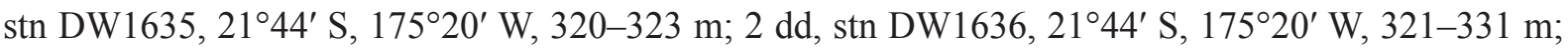
1 lv, stn CP1641, $21^{\circ} 09^{\prime} \mathrm{S}, 175^{\circ} 22^{\prime} \mathrm{W}, 395 \mathrm{~m}$,

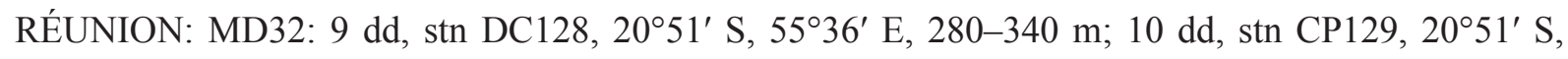
$55^{\circ} 36^{\prime}$ E, 290-300 m; 1 dd, stn DC136, 2046' S, 55³6' E, 915-922 m.

\section{Description}

Shell slender, fusiform, small to medium-sized for genus (attaining $30.8 \mathrm{~mm}$, ANSP 456861). Protoconch multispiral of 4.25 whorls (Fig. 3K), conical, smooth, except scattered microgranules more dense along suture, flesh coloured with reddish thin line along suture. Whorls somewhat convex, last $1 / 2$ whorl with 11-12 rounded axial riblets progressing in strength, protoconch/teleoconch boundary without varix, defined by appearance of spiral sculpture. Protoconch diameter $1000 \mu \mathrm{m}$, exposed height $1240 \mu \mathrm{m}$. Teleoconch whorls convex, suture deep, adpressed. Axial sculpture of very prominent ribs, 7-9 per whorl. Spiral sculpture of evenly spaced strong cords, 2 on first whorl, 3-4 on penultimate whorl, 8-9 on last whorl and shell base, becoming much weaker on canal. Weaker but distinct secondary cords between primary cords, one on earliest whorls increasing to 5 on last whorl, primary and secondary cords becoming similar in size on canal. Siphonal canal long, open, straight, about $1 / 3$ of shell length. Aperture with 7-8 smooth teeth periodically appearing and corresponding to interspaces between axial ribs. Inner lip adherent, forming smooth, white columellar callus, 2-3 distinct columellar rounded plicae, adapical two more prominent than abapical one. Teleoconch light brown, usually with lighter spiral band on whorls periphery.

Radula (MNHN IM-2013-58726, SL 14.1, AL 3.1 mm, Fig. 4 B) 2.13 mm in length (69\% of AL), of about 125 transverse rows of teeth, of which 30 nascent. Radula narrow, distance between outer edges of lateral teeth about $50 \mu \mathrm{m}$ (1.6\% of AL without canal). Lateral teeth medium broad, arcuate, with attenuated outer anterior corner, producing long 'handle'. Lateral teeth with three cusps on right side and four on left side, subequal in length, closely spaced, slightly curved and an additional small but distinct innermost cusp. Inner cusp(s) slightly narrower than lateral ones. Central tooth small, long trapezoid with narrower base, with three cusps, central one nearly twice as long as outer ones.

\section{Distribution}

Very widely distributed: in Indo-Pacific, from Taiwan and the Philippines, to French Polynesia, Madagascar and off Réunion. Recorded at depths from 170 to 920 m, alive in 225-610 m. Unconfirmed depths from commercial sources in the Philippines from $40 \mathrm{~m}$.

\section{Remarks}

The species has a smooth columella and therefore is well differentiated from its congeners with columellar plicae. Besides, G. williami comb. nov. has a small, rather slender shell with attenuated straight canal. It is most similar to Granulifusus norfolkensis sp. nov., differing in details of colouration and in having a straight siphonal canal rather than inclined to left. Molecular data clearly separate the two species. 
Granulifusus jeanpierrevezzaroi (Cossignani, 2017) comb. nov.

Figs $4 \mathrm{C}-\mathrm{D}, 5$

Pseudolathyrus cf. kurodai Springsteen \& Leobrera, 1986: 177, pl. 48 fig. 1.

Fusolatirus kuroseanus Thach, 2007: 118, pl. 29, no. 518 (not of Okutani 1975).

Pseudolatirus jeanpierrevezzaroi Cossignani, 2017: 14-15.

Pseudolatirus jeanpierrevezzaroi - Mallard \& Robin 2017: 152.

\section{Molecular diagnosis}

A molecular diagnosis is given in Table 2.

\section{Type material}

Holotype

VIETNAM: off Nha Trang, SL $66.3 \mathrm{~mm}$ (MMM) (not seen).

\section{Sequenced material}

NEW CALEDONIA: TERRASSES: $1 \mathrm{lv}$, stn CP3095, 22 $02^{\prime} \mathrm{S}, 167^{\circ} 06^{\prime} \mathrm{E}, 320-380 \mathrm{~m}$ (MNHN IM2007-36886). - CONCALIS: 1 lv, stn CP3026, Grand Passage, 20¹7' S, 16350' E, 590-809 m (MNHN IM-2007-35083).

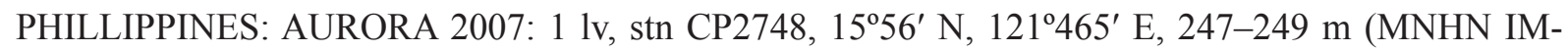
2007-34593).

\section{Other material examined}

SOUTH AFRICA: 2 spms, Agulhas Bank, 160 m (ANSP 425332); 1 spm, Agulhas Bank, 160 m (ANSP 425328); 3 spms, off Durban (ANSP 425336).

TAIWAN: 2 spms, trawled (ANSP 425339); 2 spms, 'by divers under rocks, southeast Sea of Formosa' (ANSP 425338); 2 spms, SW of Taiwan, 140-200 m (ANSP 425335); 2 spms, 200-300 m (ANSP 425333); 1 spm, China Sea, off Taiwan (ANSP 425329); 4 spms, trawled (ANSP 425334).

MOZAMBIQUE: 3 spms (ANSP 425340).

VIETNAM: 1 spm, Nha Trang region, 20-35 m (ANSP 425341).

SOUTH CHINA SEA: 2 spms, on sand bottom (ANSP 422786); 1 spm, 'deep water' (ANSP 422785).

PHILIPPINES: 3 spms, off Bohol, tangle nets (ANSP 425330); 1 spm, Philippine Is (ANSP 425331); $1 \mathrm{dd}$, off Mindanao (ANSP 425337). - MUSORSTOM 2: 5 lv, stn CP02, $14^{\circ} 01^{\prime} \mathrm{N}, 120^{\circ} 17^{\prime} \mathrm{E}, 184-$ $186 \mathrm{~m} ; 37 \mathrm{lv}+\mathrm{dd}, \operatorname{stn} \mathrm{CP} 26,13^{\circ} 49^{\prime} \mathrm{N}, 120^{\circ} 50^{\prime} \mathrm{E}, 299-320 \mathrm{~m} ; 18 \mathrm{lv}+\mathrm{dd}, \operatorname{stn} \mathrm{CP} 68,14^{\circ} 01^{\prime} \mathrm{N}, 120^{\circ} 18^{\prime} \mathrm{E}$,

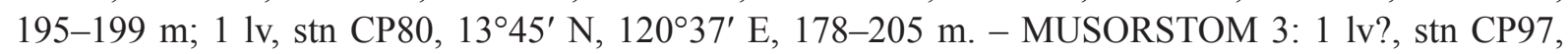
$14^{\circ} 00^{\prime} \mathrm{N}, 120^{\circ} 18^{\prime} \mathrm{E}, 189-194 \mathrm{~m}$; $5 \mathrm{lv}, \mathrm{stn} \mathrm{CP} 112,1^{\circ} 00^{\prime} \mathrm{N}, 120^{\circ} 18^{\prime} \mathrm{E}, 187-199 \mathrm{~m}$.

NEW CALEDONIA: 'Vauban' 1978-79: 1 dd, stn DR34, 22 $32^{\prime}$ S, $166^{\circ} 26^{\prime}$ E, 350-420 m; 1 lv, stn DR40, $22^{\circ} 30^{\prime} \mathrm{S}, 166^{\circ} 24^{\prime} \mathrm{E}, 250-350 \mathrm{~m}$. - LAGON: 1 lv, stn $1062,20^{\circ} 15^{\prime} \mathrm{S}, 163^{\circ} 53^{\prime} \mathrm{E}, 300-320 \mathrm{~m}$. -

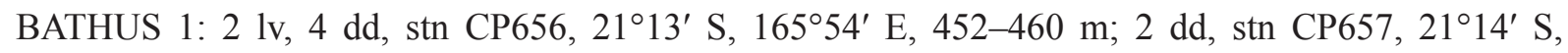
$165^{\circ} 55^{\prime} \mathrm{E}, 490-530 \mathrm{~m} ; 1 \mathrm{dd}, \mathrm{stn} \mathrm{CP} 669,20^{\circ} 57^{\prime} \mathrm{S}, 1^{\circ} 5^{\circ} 35^{\prime} \mathrm{E}, 255-280 \mathrm{~m} ; 1 \mathrm{lv}, 1 \mathrm{dd}, \mathrm{stn}$ CP670, $20^{\circ} 54^{\prime} \mathrm{S}, 165^{\circ} 53^{\prime} \mathrm{E}, 394-397 \mathrm{~m}$; $1 \mathrm{dd}$, stn DW683, 20³5' S, $165^{\circ} 07^{\prime} \mathrm{E}, 380-400 \mathrm{~m} ; 8 \mathrm{lv}, 1 \mathrm{dd}$, stn

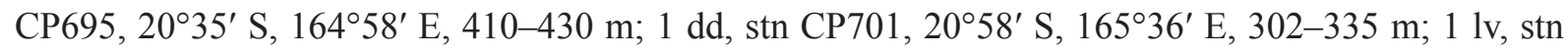




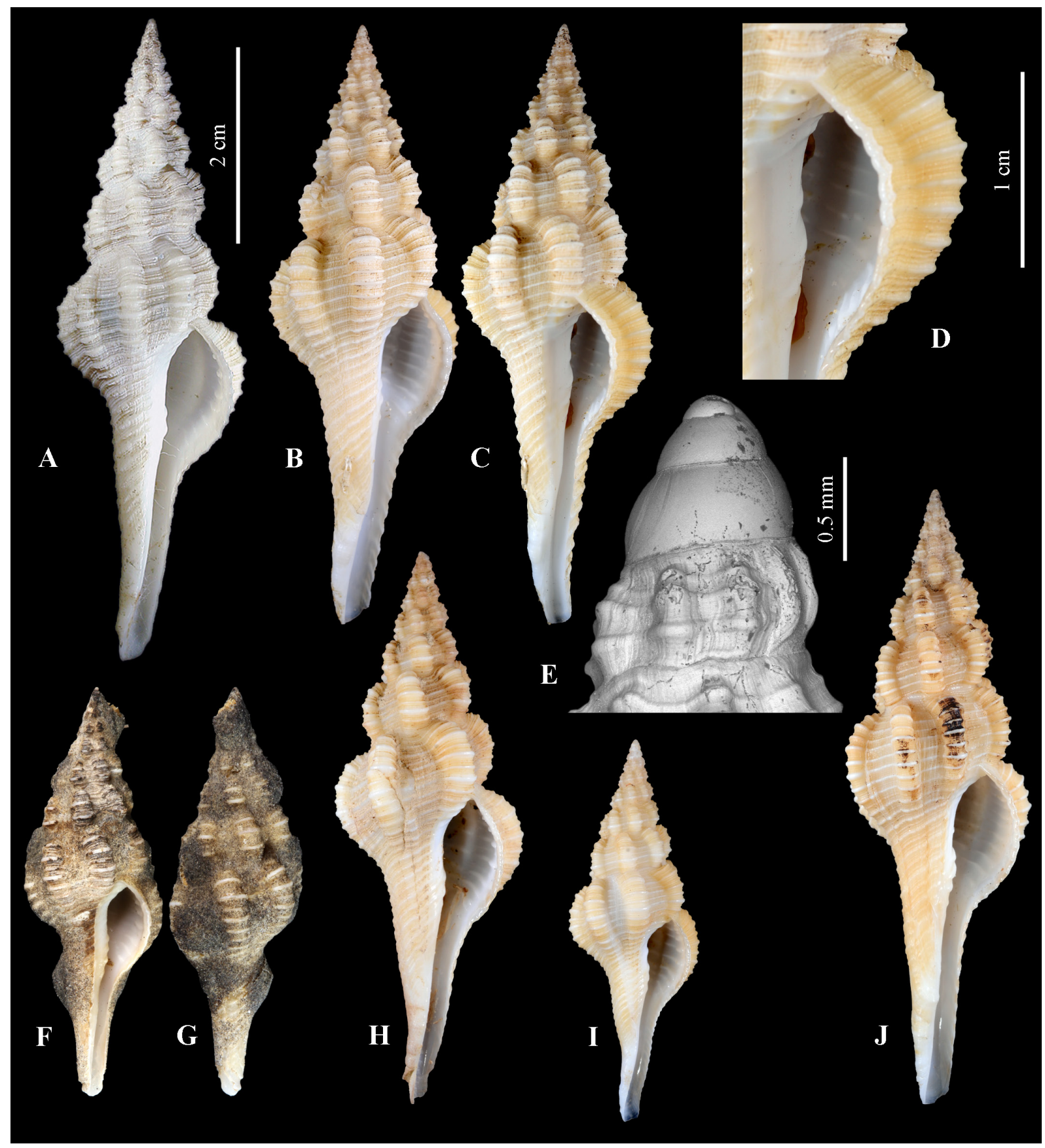

Fig. 5. Granulifusus jeanpierrevezzaroi (Cossignani, 2017) comb. nov. A. Holotype, SL $66.3 \mathrm{~mm}$ (MMM) (photo courtesy of T. Cossignani). B-D. Sequenced specimen, SL $62.2 \mathrm{~mm}$ (MNHN IM-200734593). D. Enlarged view of columella to show the plicae. E. Protoconch, New Caledonia, BATHUS 1, stn CP713, 21 ${ }^{\circ} 45^{\prime} \mathrm{S}, 166^{\circ} 37^{\prime} \mathrm{E}, 250 \mathrm{~m}$. F-G. Philippines, MUSORSTOM 3, stn CP97, $14^{\circ} 00^{\prime} \mathrm{N}$, $120^{\circ} 18^{\prime} \mathrm{E}, 189-194 \mathrm{~m}$, SL $42.2 \mathrm{~mm}$. H. Fiji, MUSORSTOM 10, stn CP1355, 1750' S, $178^{\circ} 49^{\prime} \mathrm{E}$, 302-310 m, SL 56.8 mm. I. New Caledonia, BATHUS 1, stn CP670, 2054' S, 16553' E, 394-397 m, SL 39.4 mm. J. Philippines, MUSORSTOM 2, stn CP68, 1401' N, 120¹8' E, 195-199 m, SL 63.7 mm. All shells to the same scale, except D-E. 


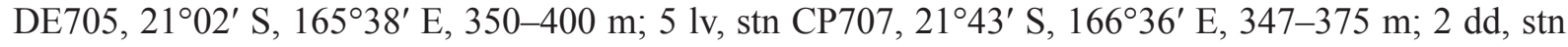

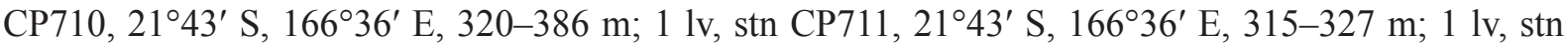
CP712, $21^{\circ} 44^{\prime} \mathrm{S}, 166^{\circ} 35^{\prime} \mathrm{E}, 210 \mathrm{~m}$; 1 lv, stn CP713, $21^{\circ} 45^{\prime} \mathrm{S}, 166^{\circ} 37^{\prime} \mathrm{E}, 250 \mathrm{~m}$. - BATHUS 4: 3 lv, stn CP946, 20³4' S, 16458' E, 386-430 m; 3 lv, stn CP947, 20³4' S, 16458' E, 470-490 m.

FIJI: MUSORSTOM 10: 6 dd, stn CP1320, $17^{\circ} 17^{\prime} \mathrm{S}, 1^{\circ} 7^{\circ} 54^{\prime} \mathrm{E}, 290-300 \mathrm{~m} ; 1 \mathrm{lv}, 2 \mathrm{dd}, \operatorname{stn} \mathrm{CP} 1325$,

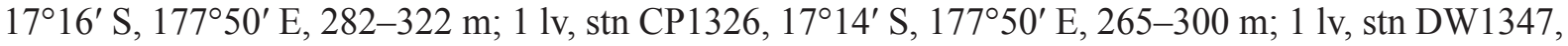

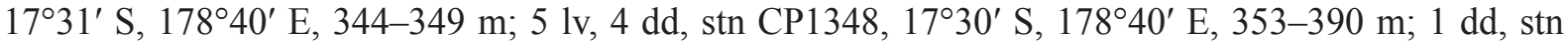

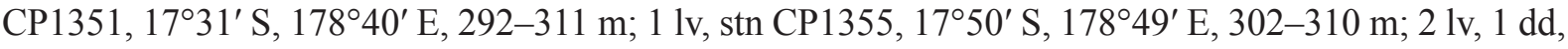
stn CP1360, $18^{\circ} 00^{\prime} \mathrm{S}, 178^{\circ} 48^{\prime} \mathrm{E}, 402-444 \mathrm{~m} ; 3$ lv, stn CP1368, $18^{\circ} 11^{\prime} \mathrm{S}, 178^{\circ} 24^{\prime} \mathrm{E}, 380-469 \mathrm{~m} ; 1 \mathrm{dd}$, stn CP1369, $18^{\circ} 11^{\prime} \mathrm{S}, 178^{\circ} 23^{\prime} \mathrm{E}, 392-433 \mathrm{~m}$.

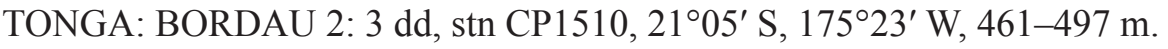

\section{Description}

Shell slender, fusiform, large for genus (to $90.0 \mathrm{~mm}$, ANSP 425335). Protoconch multispiral, conical, smooth, translucent pale grey, of 3.25 somewhat convex whorls, last $1 \frac{1}{2}$ whorl with $4-6$ rounded axial riblets progressing in strength, protoconch/teleoconch boundary without varix. Protoconch diameter $1100 \mu \mathrm{m}$, exposed height $1280 \mu \mathrm{m}$ (Fig. 5E). Teleoconch whorls convex, suture deep, adpressed. Axial sculpture of very prominent ribs, 7-9 per whorl. Spiral sculpture of evenly spaced strong cords, 2-3 on first whorl, $6-8$ on penultimate whorl, about $23-25$ on last whorls, becoming evanescent towards tip of canal. Weaker secondary cords between primary strong cord pairs, 1 on earliest whorls increasing to 3 on later whorls. Siphonal canal long, open, straight, about $1 / 3$ of shell length. Aperture with $7-12$ interior lirae. Inner lip adherent, forming smooth, white columellar callus, with 3 rounded columellar plicae, adapical two more prominent than abapical one. In side view additional adapicalmost weak plicae can be observed. Teleoconch pale flesh to pale orange-brown with lighter prominent axial cords. Periostracum medium to dark brown.

Radula (MNHN IM-2007-34593, $62.2 \mathrm{~mm}$, Fig. 4C-D) $2.6 \mathrm{~mm}$ in length (13\% of AL), of more than 105 transverse rows of teeth, of which 37 nascent. Radula narrow, distance between outer edges of lateral teeth about $130 \mu \mathrm{m}(0.67 \%$ of AL without canal). Lateral teeth medium broad, arcuate, with attenuated outer anterior corner, producing short 'handle', and with six cusps, subequal in length, closely spaced, slightly curved and one additional small but distinct innermost cusp. Central tooth small, trapezoid with narrower base, with three cusps, central narrowest. Narrow but distinct transverse folds of the membrane situated serially between rows of lateral teeth (arrow). The radula of an additional specimen was examined and it is generally similar, although the left lateral teeth have five cusps, while vs six on the right teeth; also the central teeth are relatively narrower and longer, tricuspid with central cusp longest.

Body morphology and digestive system anatomy are in all details similar to Angulofusus nedae Fedosov \& Kantor, 2012, the major difference being the longer and thinner proboscis, which attains $20 \mathrm{~mm}$ in studied specimen (2.2 AL without canal) and is coiled within rhynchodeum.

\section{Distribution}

Throughout the tropical Indo-West Pacific, at depths 140-809 m, one specimen from commercial sources said to have been collected in 20-35 m (Vietnam).

\section{Remarks}

Conchologically the species is most similar to Granulifusus annae sp. nov. which has a similar size, but G. jeanpierrevezzaroi comb. nov. differs in having fewer axial ribs per whorl and being nearly uniformly 
coloured with lighter spiral cords, while in Granulifusus annae sp. nov. the spiral cords are darker than the background. These two species also differ in radular morphology.

Granulifusus jeanpierrevezzaroi comb. nov. is a very common species in the Philippines and is found on soft bottoms throughout the tropical Indo-West Pacific. It is remarkable that the species still had to be named, although it had been illustrated several times under incorrect names. Shells of many specimens are covered with zoantharians (e.g., Fig. 5F-G).

Granulifusus annae sp. nov.

urn:1sid:zoobank.org:act:76CA2DC8-A32A-4164-9218-8B50E9AEB780

Figs 4E, 6

Pseudolatirus kurodai Couto et al., 2016: table 1, fig. 6J (not of Okutani \& Sakurai 1964).

Pseudolatirus kuroseanus Couto, 2016: 41, fig. 28 (not of Okutani 1975). [The shell depicted under the same name on fig. 27 was accidentally switched in the collection with the shell of sequenced specimen MNHN IM-2013-14709]

\section{Molecular diagnosis}

A molecular diagnosis is given in Table 2.

\section{Diagnosis}

Shell thin but solid, slender, fusiform, up to $55 \mathrm{~mm}$, protoconch multispiral of 2.25 whorls. Teleoconch whorls convex, axial sculpture of broad, raised axial ribs, spiral sculpture of evenly spaced, strong cords, 35 on last whorl. Aperture lirate inside, columella with several weak plicae. Teleoconch light tan with darker spiral cords.

\section{Etymology}

The specific epithet pays homage to Ann Snyder, in fitting tribute for this elegant and beautiful species.

\section{Type material}

\section{Holotype}

PAPUA NEW GUINEA: Rempi Area, $05^{\circ} 03^{\prime} \mathrm{S}, 145^{\circ} 49^{\prime} \mathrm{E}, 120 \mathrm{~m}$, expedition PAPUA NIUGINI, stn PP08, sequenced (Fig. 6A-C) (MNHN IM-2013-14709).

\section{Sequenced material}

NEW CALEDONIA: EXBODI: 1 lv, stn CP3823, 21ํ5' S, 166 55' E, 246-255 m (MNHN IM-201342520).

PHILIPPINES: AURORA 2007: 3 lv, stn CP2716, 14³ $30^{\prime} \mathrm{N}, 121^{\circ} 41^{\prime} \mathrm{E}, 335-356 \mathrm{~m}$ (MNHN IM-2007-

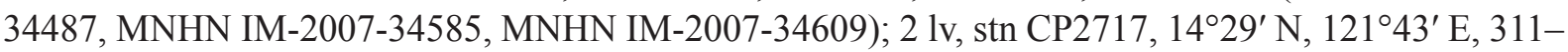
361 m (MNHN IM-2007-34486, MNHN IM-2007-34586).

Other material examined (all in MNHN)

VANUATU: MUSORSTOM 8: 2 dd, stn DW1003, 1849' S, 168 59' E, 200-327 m; 1 lv, stn CP1123, $15^{\circ} 07^{\prime} \mathrm{S}, 166^{\circ} 55^{\prime} \mathrm{E}, 262-352 \mathrm{~m}$.

NEW CALEDONIA: HALIPRO 1: 1 dd, stn CP853, $21^{\circ} 45^{\prime} \mathrm{S}, 166^{\circ} 37^{\prime} \mathrm{E}, 241-250 \mathrm{~m}$; 2 juv. dd, stn CC855, $21^{\circ} 45^{\prime} \mathrm{S}, 166^{\circ} 37^{\prime} \mathrm{E}, 204-220 \mathrm{~m} ; 2$ dd, 1 juv. dd, stn CP866, $21^{\circ} 26^{\prime} \mathrm{S}, 166^{\circ} 17^{\prime} \mathrm{E}, 550-600 \mathrm{~m}$. BATHUS 1: $1 \mathrm{lv}$ (radula examined), stn CP669, 20 $0^{\circ} 7^{\prime} \mathrm{S}, 1^{\circ} 5^{\circ} 35^{\prime} \mathrm{E}, 255-280 \mathrm{~m} ; 2 \mathrm{dd}$, stn CP695, 


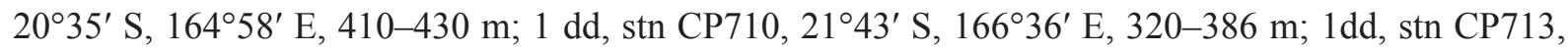
$21^{\circ} 45^{\prime} \mathrm{S}, 166^{\circ} 37^{\prime} \mathrm{E}, 250 \mathrm{~m}$.

LOYALTY RIDGE: MUSORSTOM 6: 1 lv(?), stn DW449, 2054' S, 167²18' E, 300 m.

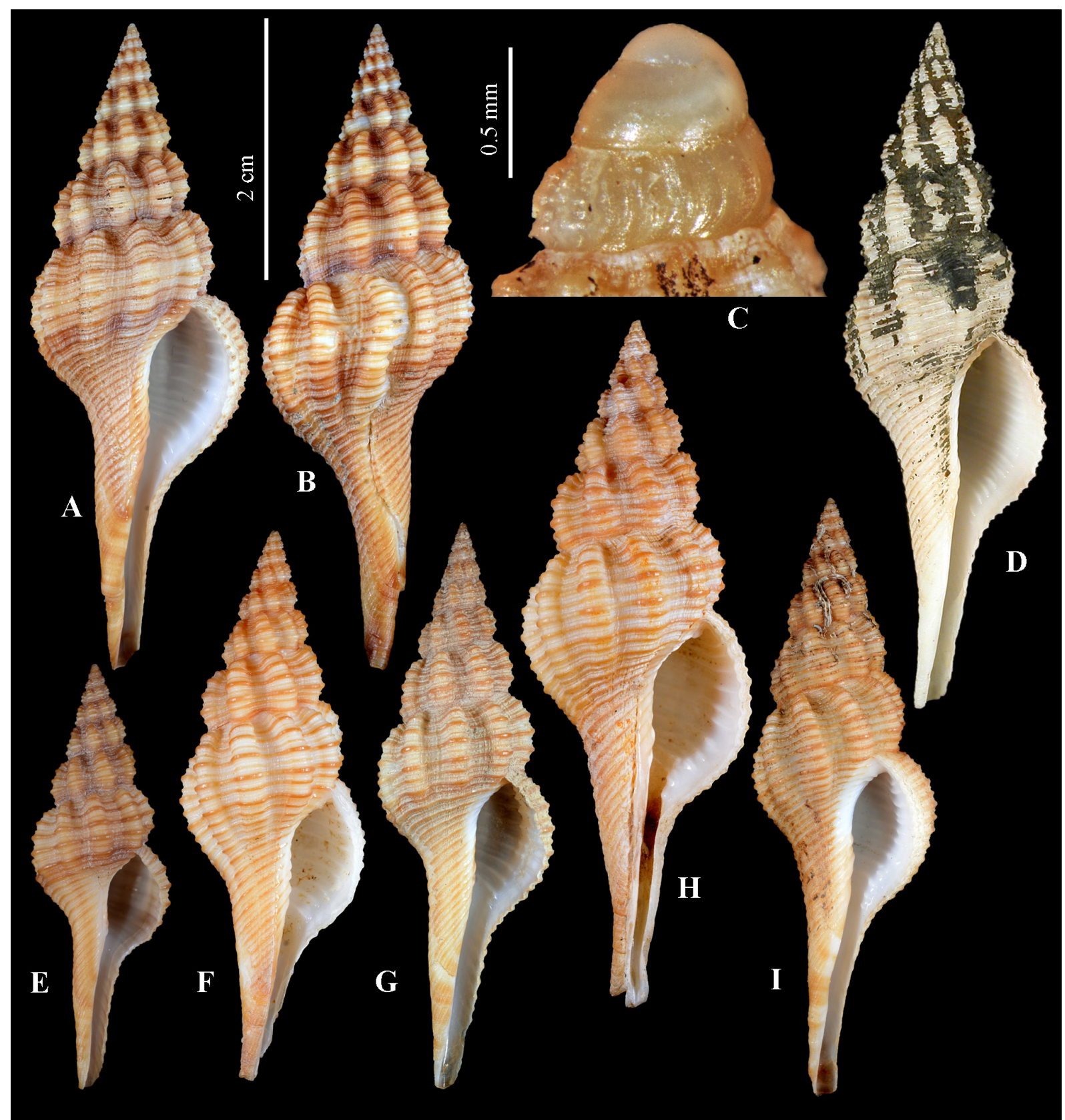

Fig. 6. Granulifusus annae sp. nov. A-C. Holotype, sequenced, SL 50.3 mm (MNHN IM-201314709). C. Protoconch. D. Philippines, SL 54 mm, sequenced specimen (MNHN IM-2007-34486). E. New Caledonia, SL $23.1 \mathrm{~mm}$, sequenced specimen (MNHN IM-2013-42520). F-G. New Caledonia, BATHUS 1, stn CP669, 20 $0^{\circ} 57^{\prime} \mathrm{S}, 165^{\circ} 35^{\prime} \mathrm{E}, 255-280 \mathrm{~m}$. F. SL $42.7 \mathrm{~mm}$ (radula, see Fig. 4E). G. SL

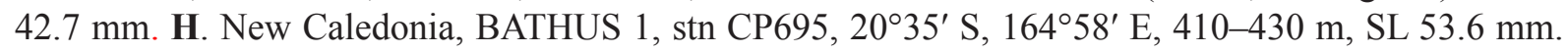
I. Vanuatu, MUSORSTOM 8, stn CP1123, $15^{\circ} 07^{\prime} \mathrm{S}, 166^{\circ} 55^{\prime} \mathrm{E}, 262-352 \mathrm{~m}$, SL $46.8 \mathrm{~mm}$. All shells to the same scale. 


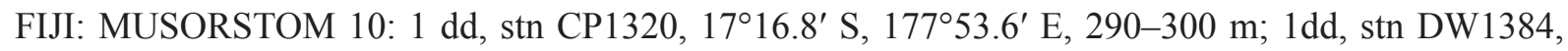
$18^{\circ} 18.5^{\prime} \mathrm{S}, 178^{\circ} 05.8^{\prime} \mathrm{E}, 260-305 \mathrm{~m}$. - BORDAU 1: $1 \mathrm{lv}(?), 2 \mathrm{dd}$, stn CP1402, $16^{\circ} 38^{\prime} \mathrm{S}, 179^{\circ} 36^{\prime} \mathrm{E}$, 260-279 m; 1lv, 1dd, stn CP1403, 16²40' S, 179³6' E, 220-224 m.

\section{Description}

Shell thin, slender, fusiform, consisting of 2.25 protoconch and 8.5 teleoconch whorls (in holotype). Protoconch bluntly conical (Fig. 6C), with moderately convex whorls, last $1 / 2$ whorl with 6 axial riblets, protoconch/teleoconch discontinuity distinct. Protoconch diameter $930 \mu \mathrm{m}$, exposed height $850 \mu \mathrm{m}$. Teleoconch whorls convex, suture deep, impressed. Axial sculpture of broad, raised axial ribs, running from suture to suture, in holotype 9 on first whorl, 8 on $2^{\text {nd }-6^{\text {th }}}$ whorls and 9 on last whorl. Spiral sculpture of evenly spaced, strong, raised cords, 3-4 on earlier whorls, abapicalmost strongest, 6 on antepenultimate and penultimate whorls, 35 on last whorl. Between major cords, 1-3 smaller secondary cords, slightly nodulose at intersection with raised growth lines. Siphonal canal open, long, straight. Aperture with 12 weak lirae inside. Inner lip completely adherent to columella, forming very thin, shiny columellar callus with 5 weak columellar plicae. No umbilicus. Protoconch light brown, interior of aperture white. Teleoconch light-tan with still lighter spiral band on shell periphery, spiral cords tan, usually darker than background, upper spiral whorls brownish adapically.

Operculum small, length in holotype $5.3 \mathrm{~mm}$, occupying slightly more than $1 / 3$ of aperture without canal, oval, nucleus supposingly terminal, older part of operculum missing.

Holotype measurements: SL $50.3 \mathrm{~mm}$, AL (with canal) $30.0 \mathrm{~mm}$, SW $15.9 \mathrm{~mm}$.

Radula (Fig. 4E, New Caledonia, BATHUS 1, stn CP669, 20 $57^{\prime} \mathrm{S}, 165^{\circ} 35^{\prime} \mathrm{E}, 255-280 \mathrm{~m}$, shell on Fig. $6 \mathrm{~F})$ narrow, distance between outer edges of lateral teeth about $60 \mu \mathrm{m}(0.48 \%$ of AL without canal $)$. Lateral teeth medium broad, arcuate, with attenuated outer anterior corner, producing medium long 'handle'. Lateral teeth with outermost very short small cusp (Fig. 4E, black arrows), four broadly spaced and slightly recurved, long cusps, subequal in length. Inner anterior corner also attenuated, forming a triangular outgrowth, sometimes bearing indistinct an additional innermost small cusp. Central tooth small, very narrow (length to width ratio 5), with three short and blunt cusps, central longest. Narrow but distinct transverse folds of membrane situated serially between rows of lateral teeth, on both sides of central teeth (white arrow).

The radula of the holotype was examined and illustrated by Couto (2016: fig. 28) under the name Pseudolatirus kuroseanus. It is in all details similar to the radula figured here, the minor differences are that the outermost small cusp is more distinct, the innermost outgrowth bears a distinct small cusp, and the cusps of the central teeth are sharp rather than blunt.

\section{Distribution}

Philippines, New Caledonia, Papua New Guinea, Vanuatu, Fiji, 120-600 m, alive in 120-360 m.

\section{Remarks}

This species is moderately variable both in slenderness of the shell and in whorl convexity. Rarely the spiral cords are lighter than the shell background (observed in sequenced juvenile specimen MNHN IM-2007-34609).

This new species is similar to G. kurodai comb. nov. and has been confused with it, but can be distinguished by a different protoconch, consisting of 2.25 whorls in $G$. annae sp. nov. rather than only 1.75 whorls in G. kurodai comb. nov., as well as by a straight siphonal canal, darker shell coloration, and more pronounced and less numerous primary spiral cords (6 on penultimate whorl in G. annae 
sp. nov. and 10 in paratype of G. kurodai comb. nov.). The species is also conchologically similar to G. jeanpierrevezzaroi comb. nov., differing in the more brownish background shell colour with more contrastingly coloured spiral cords, more dense axial ribs and a smaller protoconch. The molecular analysis clearly differentiates these species.

The radula of the new species is somewhat different from its congeners in having longer and more broadly spaced cusps on the lateral teeth.

Granulifusus norfolkensis sp. nov.

urn:1sid:zoobank.org:act:7483C94B-51FD-44D8-B117-B7140B65F385

Fig. 7A-D

\section{Diagnosis}

Shell thin, slender, fusiform, up to $14 \mathrm{~mm}$, protoconch of 4 whorls. Teleoconch whorls weakly convex, axial sculpture of narrow, broadly spaced, axial ribs, spiral sculpture of evenly spaced, strong primary cords, 7 on last whorl and weaker secondary cords between them. Aperture weakly lirate inside, columella with several distinct columellar plicae. Teleoconch light tan with several brown spiral bands present, also on siphonal canal.

\section{Etymology}

The specific epithet refers to the collecting locality, the Norfolk Ridge, where the first known specimen of this species was collected.

\section{Type material}

Holotype

NEW CALEDONIA: north of Ile des Pins, $22^{\circ} 28^{\prime}$ S, $167^{\circ} 29^{\prime}$ E, 404-405 m, expedition KANACONO, stn DW4685, sequenced (MNHN IM-2013-68811).

\section{Other material examined}

NEW CALEDONIA: 1 lv, TERRASSES, stn DW3063, Norfolk Ridge, $23^{\circ} 23^{\prime} \mathrm{S}, 168^{\circ} 00^{\prime} \mathrm{E}, 430-480 \mathrm{~m}$, sequenced (MNHN IM-2007-36921).

\section{Description}

Shell thin, slender, fusiform, of nearly 4 protoconch and 5 teleoconch whorls (in holotype). Protoconch acutely conical (Fig. 7C), with moderately convex whorls, last $1 / 2$ whorl with 6 axial, progressively thickening, riblets, protoconch/teleoconch transition abrupt, marked by onset of distinctive sculpture. Protoconch diameter $1130 \mu \mathrm{m}$, exposed height $1150 \mu \mathrm{m}$. Teleoconch whorls weakly convex, suture deeply impressed. Axial sculpture of broad, prominent ribs extending from suture to suture ( 7 per whorl in holotype), including on last and penultimate whorls. Spiral sculpture of evenly spaced, strong, raised cords, nodulous at crossings with axial ribs, in holotype 2 on first whorl, 3 on other whorls, 7 on last whorl before transition to canal. Three or four weaker secondary cords situated between primary cords, slightly nodulous at intersections with raised growth lines. Two brown secondary cords situated in each interspace between primary cords on last whorl. Siphonal canal open, long, slightly turned to left and recurved, sculptured by 25 (in holotype) weaker cords of slightly uneven strength. Aperture with 8 weak lirae inside. Inner lip completely adherent to columella, forming very thin, shiny columellar callus with 4 columellar plicae, first very weak. No umbilicus. Protoconch transparent flesh colour, interior of aperture white. Teleoconch light tan with several brown spiral bands also present on canal. Major spiral cords slightly lighter than background, secondary spiral cords mostly brown.

Holotype measurements: SL $13.0 \mathrm{~mm}$, AL (without canal) $3.5 \mathrm{~mm}$, SW $4.7 \mathrm{~mm}$. 


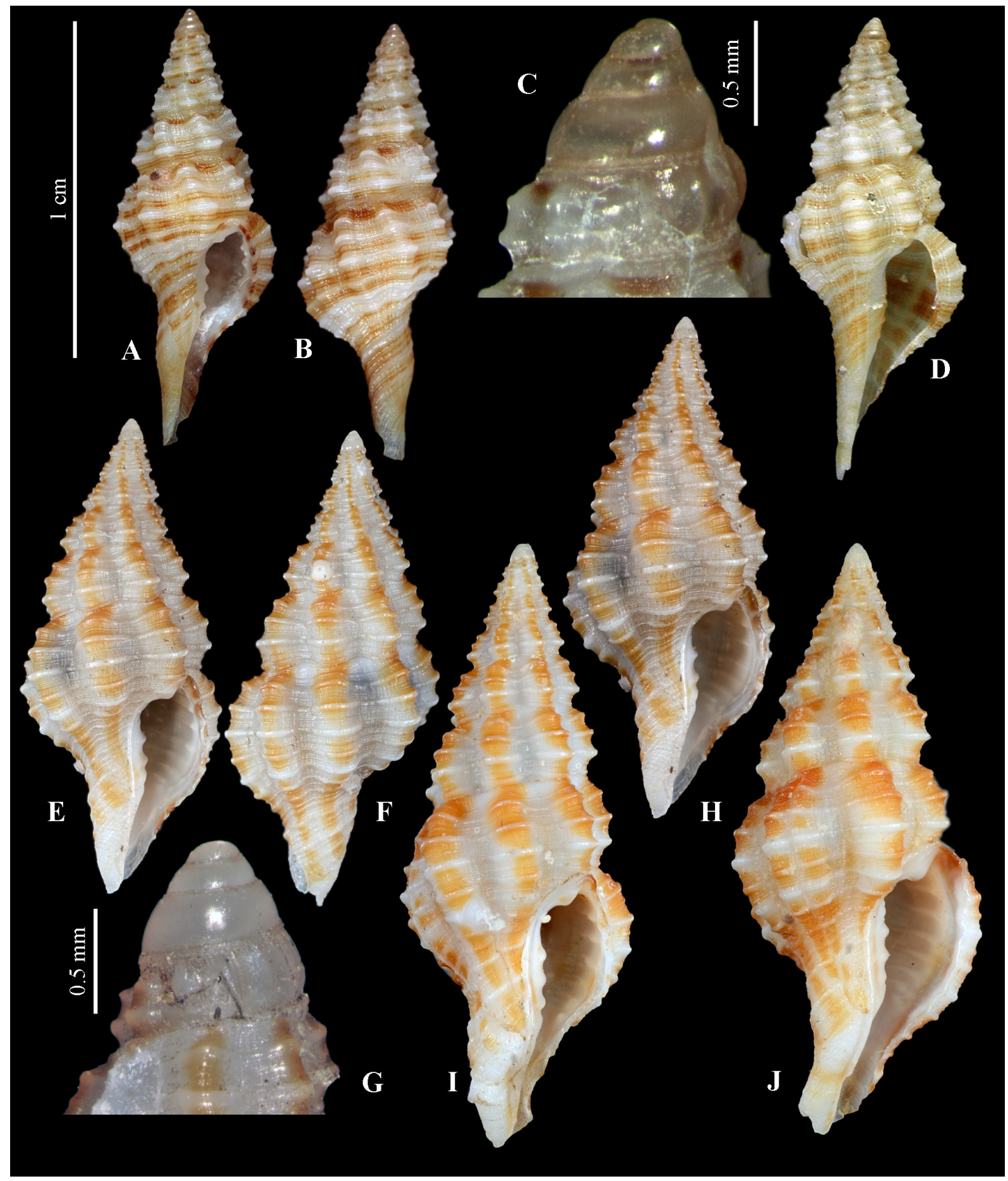

Fig. 7. A-D. Granulifusus norfolkensis sp. nov. A-C. Holotype, SL $13.0 \mathrm{~mm}$, sequenced (MNHN IM-2013-68811). D. New Caledonia, Norfolk Ridge, $23^{\circ} 23^{\prime} \mathrm{S}, 168^{\circ} 00^{\prime} \mathrm{E}, 430-480 \mathrm{~m}$, sequenced specimen (MNHN IM-2007-36921). E-J. Granulifusus tatianae sp. nov. E-G. Holotype (MNHN IM2000-33595). H. Paratype, SL 15.0 mm (MNHN IM-2000-33596). I. New Caledonia, SMIB 3, stn

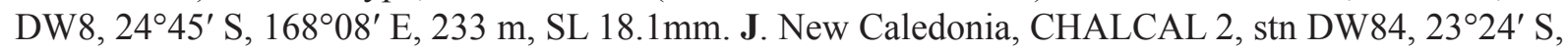
$168^{\circ} 07^{\prime} \mathrm{E}, 170 \mathrm{~m}$, SL $17.7 \mathrm{~mm}$. All shells to the same scale. 


\section{Distribution}

Known only from southern New Caledonia, off Ile des Pins and Norfolk Ridge, in 404-480 m. The multispiral protoconch suggests a planktonic dispersal phase and a possibly broader distribution.

\section{Remarks}

The species is known from just two specimens, the holotype and a slightly larger specimen attaining SL $13.8 \mathrm{~mm}$ (Fig. 7D). Granulifusus norfolkensis sp. nov. can be recognized by its small size, coloration and constricted aperture. Conchologically it is most similar to Granulifusus tatianae sp. nov., also from southern New Caledonia, but differs in having a smaller and more slender shell with longer siphonal canal and brown spiral bands.

\section{Granulifusus tatianae sp. nov. urn:lsid:zoobank.org:act:DBF2EAD7-3434-4F1F-A1A1-6ECE73BE5FC3}

Figs 4F, 7E-J

\section{Diagnosis}

Shell strong, broadly fusiform, up to $18 \mathrm{~mm}$, protoconch of 3.5 whorls. Teleoconch whorls strongly convex, axial sculpture of broad opisthocline axial ribs, spiral sculpture of strong primary cords and numerous finer secondary cords between them. Aperture lirate inside, columella with several distinct columellar plicae. Teleoconch light tan, axial ribs brown.

\section{Etymology}

The specific epithet is named in honour of Tatiana Steyker, wife of Yuri Kantor, patient permanent companion in all travels and many fieldtrips.

\section{Type material}

\section{Holotype}

NORFOLK RIDGE: south of New Caledonia, $23^{\circ} 17^{\prime} \mathrm{S}, 168^{\circ} 14^{\prime} \mathrm{E}, 190-212 \mathrm{~m}$, expedition NORFOLK 1 , stn DW1727 (MNHN IM-2000-33595).

\section{Paratype}

NORFOLK RIDGE: 1 specimen, same station as for holotype (MNHN IM-2000-33596); 1 specimen (ANSP, ex Snyder collection).

\section{Other material examined (all in MNHN)}

NEW CALEDONIA: BIOCAL: 1 dd, stn CP84, 2043' S, 16701' E, 150-210 m. - SMIB 3: 1 dd, stn

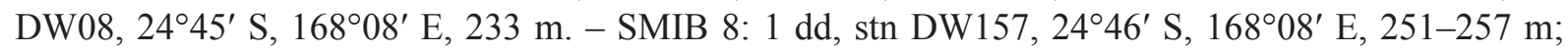
CHALCAL 2: 3 dd, stn DW84, 23⒉ $24^{\prime}$ S, $168^{\circ} 07^{\prime}$ E, 170 m. - NORFOLK 1: 1 lv, 2 dd; stn DW1724,

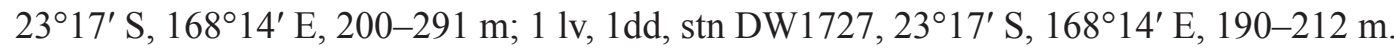

LOYALTY RIDGE: MUSORSTOM 6: 1 dd, stn DW442, 20 $54^{\prime} \mathrm{S}, 167^{\circ} 17^{\prime} \mathrm{E}, 200 \mathrm{~m} ; 1 \mathrm{lv}, 1 \mathrm{dd}, \operatorname{stn}$ DW473, $21^{\circ} 09^{\prime} \mathrm{S}, 167^{\circ} 55^{\prime} \mathrm{E}, 236 \mathrm{~m}$. - LIFOU 2000: 2 dd, stn DW1650, $20^{\circ} 54.2^{\prime} \mathrm{S}, 167^{\circ} 01.7^{\prime} \mathrm{E}, 120-250 \mathrm{~m}$.

WALLIS AND FUTUNA: MUSORSTOM 7: 1 dd, stn CP517, 14¹3’ S, 178¹0’ W, 233-235 m.

\section{Description}

Shell fusiform, solid, of 3.5 protoconch and 7 weakly convex teleoconch whorls in holotype. Protoconch bluntly conical (Fig. 7G), last $3 / 4$ whorl with axial riblets, protoconch/teleoconch discontinuity distinct, 
marked by appearance of teleoconch sculpture. Protoconch smooth except scattered microgranules, more dense along suture, where they are arranged in oblique lines. Protoconch diameter 820-910 $\mu \mathrm{m}$ (latter in holotype), exposed height 860-920 $\mu \mathrm{m}$ (former in holotype). Teleoconch whorls convex with impressed suture. Axial sculpture of broad, prominent axial ribs, aligned axially on spire from one whorl to the next, 7 ribs per whorl, and of very weak incremental lines. Spiral sculpture of evenly spaced, strong, raised primary cords, in holotype 3 on spire whorls, 13 on last whorl, and numerous, much weaker secondary threads, about 5-7 between two adjacent primary cords. Intersection of secondary spiral threads and incremental lines forming finely reticulate microsculpture. Siphonal canal open, short, recurved. Aperture with 8 strong lirae inside, adapicalmost stronger, forming spirally elongated tooth constricting base of siphonal canal. Inner lip partially detached, with three prominent columellar plicae and one strong parietal tooth, forming well-defined posterior canal. No umbilicus. Colour of protoconch and interior of aperture white. Teleoconch background colour creamy white, axial ribs light brown, spiral cords lighter than ribs.

Holotype measurements: SL $14.3 \mathrm{~mm}$, AL (without canal) $4.5 \mathrm{~mm}$, SW $6.1 \mathrm{~mm}$.

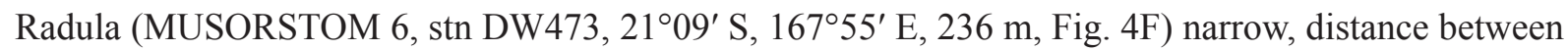
outer edges of lateral teeth about $55 \mu \mathrm{m}$, with slender central tooth, about twice as long as broad, bearing three strong cusps. Lateral tooth medium broad, with long basal 'handle' on outer edge, with five strong, subequal cusps, the outermost more clearly separated from the rest, and one smaller inner cusp. Narrow but distinct transverse folds of the membrane situated serially between rows of lateral teeth.

\section{Distribution}

Southwest Pacific, known only from Futuna and the New Caledonia region, alive in 200-236 m, empty shells in $170-251 \mathrm{~m}$.

\section{Remarks}

Granulifusus tatianae sp. nov. is superficially similar to Okutanius kuroseanus gen. et comb. nov. Both species have brown axial ribs aligned along the spire against a lighter, creamy background, and both have a multispiral protoconch indicating planktotrophic larval development. Okutanius kuroseanus gen. et comb. nov., however, reaches a much larger adult size and is proportionally broader at comparable size; it has a longer siphonal canal, weaker columellar plicae, and spiral cords of uneven strength, without the very regular pattern of evenly spaced primary cords separated by 5-7 very fine, even, spiral threads of G. tatianae sp. nov.

The species also resembles G. norfolkensis sp. nov. in sculpture and particularly in apertural armature. Nevertheless, the shell shape in Granulifusus tatianae sp. nov. is rather different, and the species can be easily distinguished by size at maturity: $G$. tatianae sp. nov. attains a significantly larger size, up to $18.1 \mathrm{~mm}$, versus $13.6 \mathrm{~mm}$ in G. norfolkensis sp. nov.

The new species was not examined molecularly and we tentatively assign it to Granulifusus based on the similarities of shell sculpture and apertural ornamentation to G. norfolkensis sp. nov. and G. williami comb. nov.

Granulifusus kurodai (Okutani \& Sakurai, 1964) comb. nov.

Fig. 8A-B

Pseudolatirus kurodai Okutani \& Sakurai, 1964: 58, pl. 2, fig. 4.

Pseudolatirus kurodai - Hasegawa \& Saito 1995: pl. 4, fig. 8 (paratype illustrated). 
Fusolatirus kurodai-Okutani 1975: 193, pl. 10, fig. 22; 2000: 507, pl. 252, fig. 12. - Higo, Callomon \& Gotō 2001: 87, fig. G3001 (holotype illustrated).

\section{Type material}

\section{Holotype}

JAPAN: off Gotō Islands, Nagasaki Prefecture, Western Kyūshū (NSMT Mo 38547), type locality corrected (Okutani \& Sakurai 1966) [not seen].

\section{Paratypes}

JAPAN: paratype 1, Hyotanse Bank (NSMT-Mo58524) (examined); paratype 2, off Gotō Islands (whereabouts unknown) (not seen); paratype 3, Kashiwajima (NSMT-Mo70270) (examined).

\section{Description}

Shell medium-sized, fusiform, with long, slightly sinuous siphonal canal. Protoconch paucispiral, domeshaped (Fig. 8B), of 1.75 whorls, with strongly convex first whorl and much flatter last $3 / 4$ of whorl, with two closely spaced and rather indistinct axial riblets at transition to teleoconch, protoconch/teleoconch discontinuity distinct. Protoconch diameter $900 \mu \mathrm{m}$, exposed height $750 \mu \mathrm{m}$. Teleoconch with regularly convex whorls, aperture with two very weak columellar plicae, and indistinct parietal tooth; spiral sculpture of unevenly spaced narrow cords of similar strength; axial ribs not aligned along the spire. Background colour cream to yellowish, primary spiral cords light tan.

\section{Distribution}

Known from Japan, in 100-200 m, records from other localities may refer to G. annae sp. nov. and G. guidoi sp. nov.

\section{Remarks}

We were able to study two paratypes NSMT-Mo58524 ( paratype 1) and NSMT-Mo70270 (paratype 3). They are clearly not conspecific, paratype 3 differing in its much larger protoconch and general shell shape and sculpture; it represents a juvenile Fusolatirus, resembling F. luteus Snyder \& Bouchet, 2006 (see Snyder \& Bouchet 2006: fig. 2 D-E). Conchologically, paratype 1 is very similar to the holotype and is the largest known specimen (SL $35.1 \mathrm{~mm}$ ).

Conchologically Granulifusus kurodai comb. nov. is similar to G. annae sp. nov.; for differences see the description of the latter. In the absence of data on radula and molecular phylogeny, the assignment of this species to Granulifusus is tentative, based on its shell resemblance to sequenced species such as G. annae sp. nov.

\section{Granulifusus guidoi sp. nov. urn:1sid:zoobank.org:act:8630D56D-6613-46EA-BE2D-3F0F4020FC1C}

Fig. 8E-H

Latirus kurodai - Mallard \& Robin 2005: pl. 45, lower left image (not of Okutani \& Sakurai 1964). Poppe 2008: pl. 348, figs 1-2 (not of Okutani \& Sakurai 1964).

Pseudolatirus kurodai - Mallard \& Robin 2017: 153 (not of Okutani \& Sakurai 1964).

\section{Diagnosis}

Shell strong, slender, fusiform, height up to $31 \mathrm{~mm}$, protoconch of 2.5 whorls. Teleoconch whorls strongly convex, axial sculpture of medium broad opisthocline axial ribs, spiral sculpture of thin, 
distinct, primary cords, varying slightly in size. Aperture lirate inside, columella with distinct to weak columellar plicae. Teleoconch light tan with light orange-brown canal and caramel-colored spiral cords.

\section{Etymology}

Named for Guido Poppe, the author of the landmark Philippine Marine Molluscs and discoverer of many new molluscs in the Philippines and elsewhere, who generously provided some of the type material.

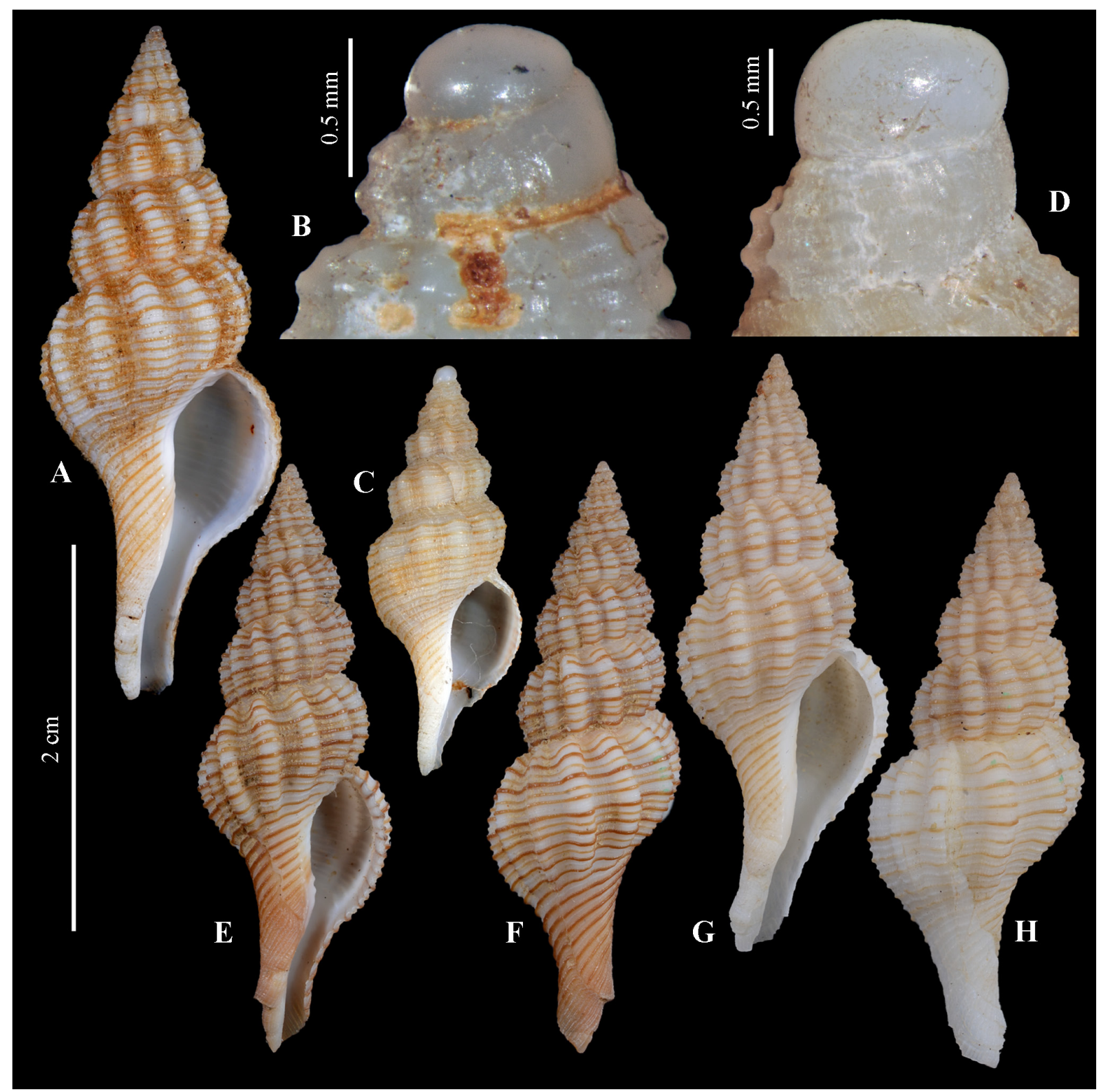

Fig. 8. A-B. Granulifusus kurodai (Okutani \& Sakurai, 1964) comb. nov., paratype 1, SL $35.1 \mathrm{~mm}$ (NSMT-Mo58524). C-D. Fusolatirus sp. aff. luteus Snyder \& Bouchet, 2006 (paratype 3 of Pseudolatirus kurodai, see the text), SL $21.5 \mathrm{~mm}$ (NSMT-Mo70270). E-H. Granulifusus guidoi sp. nov. E-F. Holotype, SL 30.2 mm (MNHN IM-2000-33597). G-H. Paratype, SL 30.7 mm (MNHN IM-200033598). All shells at the same scale. 


\section{Type material}

\section{Holotype}

PHILIPPINES: off Aliguay Island, northeast of Mindanao, in 50-150 m (MNHN IM-2000-33597, ex Snyder collection).

\section{Paratypes}

PHILIPPINES: 1 specimen, same data as for holotype, SL 30.7 mm (MNHN-2000-33598); 1 specimen, trawled off Aliguay Island, 80-150 m, SL 30.7 mm (ANSP 456853); 1 specimen, trawled off Aliguay Island, 100-150 m, $25.5 \mathrm{~mm}$ (NMP).

\section{Description}

Shell slender, fusiform, of average size for genus. Protoconch multispiral, of 2.5 smooth whorls in holotype, with 4 axial riblets on last whorl, pale grey-orange. Transition to teleoconch marked by groove between last riblet and first teleoconch axial rib. Teleoconch of 8-9 convex whorls, suture deep, adpressed. Axial sculpture of distinct ribs, closely spaced, 10 on earlier whorls, increasing to 12 on later whorls. Spiral sculpture of dark cords, often with less prominent and paler secondary cords between them. First whorl with single dark cord, adding roughly one cord per whorl, with 7 cords on penultimate whorl. Cords continue to anterior end of canal, crowding closer together at tip; in holotype, last whorl with about 25 cords. Siphonal canal recurved back from aperture, moderately long, open. Dark spiral cords matched on inner lip by low, light brown lirae. Columellar callus white, with a few spiral lines continuing inward at adapical end of shield. Two stronger rounded columellar plicae situated towards aperture and one much weaker towards canal. Teleoconch white to light tan with light orange-brown neck; caramel-colored spiral cords on entire shell.

Holotype measurements: SL $30.2 \mathrm{~mm}, \mathrm{AL}$ (without canal) $8.1 \mathrm{~mm}$, SW $10.1 \mathrm{~mm}$.

\section{Distribution}

Known only from the type locality.

\section{Remarks}

Granulifusus guidoi sp. nov. is most similar to G. kurodai comb. nov., with which it is often confused. It differs by a multispiral protoconch with more whorls, and a narrower shell with narrower aperture. It is also similar to Okutanius ellenae gen. et sp. nov., which has white cords with dark interspaces on the teleoconch whereas G. guidoi sp. nov. has dark, caramel-colored cords with light tan interspaces.

In the absence of data on radula and molecular phylogeny, we tentatively assign this species to Granulifusus based on shell similarities to such sequenced species as G. annae sp. nov.

$$
\begin{aligned}
& \text { Okutanius gen. nov. } \\
& \text { urn:Isid:zoobank.org:act:FAE994AB-DCBE-4436-BBAD-2D1283674F12 }
\end{aligned}
$$

\section{Type species}

Fusolatirus kuroseanus Okutani, 1975, here designated.

\section{Diagnosis}

Shell medium-size, reaching $30 \mathrm{~mm}$, fusiform to broad fusiform, strong, with spiral colour bands. Teleoconch whorls evenly strongly convex, shell base sharply narrowing towards long, weakly sinuous siphonal canal. Protoconch multispiral, of 3-3.75 whorls. Axial sculpture of distinct broad, weakly 
opisthocline, closely spaced ribs. Spiral sculpture of distinct primary cords, subequal in size, and much thinner cordlets between them. Aperture narrow, oval, relatively small, columella with several weak plicae. No distinct parietal knob.

Operculum spans entire aperture, with terminal nucleus.

Lateral teeth with five closely spaced, slightly curved cusps and much smaller, but distinct, innermost cusp. Outermost very short small cusp present, but usually overlaid by large next cusp and not seen in dorsal view. Central tooth small, narrow, trapezoid, with three cusps. Narrow but distinct transverse folds of membrane situated serially between rows of lateral teeth.

\section{Etymology}

The genus is named after Takashi Okutani in recognition of his lifelong contribution to malacology, including the description of the type species of the genus. The gender is masculine.

\section{Differential diagnosis}

Okutanius gen. nov. is very similar to Granulifusus and conchological and morphological characters overlap at least in some species. Species of Okutanius gen. nov. do not reach as large size as in Granulifusus. Nevertheless, without molecular data, it would be difficult to suggest the existence of two clearly separate genera.

\section{Genus composition}

In addition to two sequenced species, $O$. kuroseanus and $O$. ellenae sp. nov., we allocate Pseudolatirus aikeni Lussi, 2014, known from empty shell only, to Okutanius gen. nov. based on shell similarity to sequenced $O$. kuroseanus.

Okutanius kuroseanus (Okutani, 1975) gen. et comb. nov.

Figs 4G, 9A-G

Fusolatirus kuroseanus Okutani, 1975: 193, pl. 10, fig 21.

Pseudolatirus leucostriatus Kosuge, 1979: 31, pl. 5, fig. 5, syn. nov.

Fusolatirus kuroseanus - Okutani 2000: 507, pl. 252, fig. 11. - Marais \& Kilburn 2010: 127.

Latirus kuroseanus - Mallard \& Robin 2005: caption on pl. 45.

Pseudolatirus kuroseanus - Mallard \& Robin 2017: 153.

\section{Type material}

JAPAN: holotype of of F. kuroseanus, Kurose Bank, off Izu islands, south of Honshū, $33^{\circ} 23.5^{\prime} \mathrm{N}$, 139 36. $2^{\prime}$ E, 260 m (NSMT Mo 69575) (illustrated by Higo et al. 2001: 87); holotype of P. leucostriatus (said to be off Midway Island, $32^{\circ} 02^{\prime} \mathrm{N}, 173^{\circ} 06^{\prime} \mathrm{W}, 365 \mathrm{~m}$, but label of holotype says $29^{\circ} 46.4^{\prime} \mathrm{N}$, $179^{\circ} 02.9^{\prime} \mathrm{E}, 265 \mathrm{~m}$ ) in the Institute of Malacology, Tokyo (IMT 79-18) [examined]; paratype, same location as holotype, said to be donated to USNM.

\section{Sequenced material}

PAPUA NEW GUINEA, NEW IRELAND: KAVIENG 2014: 1 lv, stn CP4503, 02³0' S, $150^{\circ} 44^{\prime}$ E, 191-200 m (MNHN IM-2013-59070). 


\section{Other material examined}

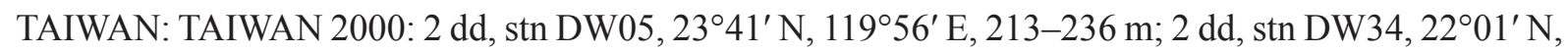
$120^{\circ} 36^{\prime}$ E, 240-246 m; 25 dd, stn DW36, 21 $1^{\circ} 54^{\prime} \mathrm{N}, 120^{\circ} 36^{\prime} \mathrm{E}, 300-331 \mathrm{~m}$.

PHILIPPINES: 2 spms, Panglao, Bohol, tangle nets, 160 m, (ANSP 425326); 2 spms, off Aliguay I., Mindanao, $150 \mathrm{~m}$ (ANSP 425322); $1 \mathrm{spm}$, Bohol, Balicasag, $240 \mathrm{~m}$ (ANSP 425324); $1 \mathrm{spm}$, Philippine Is, tangle nets (ANSP 425325); 1 spm, Balicasag I., Bohol, tangle nets, 240 m (ANSP 425327); 1 spm, Siquijor I., tangle nets, $150 \mathrm{~m}$ (ANSP 425323).

ARAFURA SEA: KARUBAR: 1 lv, $13 \mathrm{dd}$, stn DW15, 05²17' S, 132 $41^{\prime} \mathrm{E}, 212-221 \mathrm{~m}$.

SOLOMON ISLANDS: SALOMON 1: $1 \mathrm{dd}$, stn DW1765, 08²43' S, $160^{\circ} 07^{\prime}$ E, 325-380 m; 1 dd, stn DW1812, $09^{\circ} 46^{\prime} \mathrm{S}, 160^{\circ} 52^{\prime} \mathrm{E}, 222-246 \mathrm{~m}$.

NEW CALEDONIA: “Vauban” 1978-79: 1 dd, stn DR37, 22³2' S, 166²6' E, 175-250 m. - BIOCAL: $1 \mathrm{dd}$, stn DW81, $20^{\circ} 29^{\prime} \mathrm{S}, 166^{\circ} 47^{\prime} \mathrm{E}, 430-470 \mathrm{~m}$; 1 juv. Lv, stn CP105, $21^{\circ} 31^{\prime} \mathrm{S}, 166^{\circ} 22^{\prime} \mathrm{E}, 330-335 \mathrm{~m}$.

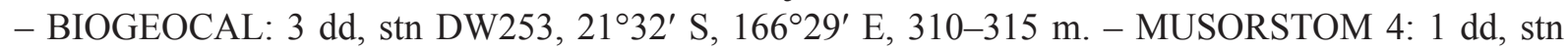

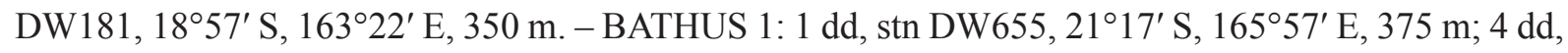

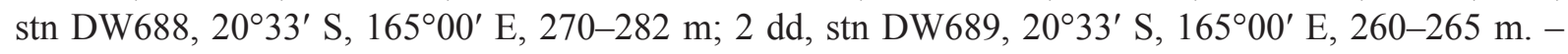
BATHUS 4: $1 \mathrm{dd}, \operatorname{stn}$ DW886, $21^{\circ} 01^{\prime} \mathrm{S}, 164^{\circ} 28^{\prime} \mathrm{E}, 250-300 \mathrm{~m}$; $1 \mathrm{dd}, \operatorname{stn} \mathrm{DW} 887,21^{\circ} 07^{\prime} \mathrm{S}, 164^{\circ} 28^{\prime} \mathrm{E}$, 320-344 m. - SMIB 4: 1 dd, stn DW44, 244' S, 168 08' E, 270-300 m. - SMIB 5: 1 dd, stn DW90, $22^{\circ} 19^{\prime} \mathrm{S}, 168^{\circ} 42^{\prime}$ E, 340 m. - SMIB 8: 1 dd, stn DW160, 244ㄱ' S, 168 $08^{\prime}$ E, 280-282 m.

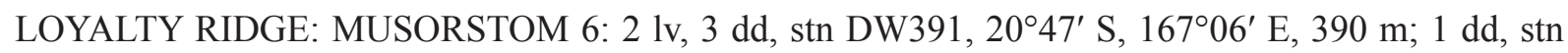

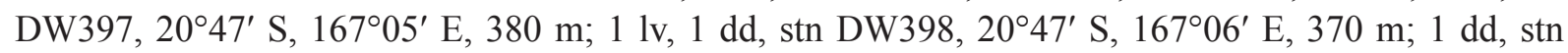
DW418, 20 $42^{\prime} \mathrm{S}, 167^{\circ} 03^{\prime} \mathrm{E}, 283 \mathrm{~m} ; 3 \mathrm{dd}$, stn DW459, $21^{\circ} 01^{\prime} \mathrm{S}, 167^{\circ} 31^{\prime} \mathrm{E}, 425 \mathrm{~m} ; 3 \mathrm{dd}$, stn DW480,

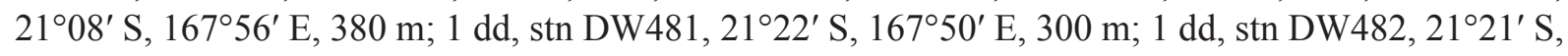
$167^{\circ} 47^{\prime}$ E, $375 \mathrm{~m} ; 2 \mathrm{dd}$, stn DW487, 21 ${ }^{\circ} 23^{\prime} \mathrm{S}, 167^{\circ} 46^{\prime} \mathrm{E}, 500 \mathrm{~m}$.

VANUATU: MUSORSTOM 8: 1 dd, stn DW1059, 16²13' S, 167²1' E, 408-430 m; 1 dd, stn DW1065, $16^{\circ} 16^{\prime} \mathrm{S}, 167^{\circ} 21^{\prime} \mathrm{E}, 360-419 \mathrm{~m}$.

WALLIS AND FUTUNA: MUSORSTOM 7: 1 dd, stn DW496, $14^{\circ} 20^{\prime} \mathrm{S}, 178^{\circ} 04^{\prime} \mathrm{W}, 250-330 \mathrm{~m}$

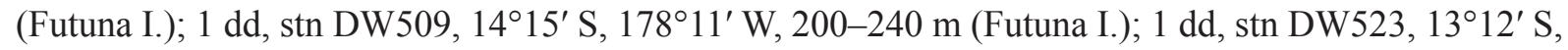
$176^{\circ} 16^{\prime} \mathrm{W}, 455-515 \mathrm{~m}$ (Wallis I.); $1 \mathrm{dd}$, stn DW569, 12 $2^{\circ} 30^{\prime} \mathrm{S}, 176^{\circ} 51^{\prime} \mathrm{W}, 300-305 \mathrm{~m}$ (Waterwitch Bank); 1 dd, stn DW571, $12^{\circ} 31^{\prime} \mathrm{S}, 176^{\circ} 52^{\prime} \mathrm{W}, 502-508 \mathrm{~m}$ (Waterwitch Bank); 1 dd, stn DW610, $13^{\circ} 21^{\prime} \mathrm{S}, 176^{\circ} 09^{\prime} \mathrm{W}, 286 \mathrm{~m}$ (Wallis I.); 1 dd, stn DW626, $11^{\circ} 54^{\prime} \mathrm{S}, 179^{\circ} 32^{\prime} \mathrm{W}, 597-600 \mathrm{~m}$ (Bayonnaise Bank).

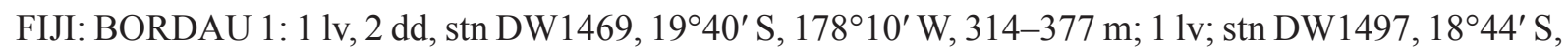
$178^{\circ} 25^{\prime} \mathrm{W}, 335-350 \mathrm{~m}$.

TONGA: BORDAU 2: 2 dd, stn DW1535, 21 ${ }^{\circ} 43^{\prime} \mathrm{S}, 175^{\circ} 18^{\prime} \mathrm{W}, 268 \mathrm{~m} ; 3 \mathrm{dd}, \operatorname{stn} \mathrm{DW} 1567,2^{\circ} 02^{\prime} \mathrm{S}$,

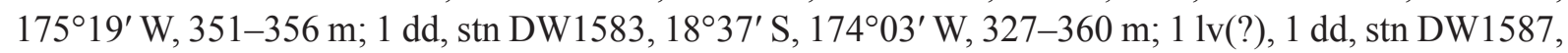

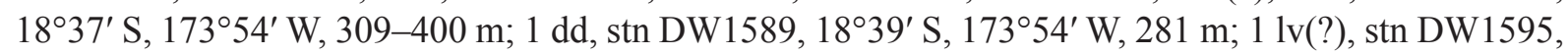

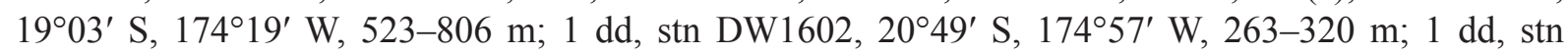
DW1605, $22^{\circ} 17^{\prime} \mathrm{S}, 175^{\circ} 16^{\prime} \mathrm{W}, 441 \mathrm{~m}$; 1 lv, stn DW1607, $22^{\circ} 15^{\prime} \mathrm{S}, 175^{\circ} 23^{\prime} \mathrm{W}, 356-367 \mathrm{~m}$; 1 lv(?), stn

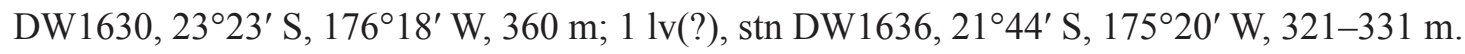

CHRISTMAS ISLAND: 2 dd, off NE point, $10^{\circ} 25^{\prime} \mathrm{S}, 105^{\circ} 42^{\prime} \mathrm{E}, 183 \mathrm{~m}$, coll. R. Kirkpatrick 1916 (AMS C324273). 


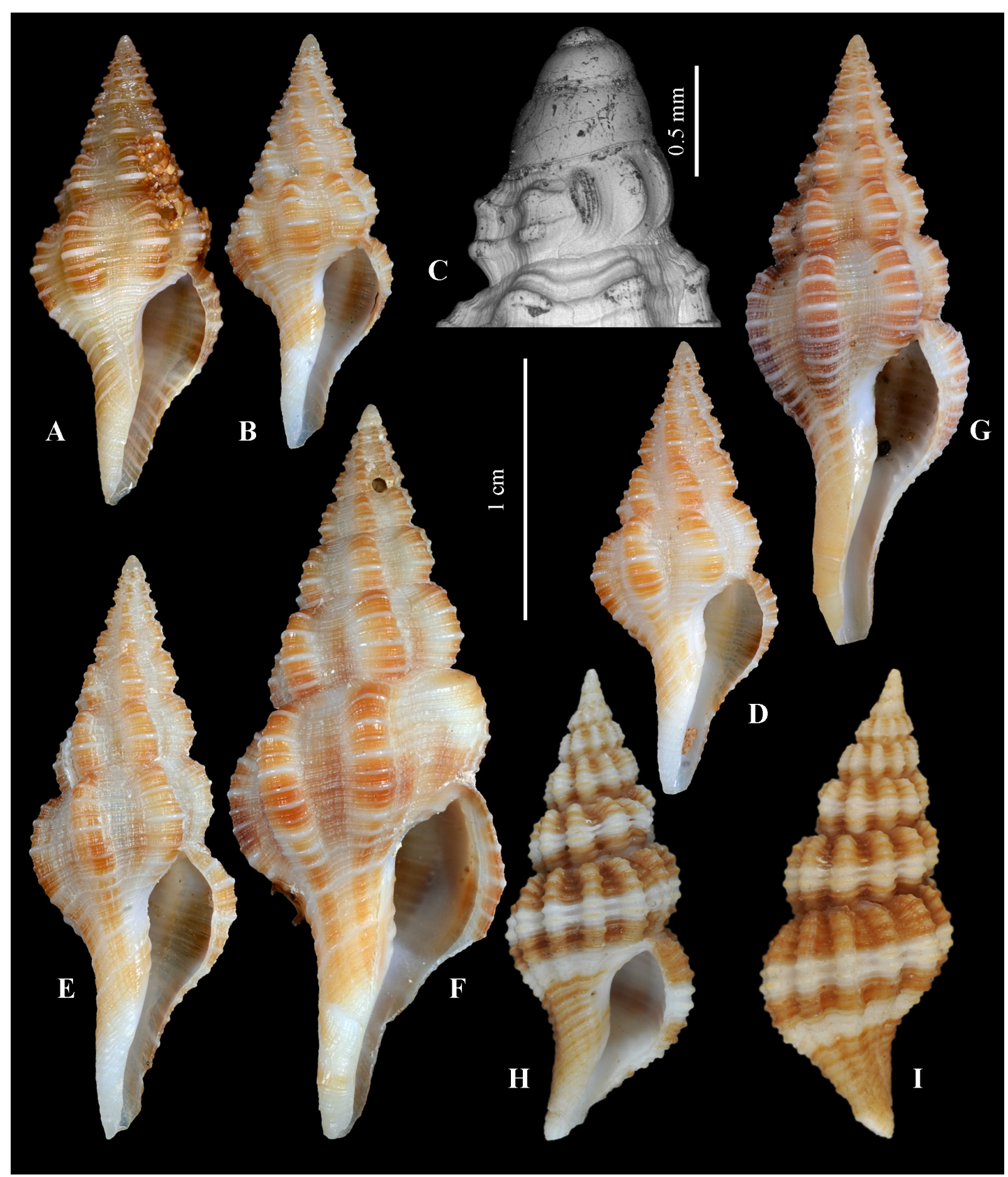

Fig. 9. A-G. Okutanius kuroseanus (Okutani, 1975) gen. et comb. nov. A. New Ireland, KAVIENG 2014, stn CP4503, $02^{\circ} 30^{\prime} \mathrm{S}, 150^{\circ} 44^{\prime} \mathrm{E}, 191-200 \mathrm{~m}$, SL $18.1 \mathrm{~mm}$, sequenced specimen; for radula, see Fig. 4G (MNHN IM-2013-59070). B. BORDAU 1, stn DW1469, 1940' S, 178 10' W, 314-377 m, SL 15.9 mm. C. Protoconch, New Caledonia, BIOCAL, stn CP105, 21 ${ }^{\circ} 31^{\prime} \mathrm{S}, 166^{\circ} 22^{\prime} \mathrm{E}, 330-335 \mathrm{~m}$. D-E. Loyalty Ridge, MUSORSTOM 6, stn DW391, 2047' S, $167^{\circ} 06^{\prime}$ E, 390 m. D. SL 17.3 mm. E. SL

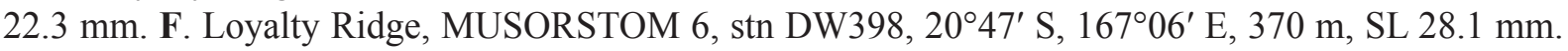
G. Taiwan. TAIWAN 2000, stn DW34, 22 ${ }^{\circ} 2^{\prime} \mathrm{N}, 120^{\circ} 36^{\prime} \mathrm{E}, 246 \mathrm{~m}$, SL $23.4 \mathrm{~mm}$. H-I. Okutanius aikeni (Lussi, 2014) gen. et comb. nov., holotype, SL $25.6 \mathrm{~mm}$. A-B, D-G at the same scale, H-I not to scale. 
RÉUNION: MD32: 1 dd, stn CP129, 2051' S, 55³6' E, 290-300 m; 1 dd worn, stn DC136, 2046’ S, $55^{\circ} 36^{\prime}$ E, 915-922 m.

SOUTH AFRICA: $1 \mathrm{dd}$, former Transkei, off Port Grosvenor, 295․ $4^{\prime} \mathrm{S}, 31^{\circ} 26.4^{\prime} \mathrm{E}, 105 \mathrm{~m}$ (NM C636); 1 lv(?), former Transkei, off Mtamvuna River, 31 ${ }^{\circ} 08.4^{\prime} \mathrm{S}, 30^{\circ} 16.6^{\prime} \mathrm{E}, 160 \mathrm{~m}$ (NM C9652); $2 \mathrm{dd}$, Zululand, SE of Port Durnford, 2905.8' S, 32 09.4' E, 165 m (NM S903); 1 dd, Zululand, SE of Port Durnford, 2904.6' S, 3209.0' E, 142 m (NM D7822).

\section{Description}

Shell fusiform, up to $28 \mathrm{~mm}$ in length. Protoconch acutely conical, multispiral, of 3 smooth, weakly convex whorls, with several slightly arcuate axial riblets on last $1 / 2$ whorl (Fig. 9C). Protoconch diameter $870 \mu \mathrm{m}$, exposed height $1000 \mu \mathrm{m}$. Teleoconch with regularly convex whorls, without imbricated subsutural spiral ridge, with long siphonal canal; aperture with weak columellar plication, and indistinct parietal tooth; spiral sculpture of unevenly arranged cords of uneven strength; axial ribs aligned along the spire. Background colour from creamy light to yellowish brown, axial ribs brown, primary spiral cords white.

Radula (Fig. 4G) examined in two specimens, from Fiji (BORDAU 1, stn DW1497) and from the New Ireland (MNHN IM-2013-59070, $18.1 \mathrm{~mm}$ ) (shell: Fig. 9A). They are very similar and that from the New Ireland specimen is illustrated and described. Radula narrow, distance between outer edges of lateral teeth about $70 \mu \mathrm{m}$ (1.34\% of AL). Lateral teeth broad, slightly arcuate, with strongly attenuated outer anterior corner, producing long 'handle'. Lateral teeth with outermost small cusp, on most rows overlaid by following major cusp and not seen on ventral view (marked by white arrow), five almost equal, closely spaced, cusps, outer one more clearly separated from the rest, and one smaller inner cusp. Central tooth trapezoid, with three short blunt cusps, central slightly longer. Distinct transverse folds of the membrane situated serially between rows of lateral teeth, reaching anterior margin of central tooth.

\section{Distribution}

Southwest Pacific Ocean, Indian Ocean to KwaZulu-Natal, in 150-620 m, alive in 150-400 m.

\section{Remarks}

This distinct, medium-sized species is similar to Granulifusus tatianae sp. nov.; for differences, see the description of the latter. It also resembles Okutanius ellenae gen. et sp. nov., but can be easily differentiated from the latter species by the colour pattern.

Okutanius ellenae gen. et sp. nov. urn:1sid:zoobank.org:act:BF7E85A5-AC8A-4A6C-A7BA-671948DB2EE1

Figs $4 \mathrm{H}, 10$

\section{Diagnosis}

Shell strong, slender, narrowly fusiform, up to $30 \mathrm{~mm}$, protoconch of 3.75 whorls. Teleoconch whorls strongly convex, axial sculpture of broad, nearly orthocline, axial ribs, spiral sculpture of dense, evenly spaced, strong, raised cords. Aperture lirate inside, columella with weak columellar plicae to nearly smooth. Teleoconch with alternating white spiral cords and orange brown interspaces, and three white bands, one subsutural, one just below periphery of last whorl and one at tip of siphonal canal.

\section{Molecular diagnosis}

See Table 3. 
Table 3. Molecular diagnosis of the Okutanius ellenae gen. et sp. nov.

\begin{tabular}{|c|c|}
\hline $\begin{array}{l}\text { Sequences analyzed } \\
\text { Independent combinations }\end{array}$ & 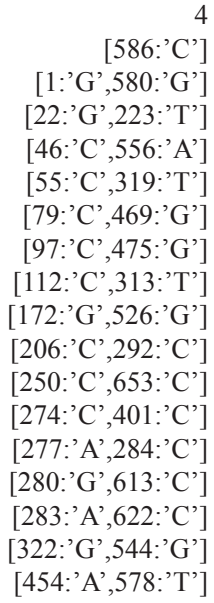 \\
\hline
\end{tabular}

\section{Etymology}

The specific epithet pays homage to Ellen Strong, in fitting tribute for this elegant and handsome species.

\section{Type material}

\section{Holotype}

NEW CALEDONIA: South of Ile des Pins, $22^{\circ} 53^{\prime}$ S, $167^{\circ} 35^{\prime}$ E, 376-390 m, expedition KANACONO, stn DW4677, sequenced (MNHN IM-2013-68819).

\section{Paratypes}

NEW CALEDONIA: 1 sequenced specimen from the type locality (MNHN IM-2013-68876); 2 specimens, $24^{\circ} 44^{\prime} \mathrm{S}, 168^{\circ} 10^{\prime} \mathrm{E}, 270-350 \mathrm{~m}$, expedition BERYX 11, stn DW11 (AMS C.572407 and NSMT-Mo 79000); 1 specimen, $22^{\circ} 58^{\prime} \mathrm{S}, 167^{\circ} 33^{\prime} \mathrm{E}, 410-440 \mathrm{~m}$, expedition MUSORSTOM 4, stn DW222 (ANSP 476089).

\section{Sequenced material}

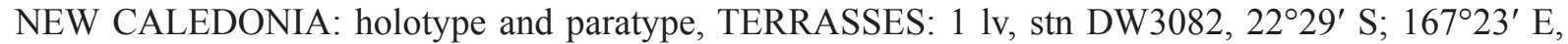
290 m (MNHN IM-2007-38356).

CORAL SEA: EBISCO: 1 lv, stn DW2520, Banc Kelso, 240' S, 159 41' E, 350-400 m (MNHN IM2007-32522).

\section{Other material examined}

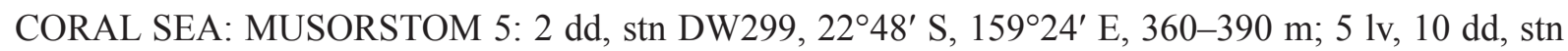

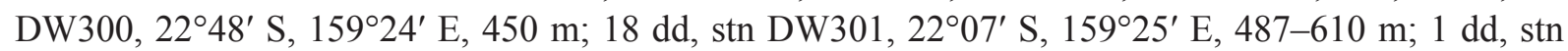
DW304, $22^{\circ} 10^{\prime} \mathrm{S}, 159^{\circ} 26^{\prime} \mathrm{E}, 385-420 \mathrm{~m} ; 2$ dd, stn DW306, $22^{\circ} 08^{\prime} \mathrm{S}, 159^{\circ} 21^{\prime} \mathrm{E}, 375-415 \mathrm{~m} ; 1$ lv, $3 \mathrm{dd}$, stn DC362, $19^{\circ} 53^{\prime} \mathrm{S}, 158^{\circ} 40^{\prime} \mathrm{E}, 410 \mathrm{~m}$.

NEW CALEDONIA: LAGON: 1 lv, stn 444, $18^{\circ} 15^{\prime} \mathrm{S}, 162^{\circ} 59^{\prime} \mathrm{E}, 300-350 \mathrm{~m}$. - BIOCAL: $1 \mathrm{dd}$, stn DW38, $23^{\circ} 00^{\prime} \mathrm{S}, 167^{\circ} 15^{\prime} \mathrm{E}, 360 \mathrm{~m} ; 8 \mathrm{lv}+\mathrm{dd}$, stn DW64, 244ㅇ $48^{\prime} \mathrm{S}, 168^{\circ} 09^{\prime} \mathrm{E}, 250 \mathrm{~m} ; 1 \mathrm{lv}, 3 \mathrm{dd}$ stn

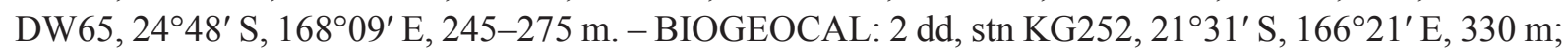

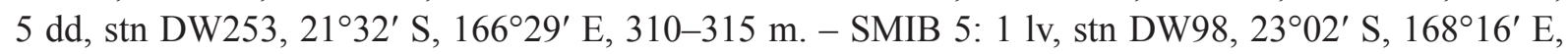

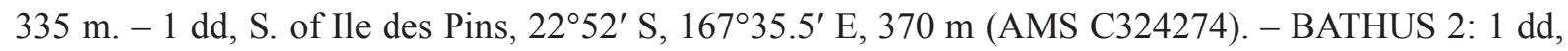

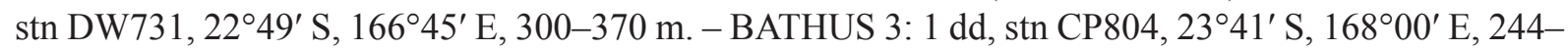


278 m. - MUSORSTOM 4: 1 lv, stn DW222, 22 ${ }^{\circ} 58^{\prime}$ S, 167³3' E, 410-440 m (paratype ANSP); 2 dd,

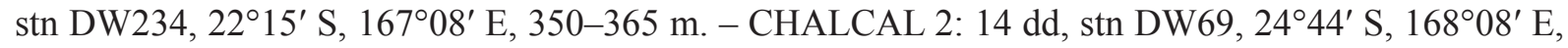

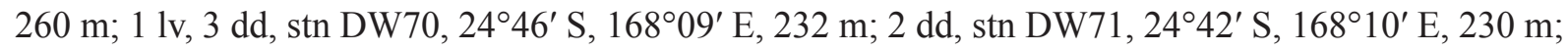
$1 \mathrm{dd}, \operatorname{stn}$ DW78, $23^{\circ} 41^{\prime} \mathrm{S}, 168^{\circ} 00^{\prime} \mathrm{E}, 233 \mathrm{~m} ; 3 \mathrm{dd}$, stn DW79, $23^{\circ} 41^{\prime} \mathrm{S}, 168^{\circ} 00^{\prime} \mathrm{E}, 243 \mathrm{~m}, 3 \mathrm{dd}$. - BERYX

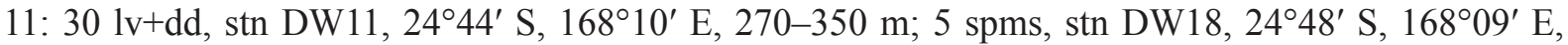
250-270 m. - HALICAL 1: $1 \mathrm{dd}$, stn 1-4, $18^{\circ} 53^{\prime}-18^{\circ} 56^{\prime} \mathrm{S}, 1^{\circ} 3^{\circ} 24^{\prime} \mathrm{E}, 350-400 \mathrm{~m}$. - LITHIST: $1 \mathrm{dd}$,

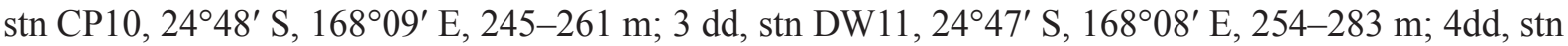

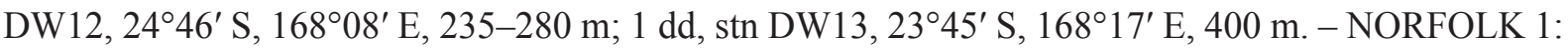

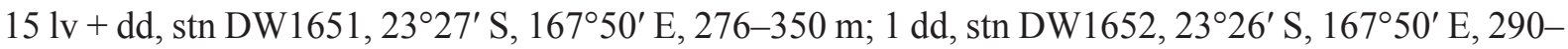
$378 \mathrm{~m}$; 3 dd, stn DW1653, $23^{\circ} 28^{\prime} \mathrm{S}, 167^{\circ} 51^{\prime} \mathrm{E}, 328-340 \mathrm{~m}$; 1 dd, stn DW1654, $23^{\circ} 28^{\prime} \mathrm{S}, 1^{\prime 6} 7^{\circ} 52^{\prime} \mathrm{E}$,

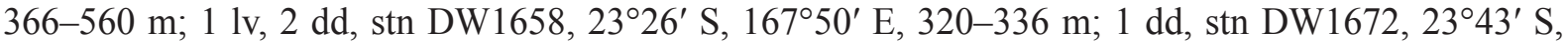

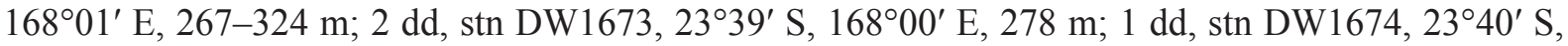

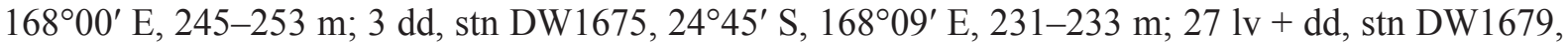

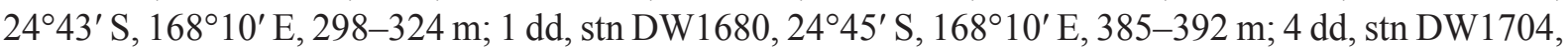

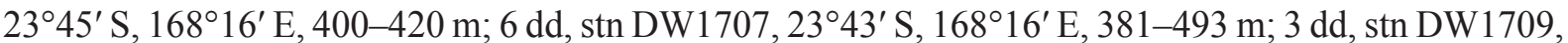

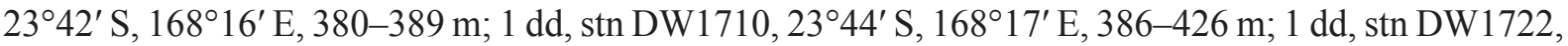

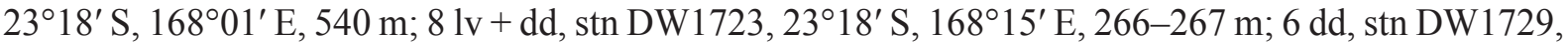

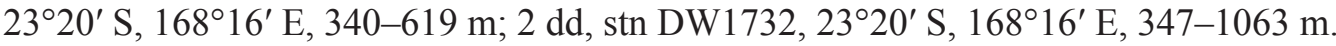

VANUATU: VOLSMAR: $2 \mathrm{dd}$, stn DW07, $22^{\circ} 26^{\prime} \mathrm{S}, 171^{\circ} 44^{\prime} \mathrm{E}, 325-400 \mathrm{~m} ; 1 \mathrm{dd}, \operatorname{stn} \mathrm{DW} 16,22^{\circ} 25^{\prime} \mathrm{S}$, $171^{\circ} 41^{\prime}$ E, 420-500 m; 1 dd, stn DW39, $22^{\circ} 20^{\prime}$ S, $168^{\circ} 44^{\prime}$ E, 280-305 m; 1 dd, stn DW49, $21^{\circ} 00^{\prime} \mathrm{S}$, $170^{\circ} 04^{\prime}$ E, $285 \mathrm{~m}$; 1 dd, stn DW50, 205' S, 170 04' E, 425 m. - MUSORSTOM 8: 1 dd, stn DW967, $20^{\circ} 19^{\prime} \mathrm{S}, 169^{\circ} 53^{\prime} \mathrm{E}, 295-334 \mathrm{~m}$.

TONGA: BORDAU 2: 2 dd, stn DW1607, $22^{\circ} 15^{\prime} \mathrm{S}, 175^{\circ} 23^{\prime} \mathrm{W}, 356-367 \mathrm{~m}$.

\section{Description}

Shell thin, slender, fusiform, consisting in holotype of 3.75 protoconch and 7 teleoconch whorls. Protoconch conical (Fig. 10C), with weakly convex whorls, last $1 \frac{1}{2}$ whorl with 6 axial riblets, protoconch/ teleoconch discontinuity distinct. Protoconch diameter $1070 \mu \mathrm{m}$, exposed height $1110 \mu \mathrm{m}$. Teleoconch whorls convex, suture deep, adpressed. Axial sculpture of broad, prominent ribs, aligned axially on spire from one whorl to the next, 6 ribs per whorl. Spiral sculpture of evenly spaced, strong, raised cords, in holotype 2 on first whorl, 3 on subsequent whorls, abapicalmost stronger, 5 on antepenultimate and penultimate whorls, 22 on last whorl. No second order axial or spiral sculpture. Siphonal canal open, long, slightly recurved. Aperture with 11 weak lirae inside, base of siphonal canal not clearly demarcated. Inner lip completely adherent to columella, forming smooth, white, shiny columellar shield, with three weak columellar plicae. No umbilicus. Colour of protoconch and interior of aperture white or fleshcoloured. Teleoconch with alternating white spiral cords and orange brown interspaces, and three white bands, one subsutural, one just below periphery of last whorl and one at tip of siphonal canal.

Holotype measurements: SL 22.7, LWL 15.4 mm, AL (without canal) 5.7 mm, SW 7.8 mm.

Live animal has orange body (Fig. 10D) with irregular white spots, more numerous on body than on foot. Operculum transparent, yellow, with terminal nucleus shifted leftwards, closing aperture completely.

Radula (Fig. 4H) examined in two specimens from Norfolk Ridge (NORFOLK 1, stn DW1658, and stn DW1679), very similar in both specimens. Radula narrow, distance between outer edges of lateral teeth about $80 \mu \mathrm{m}$ (1.6\% of AL). Lateral teeth medium broad, arcuate, with attenuated outer anterior corner, producing medium long 'handle'. Lateral teeth with outermost small cusp, on most rows overlaid by following major cusp and not seen on ventral view (marked by white arrow), five slightly recurved, short cusps, subequal in length, outer more distant from others, and one additional innermost short, but 


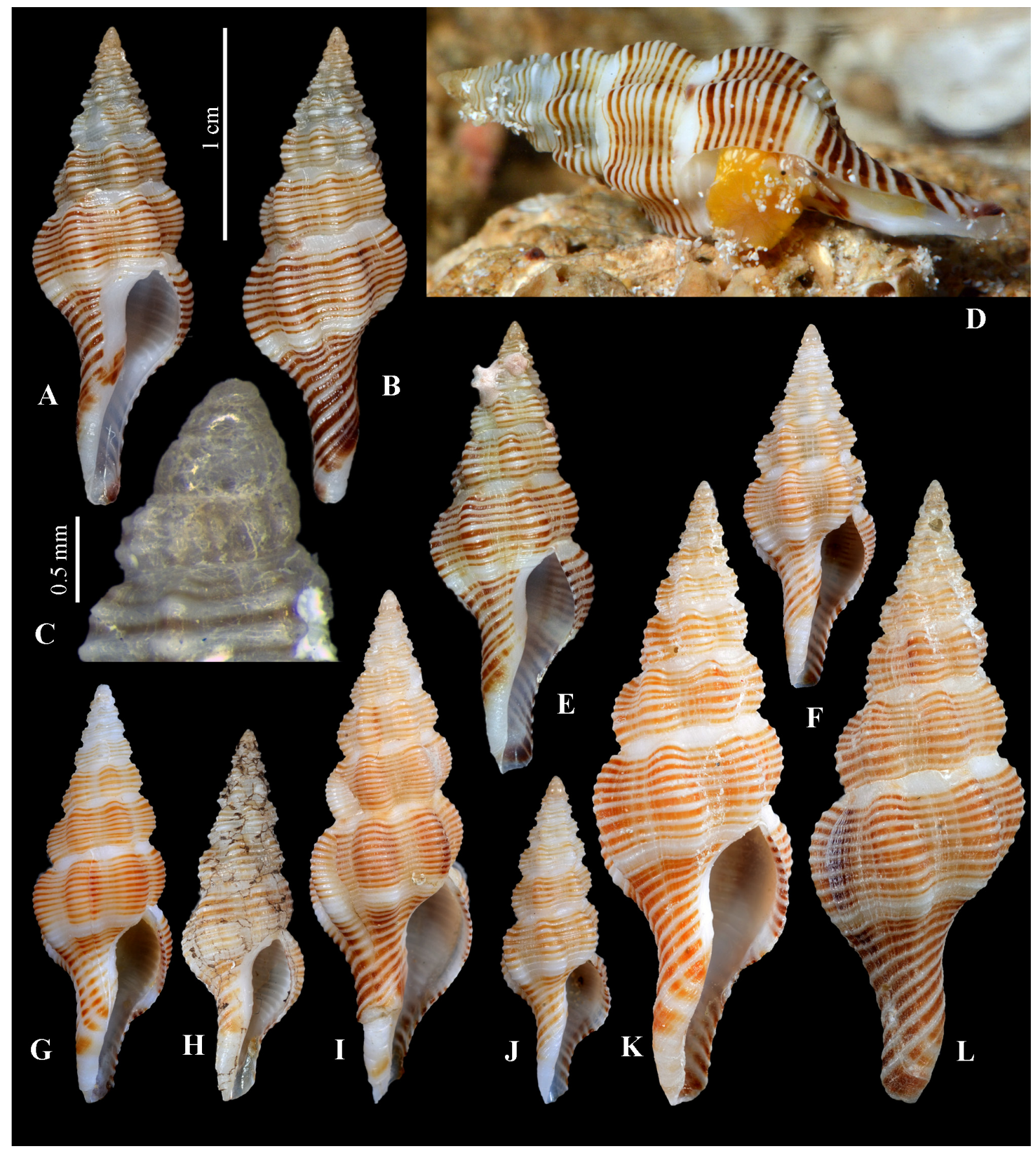

Fig. 10. Okutanius ellenae gen. et sp. nov. A-D. Holotype, New Caledonia, South of Ile des Pins, $22^{\circ} 53^{\prime}$ S, 167³5' E, 376-390 m, SL 22.7 mm, sequenced specimen (MNHN IM-2013-68819). E. Paratype from the type locality, SL $21.4 \mathrm{~mm}$, sequenced specimen (MNHN IM-2013-68876). F. New Caledonia, SMIB 5, stn DW98, $23^{\circ} 02^{\prime} \mathrm{S}, 168^{\circ} 16^{\prime} \mathrm{E}, 335 \mathrm{~m}$, SL $17.3 \mathrm{~mm}$. G. New Caledonia, BATHUS 3: stn CP804,

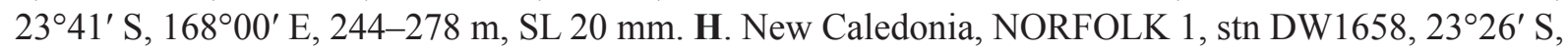
167 $50^{\prime}$ E, 320-336 m, SL 17.8 mm, radula, see Fig. 4H. I. New Caledonia, CHALCAL 2, stn DW79,

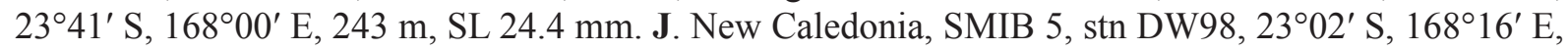

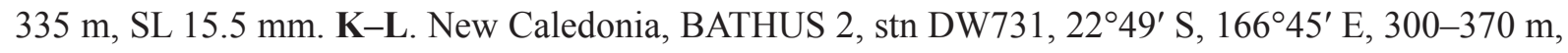
SL $29.6 \mathrm{~mm}$. All shells at the same scale. 
distinct, cusp. Central tooth small, trapezoid, with three short cusps, central longest. Narrow but distinct transverse folds of the membrane situated serially between rows of lateral teeth, reaching upper corners of central teeth.

\section{Distribution}

Coral Sea, New Caledonia to Tonga, in 245-610 m, alive in 245-450 m.

\section{Remarks}

Okutanius ellenae gen. et sp. nov. reaches $29.6 \mathrm{~mm}$ in length, showing little variation in shell shape and colouration. It resembles $O$. kuroseanus gen. et comb. nov., but is easily separated by its sculpture of even spiral cords and distinct colour pattern of alternating white cords and dark interspaces. O. kuroseanus gen. et comb. nov. reaches a much larger adult size, and is also broader at comparable size. $O$. ellenae gen. et sp. nov. also superficially resembles Granulifusus tatianae gen. et sp. nov., which differs by its short siphonal canal, strong apertural barriers, and the presence of second order spiral and axial sculpture.

Okutanius aikeni (Lussi, 2014) gen. et comb. nov.

Fig. 9H-I

Pseudolatirus aikeni Lussi, 2014: 10-11, color images of holotype.

Pseudolatirus aikeni - Mallard \& Robin 2017: 151.

\section{Type material}

\section{Holotype}

South Africa: off Park Rynie, southern KwaZulu - Natal, 110 m, SL 25.6 mm, SW 10.3 mm (NMSA W9698/T3896).

\section{Description}

Shell strong, fusiform, up to $25.6 \mathrm{~mm}$. Protoconch and uppermost teleoconch whorls eroded, protoconch conical, multispiral, of $3+$ whorls (protoconch tip broken off). Teleoconch with regularly strongly convex whorls, with medium long siphonal canal. Aperture broad, oval, with nearly smooth columella with very weak ridges in the lower part, corresponding to spiral cords not completely overlaid by callus. Spiral sculpture of unevenly spaced distinct cords of varying strength; axial ribs strong, weakly opisthocline, and closely spaced, not aligned along the spire. Background colour creamy, with two darker brown spiral bands and more dark shell base. Primary spiral cords white.

\section{Distribution}

Only known from the type locality.

\section{Remarks}

The dead collected and partially distorted holotype is the only known specimen. In ventral view it is clearly seen that the shell axis is not straight, and the strongly recurved leftwards siphon is the result of abnormal shell growth. Pending knowledge on the body and radula, we tentatively allocate this species to Okutanius gen. nov., based on shell resemblance to sequenced species of the genus. 


\section{Vermeijius gen. nov. urn:1sid:zoobank.org:act:F4D92EAC-3741-4B91-ACF0-61C1A8E2A116}

\section{Type species}

Pseudolatirus pallidus Kuroda \& Habe, 1961, here designated.

\section{Diagnosis}

Shell medium-sized, adult size from $27 \mathrm{~mm}$ ( $V$. wallacei gen. et comb. nov.) to $60 \mathrm{~mm}$ ( $V$. pallidus gen. et comb. nov.), fusiform, strong but light, usually offwhite, with evenly convex to inflated teleoconch whorls, constricted shell base and long, straight or weakly sinuous canal. Protoconch paucispiral or multispiral (1.5-3.25 whorls). Axial sculpture of distinct narrow ribs, nearly coaxial, separated by broader (1-2 times) interspaces. Spiral sculpture of distinct cords, unequal in size, covering entire shell surface, major cords nodulose at intersection with axial ribs. Aperture broad, oval, relatively small, with smooth or weakly plaited columella (plaits correspond to spiral cords covered by columellar callus). Parietal knob present in some specimens.

Operculum spans entire aperture, with terminal nucleus.

Lateral teeth with 5-7 closely spaced, slightly curved cusps and much smaller, but distinct, innermost cusp. Outer anterior corner forms long distinct 'handle', tooth outline convex at transition from cusp side to 'handle'. Central tooth small, rectangular, with three cusps. Narrow but distinct transverse folds of membrane situated serially between rows of lateral teeth.

\section{Differential diagnosis}

Vermeijius gen. nov. differs from Granulifusus and Okutanius gen. nov. in its uniformly coloured light shell with smooth or nearly smooth columella, more abruptly constricted shell base, producing a relatively narrow canal. The radula differs in having a proportionally broader central tooth. The molecular phylogenetic analysis clearly demonstrates the isolated position of the new genus as sister taxon to Chryseofusus.

\section{Etymology}

The genus is named after Geerat J. Vermeij from University of California at Davis in recognition of his immense contribution to malacology, including the taxonomy and biogeography of the Fasciolariidae. Gender masculine.

\section{Genus composition}

We include in the genus Pseudolatirus pallidus, Fusinus virginiae and Fusus? retiarius; additionally, Fusinus (Simplicifusus) guidonis Delsaerdt, 1995 from Somalia, Fusinus palmarium Hadorn \& Fraussen, 2006 from eastern Australia and Fusinus wallacei Hadorn \& Fraussen, 2006 were not sequenced, but are attributed to Vermeijius gen. nov. based on conchological similarities.

Vermeijius pallidus (Kuroda \& Habe, 1961) gen. et comb. nov.

Figs $11 \mathrm{~A}-\mathrm{E}, 12 \mathrm{~A}-\mathrm{B}$

Pseudolatirus pallidus Kuroda \& Habe in Habe, 1961: 66, pl. 33, fig. 9.

Pseudolatirus pallidus - Callomon \& Snyder 2009: 167, fig. 20. — Mallard \& Robin 2017: 154. 
Table 4. Molecular diagnoses of the species of Vermeijius gen. nov.

\begin{tabular}{|c|c|}
\hline \multicolumn{2}{|c|}{ Vermeijius virginiae comb. nov. } \\
\hline $\begin{array}{l}\text { Sequences analyzed } \\
\text { Independent combinations }\end{array}$ & 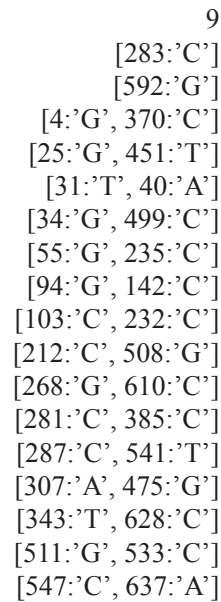 \\
\hline \multicolumn{2}{|c|}{ Vermeijius pallidus comb. nov. } \\
\hline $\begin{array}{l}\text { Sequences analyzed } \\
\text { Independent combinations }\end{array}$ & 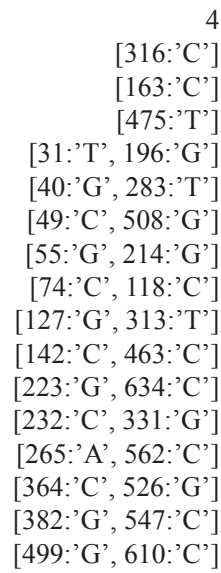 \\
\hline
\end{tabular}

\section{Molecular diagnosis}

A molecular diagnosis is given in Table 4.

\section{Type material}

\section{Holotype}

JAPAN: off Cape Ashizuri, Kochi Prefecture (NSMT Mo 40378) [not seen].

\section{Sequenced material}

SOLOMON ISLANDS: SALOMON 2: $1 \mathrm{lv}$, stn CP2290, Tetepare, $8^{\circ} 40^{\prime} \mathrm{S}, 157^{\circ} 32^{\prime} \mathrm{E}, 384-418 \mathrm{~m}$ (MNHN IM-2007-32537).

NEW CALEDONIA: CONCALIS: 1 1v, stn CP2962, Grand Passage, $18^{\circ} 59^{\prime} \mathrm{S}, 163^{\circ} 08^{\prime} \mathrm{E}, 580-647 \mathrm{~m}$ (MNHN IM-2007-35093).

SOUTH CHINA SEA: DongSha 2014, 2 lv, stn CP4128, 2042' N, 11608' E, 420-444 m (MNHN IM2013-50173, MNHN IM-2013-50174). 


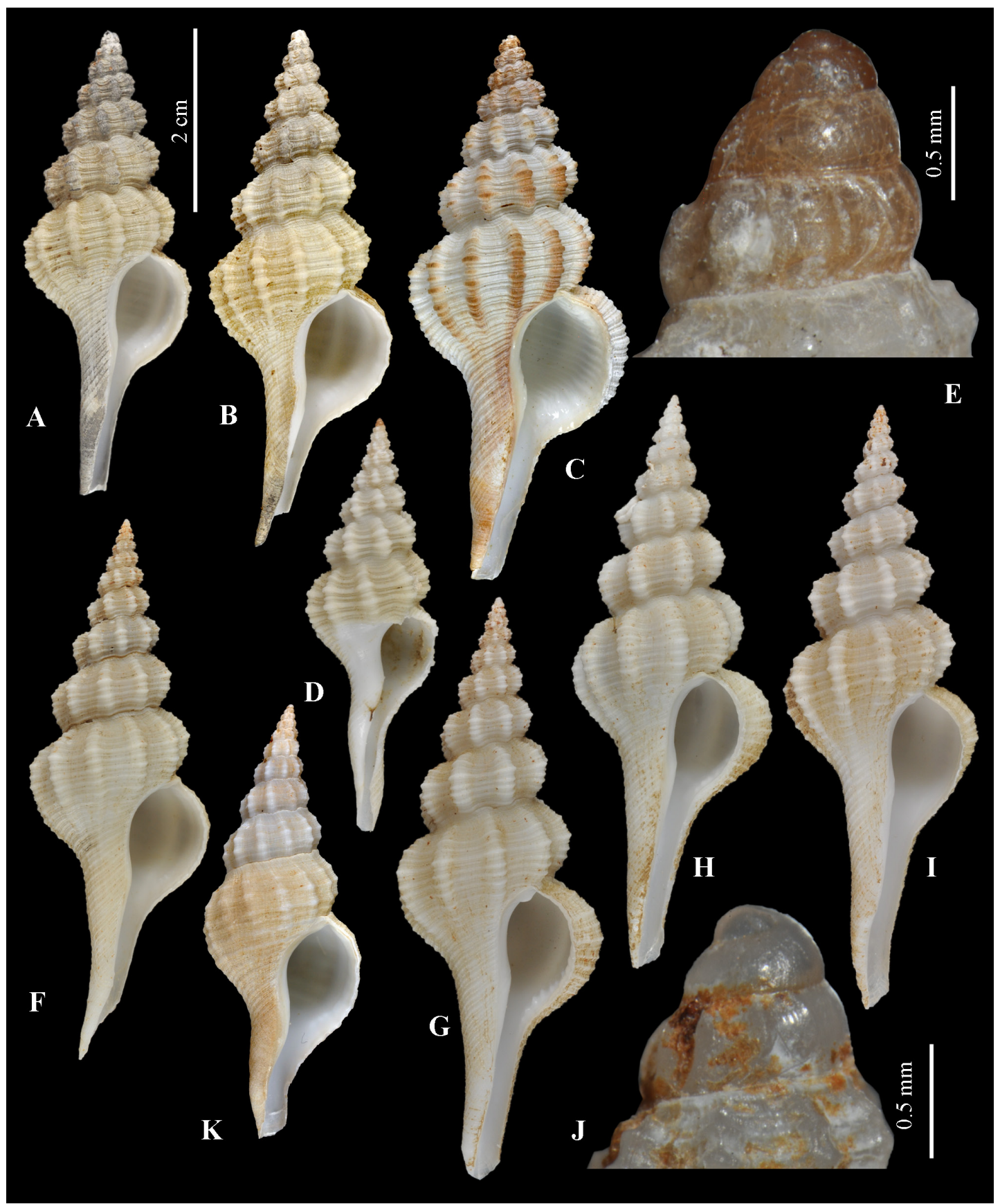

Fig. 11. Species of Vermeijius gen. nov. A-E. Vermeijius pallidus (Kuroda \& Habe, 1961) gen. et comb. nov. A-B. South China Sea, DongSha 2014, stn CP4128, 2042' N, 116 $08^{\prime}$ E, 420-444 m. A. SL $50.6 \mathrm{~mm}$ (MNHN IM-2013-50173). B. SL $56.6 \mathrm{~mm}$ (MNHN IM-2013-50174). C. Solomon Islands, SALOMON 2, stn CP2290, 08 $40^{\prime}$ S, 157³2' E, 384-418 m, SL 59.9 mm (MNHN IM-2007-32537). D-E. New Caledonia, CONCALIS, stn CP2962, Grand Passage, $18^{\circ} 59^{\prime}$ S, $163^{\circ} 08^{\prime}$ E, 580-647 m, SL 45.2 mm (MNHN IM-2007-35093). F-J. Vermeijius virginiae (Hadorn \& Fraussen, 2002) gen. et comb. nov. F. Holotype, SL 58.9 mm (MNHN IM-2000-9626). G-H. South Madagascar, ATIMO VATAE, stn CP3585, 2532' S; 44¹6' E, 549-576 m. G. SL 53.8 mm (MNHN IM-2009-15025). H. SL 52.6 mm

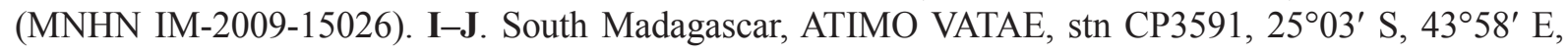
400-402 m, SL 56 mm (MNHN IM-2009-15021). K. Vermeijius retiarius (Martens, 1901) gen. et comb.

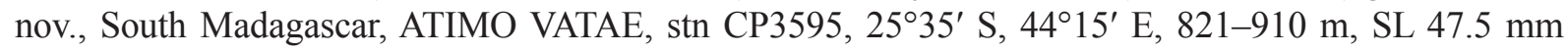
(MNHN IM-2009-15087). All specimens sequenced, except F. Figures A-D, F-I, K to the same scale. 


\section{Description}

Shell up to $60 \mathrm{~mm}$ in length, solid, fusiform, with strongly constricted base with long, nearly straight, siphonal canal, slightly inclined to left. Protoconch obtusely conical (Fig. 11E) of 3.25 whorls, with evenly convex, brown, smooth whorls, last half whorl with seven distinct, arcuate, axial ribs, protoconch/ teleoconch discontinuity distinct, marked by appearance of spiral sculpture of teleoconch. Protoconch diameter $1160 \mu \mathrm{m}$, exposed height $1100 \mu \mathrm{m}$. Teleoconch with strongly convex whorls. Aperture wide, oval, with weak columellar plicae, corresponding to primary spiral cords overlaid by callus, from nearly absent to 4-5 on lower half of columella. Spiral sculpture of unevenly arranged cords, alternating in strength, strongly nodulous at intersection with axial ribs. Axial ribs broadly spaced, narrow, not aligned along spire, 10-12 on last whorl. Growth lines usually thickened, forming reticulated structure at intersection with spiral cords. Background colour pale flesh to nearly white.

Operculum elongate, leaf-shaped, nearly spanning the entire aperture. Nucleus terminal, often abraded.

Radula (Fig. 12A-B, MNHN IM-2007-35093, SL $42.5 \mathrm{~mm}$ ) long, of about 105 rows of teeth, 30 nascent, $4.3 \mathrm{~mm}$ long ( 0.41 of AL without canal); narrow, distance between outer edges of lateral teeth about $140 \mu \mathrm{m}$ wide (1.33\% of AL without canal). Lateral teeth broad, slightly arcuate, with strongly attenuated outer anterior corner, producing long 'handle'. Lateral teeth with six, almost equal, recurved, closely
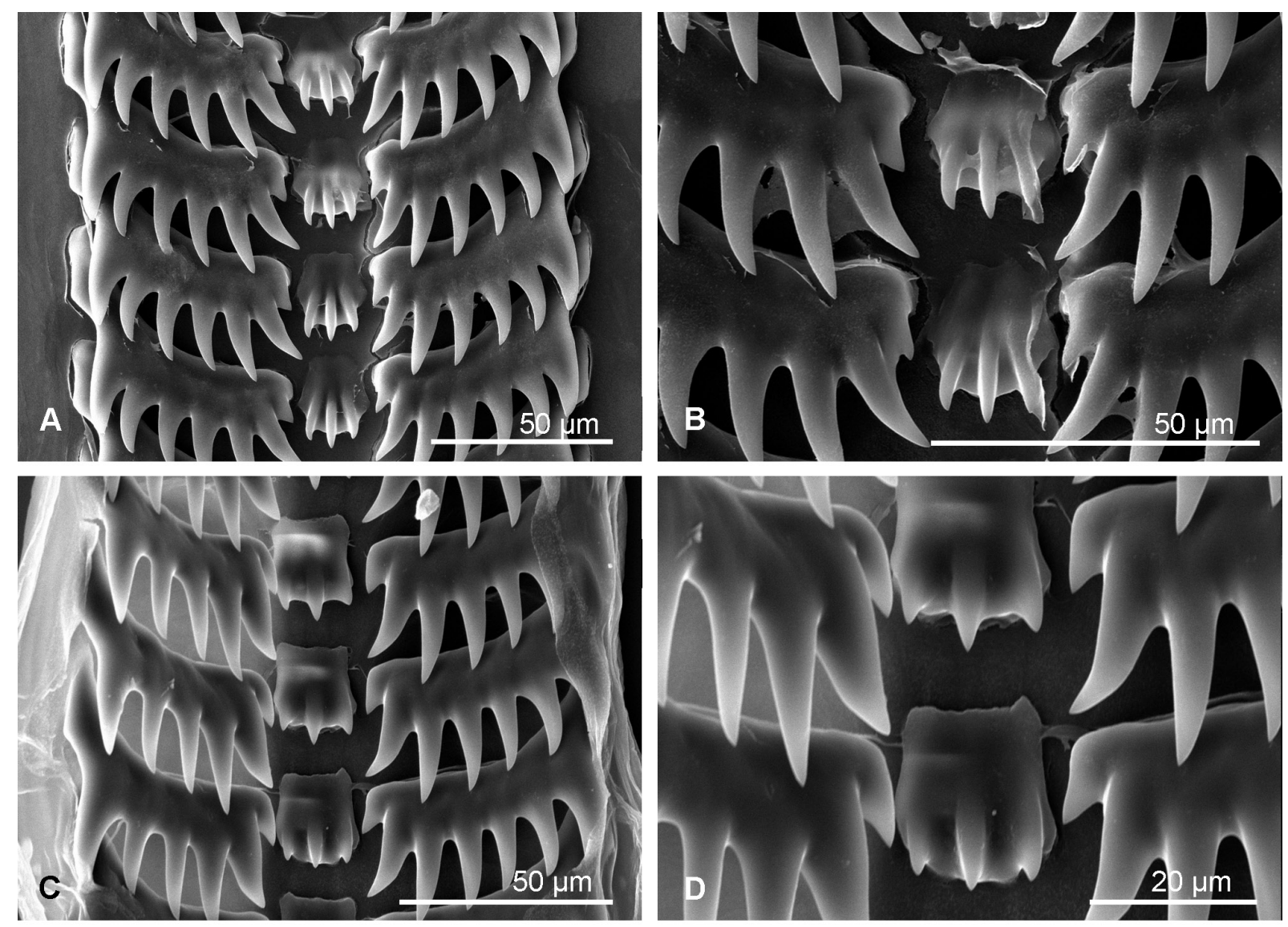

Fig. 12. Radulae of species of Vermeijius gen. nov. A-B. Vermeijius pallidus (Kuroda \& Habe, 1961) gen. et comb. nov., New Caledonia, CONCALIS, stn CP2962, Grand Passage, $18^{\circ} 59^{\prime}$ S, $163^{\circ} 08^{\prime}$ E, 580 647 m, shell, see Fig. 11D (MNHN IM-2007-35093). C-D. Vermeijius virginiae (Hadorn \& Fraussen,

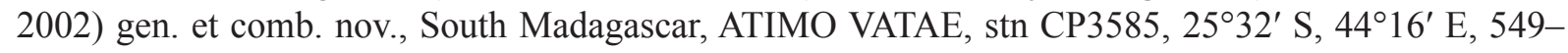
576 m (MNHN IM-2009-15030). 
spaced, major cusps and one smaller inner cusp. Teeth outline convex at transition of outer cusp side into 'handle'. Central tooth subrectangular, with three short, sharp cusps, central slightly longer. Distinct transverse folds of the membrane situated serially between rows of lateral teeth.

The radula illustrated by line drawing by Hadorn \& Fraussen (2002: fig. 22) is very similar, differing in the presence of 7 major cusps on lateral teeth versus 6 in our specimen.

\section{Distribution}

Japan to New Caledonia, in 384-647 m.

\section{Remarks}

Callomon \& Snyder (2009) discussed and illustrated the type of the species stored in NSMT (Mo 40378) and concluded that it does not match the dimensions of the holotype (52.2 vs $56.8 \mathrm{~mm}$ for the latter according to the original description); the specimen may be a figured paratype.

Vermeijius pallidus gen. et comb. nov. is somewhat similar to $V$. virginiae gen. et comb. nov.; for differences, see the description of the latter.

Vermeijius virginiae (Hadorn \& Fraussen, 2002) gen. et comb. nov.

Figs $11 \mathrm{~F}-\mathrm{J}$; $12 \mathrm{C}-\mathrm{D}$

Fusinus virginiae Hadorn \& Fraussen, 2002: 70, 72, figs 15-19.

Fusinus virginiae - Mallard \& Robin 2017: 104.

\section{Molecular diagnosis}

A molecular diagnosis is given in Table 4.

\section{Type material}

\section{Holotype}

MOZAMBIQUE CHANNEL: off Southwest Madagascar, $22^{\circ} 10^{\prime} \mathrm{S}, 43^{\circ} 05^{\prime} \mathrm{E}, 525 \mathrm{~m}$, Campagne Crevettière 1986, stn CH79 (MNHN IM-2000-9626).

\section{Sequenced material}

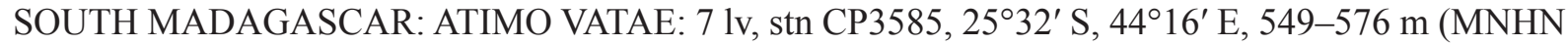
IM-2009-15020, MNHN IM-2009-15025, MNHN IM-2009-15026, MNHN IM-2009-15030, MNHN IM-2009-15031, MNHN IM-2009-15033, MNHN IM-2009-15034); 2 lv, stn CP3591, 2503' S; 435'ㄹ E, 400-402 m (MNHN IM-2009-15021, MNHN IM-2009-15084).

\section{Description}

Shell large for genus, up to $56 \mathrm{~mm}$, strong, fusiform, with strongly constricted base with long, nearly straight, siphonal canal, slightly inclined to left. Protoconch (Fig. 11J) conical of 2.25 moderately convex, smooth, greyish and semitransparent whorls, with elevated first whorl, and 2-5 indistinct axial ribs at transition to teleoconch. Protoconch/teleoconch discontinuity distinct, marked by appearance of teleoconch spiral sculpture. Protoconch diameter $960 \mu \mathrm{m}$, exposed height $800 \mu \mathrm{m}$. Teleoconch with regularly strongly convex whorls. Aperture broad, oval, columella smooth, or with 1 or 2 weak, inconspicuous plicae, corresponding to underlying spiral sculpture of preceding whorl. Spiral sculpture of unevenly spaced cords, alternating in strength, slightly nodulous at intersection with axial ribs. Growth lines usually thickened, forming reticulated structure at intersection with spiral cords. Axial ribs 
not aligned along spire, 9-11 on last whorl. Background colour pale flesh to nearly white. Operculum elongate, leaf-shaped, spanning nearly the entire aperture. Nucleus terminal.

Radula (Fig. 12C-D, MNHN IM-2009-15030, SL $51.5 \mathrm{~mm}$ ) long, of about 150 rows of teeth, 40 nascent, $4.5 \mathrm{~mm}$ long ( 0.37 of AL without canal), narrow, distance between outer edges of lateral teeth about $130 \mu \mathrm{m}$ (1.06\% of AL without canal). Lateral teeth broad, slightly arcuate, with strongly attenuated outer anterior corner, producing long 'handle'. Lateral teeth with six, almost equal, recurved, closely spaced, major cusps and one smaller inner cusp. Teeth outline convex at transition of outer cusp side into 'handle'. Central tooth subrectangular, with three very short, sharp cusps, central slightly longer. The radula illustrated by line drawing by Hadorn \& Fraussen (2002: fig. 19) is very similar to that of our specimen, but has 7 major cusps on lateral teeth versus 6 in our specimen.

\section{Distribution}

Southern Madagascar, in 400-576 m.

\section{Remarks}

The species is rather similar conchologically and is a sister to $V$. pallidus gen. et comb. nov. It differs in having fewer protoconch whorls, thinner spiral cords, forming less defined beads at intersection with axial ribs.

Vermeijius retiarius (Martens, 1901) gen. et comb. nov.

Fig. $11 \mathrm{~K}$

Fusus? retiarius Martens, 1901: 22.

Fusus? retiarius - Martens 1904: 104-105, pl. 2, fig. 4.

Fusinus retiarius - Hadorn \& Fraussen 2002: 64, 66, 68, figs 3-8, 21. - Mallard \& Robin 2017: 81.

\section{Type material}

\section{Lectotype}

SOMALIA: dd, $38.5 \times 15.7 \mathrm{~mm}$, off southern Somalia, $01^{\circ} 49^{\prime} \mathrm{N}, 45^{\circ} 29^{\prime} \mathrm{E}, 1134 \mathrm{~m}$, Valdivia, stn 256, designated by Hadorn \& Fraussen (2002) (ZMB 61012).

\section{Sequenced material}

SOUTH MADAGASCAR: ATIMO VATAE: 1 lv, stn CP3595, South of Pointe Barrow, $25^{\circ} 35^{\prime}$ S, 44ำ15' E, 821-910 m (MNHN IM-2009-15087).

\section{Description}

Shell large for genus, up to $57 \mathrm{~mm}$ in length, strong, broadly fusiform, gradually constricting to medium long, sinuous, coaxial siphonal canal. Protoconch dome-shaped, paucispiral, of 1.5 convex smooth whorls, greyish, semitransparent, with 3 weak arcuate axial ribs at transition to teleoconch. Protoconch/ teleoconch discontinuity distinct, marked by appearance of teleoconch spiral sculpture. Protoconch diameter $1000 \mu \mathrm{m}$, exposed height $700 \mu \mathrm{m}$. Teleoconch with strongly convex whorls. Aperture broad, oval, columella smooth. Spiral sculpture of moderately strong cords, beaded at intersection with axial ribs and much thinner, although distinct, secondary cords, covering interspaces between primary cords; 4-5 primary spiral cords on spire whorls, including penultimate. Axial ribs not aligned along spire, 1112 per whorl. Background colour pale flesh to nearly white. Operculum elongate, leaf-shaped, spanning nearly entire aperture. Nucleus terminal. 
Radula (Hadorn \& Fraussen 2002: fig. 21) with medium broad, arcuate, lateral teeth, with attenuated outer anterior corner, producing medium long 'handle'. Lateral teeth with five slightly recurved cusps, outermost longest, more distant from others. Additional innermost short, but distinct, cusp present. Central tooth small, subrectangular, with three very short cusps, medium longest.

\section{Distribution}

East Africa, from Somalia to South Madagascar, in 500-1134 m.

\section{Remarks}

In the COI-based tree Vermeijius retiarius gen. et comb. nov. did not cluster with $V$. pallidus gen. et comb. nov. and $V$. virginiae gen. et comb. nov., but in the three genes tree it was recovered as a sister group to the clade combining these two species. The relationships are highly supported in the Bayesian analysis, therefore we include it in Vermeijius gen. nov. It differs from the two sequenced species by a more stout shell with paucispiral protoconch.

\section{Discussion}

The current taxonomy of Fasciolariidae as well as the division of the family in three subfamilies (Fasciolariinae Gray, 1853, Fusininae Wrigley, 1927 and Peristerniinae Tryon, 1880) was suggested by Snyder (2003) based mostly on shell characters and to lesser degree on radula and anatomy, and accepted in most subsequent studies (e.g., Bouchet \& Rocroi 2005; Mallard \& Robin 2005). Nevertheless, the difficulties in allocating genera to subfamilies based on shell characters were often emphasized (e.g., Vermeij \& Snyder 2006). The situation was dramatically changed with the publication of the first molecular phylogeny of the family (Couto et al. 2016). The multigene molecular phylogeny confirmed the monophyly of the family (with the exclusion of the genera Dolicholatirus Bellardi, 1884 and Teralatirus Coomans, 1965) and its subdivision into three well supported subfamily clades, but the subfamilial allocation of many genera was changed, demonstrating the weaknesses of a conchological approach to the taxonomy of the family. Many genera of Peristerniinae, in particular, were reassigned to other subfamilies. The present analysis of a notably extended molecular dataset of Fusininae allowed the re-evaluation of the generic position of species formerly placed in Pseudolatirus, here assigned to two new genera Vermeijius gen. nov. and Okutanius gen. nov., as well as relocated to Granulifusus. The genus Vermeijius gen. nov., sister to Chryseofusus in our analysis, was recovered with high support in the three genes trees. Conchologically it differs from other 'Pseudolatirus'-like taxa, having a light coloured 'Fusinus'-type shell. Not surprisingly, all three sequenced species of Vermeijius gen. nov. were earlier described in, or attributed to, Fusinus. It should be noted that the specimen MNHN IM-200736654, erroneously identified as Fusinus virginiae in Couto et al. (2016) ends up within the genus Fusinus and represents a different, apparently still unnamed species.

Conversely, species that we re-assigned to Granulifusus and Okutanius gen. nov. demonstrate a significant overlap both in shell and anatomical characters (radula), such that the intrageneric variability exceeds the intergeneric one. The molecular data are therefore crucial for a correct generic assignment. Based on shell characters alone, it would be impossible to recognize two separate genera that appear not even closely related to each other. Similarly, the relationships revealed between the new genera (Vermeijius gen. nov. sister to Chryseofusus and Okutanius gen. nov. sister to Angulofusus) could hardly be deduced from shell characters, since these pairs of sister genera have rather different shells. At the same time, the radula of Vermeijius gen. nov. (herein) is similar to that of Chryseofusus (Couto 2016: fig. 38) in having similarly shaped laterals and trapezoid, relatively broad and short, central teeth. Similarly, the radula of Okutanius gen. nov. is rather similar to that in Angulofusus (Fedosov \& Kantor 2012: fig. 2C-D). Thus, in at least these two cases, the radular morphology is a better proxy for taxonomic decision than shell characters. 
The radulae of the species that we include in Granulifusus is variable in the shape of both the lateral and central teeth. The number of cusps on the lateral teeth varies from four to six, the innermost additional small cusp being distinct in all studied species except G. annae sp. nov. (Fig. 4E), while the shape of the central tooth varies from very narrow and long in G. annae sp. nov. to trapezoid and relatively broad in G. jeanpierrevezzaroi comb. nov. (Fig. 4C-D). The radulae of a number of species previously placed in Granulifusus illustrated by line drawings by Hadorn \& Fraussen (2005) demonstrate a similar degree of disparity. We should stress again that the relationships within the clade that corresponds to newly circumscribed Granulifusus are not fully resolved, although the clade itself is highly supported (BI 0.99). Beside, a relatively small number of species of 'traditional' Granulifusus were sequenced and included in the analysis, thus not excluding that with a more saturated dataset the polytomies will be fully resolved some new genera can be erected within Granulifusus.

One of the peculiarities of the radulae of Fasciolariidae that was never specifically discussed previously is the presence of serially arranged straight or arcuate oblique folds of the membrane that are directed from the base or middle of central teeth backwards, and extend more or less parallel to the anterior margin of the lateral teeth in interspaces between adjacent lateral teeth (marked by white arrows in Fig. 4D-E). These folds were found not only in the species of Granulifusus, Okutanius gen. nov. and Vermeijius pallidus gen. et comb. nov. studied herein but in other fasciolariids as well, particularly in Crassibougia clausicaudata (Hinds, 1844) (Stahlschmidt \& Fraussen 2012: figs 17-18), in several Fusolatirus (Snyder \& Bouchet 2006: figs 1L-M, 3K), in Angulofusus (Fedosov \& Kantor 2012: fig. 2CD), in Fusinus (Couto 2016) and at least in some members of Fasciolariinae, e.g., Polygona angulata (Röding, 1798) (Couto 2016: fig. 85). Thus these structures are found in all recognized subfamilies of Fasciolariidae. Their function or homology remain unknown, but one can speculate that they increase the rigidity of the membrane. Another insufficiently studied feature of the fasciolariid radula is that, unlike the radulae of other Buccinoidea, it is not folded longitudinally along the two axes between the central and lateral teeth. The presence of the aforementioned folds may be connected with the radular membrane being permanently unfolded. Another possibility is that the central tooth in Fasciolariidae is actually much broader than is commonly perceived, and the folds represent reduced lateral flaps of the tooth - a morphology of the rachidians found in many other neogastropods. In this case what is considered here the central tooth Fasciolariidae would, in fact, be only the central cuspate part of the tooth.

An additional unusual radular character is that the subradular membrane extends laterally well beyond the outer edges of the lateral teeth. This part of the membrane is thin, and in natural position it covers at least the lateral teeth of the radula. It is very difficult to unfold these lateral parts of the membrane during preparation of the radula for scanning electron microscopy, and it often remains overlaying the teeth as a thin film. This is seen on some of the photos (e.g. on Fig. 4B, where it covers most of the lateral teeth). This peculiarity was found in the three genera examined here in detail, as well in Angulofusus (see Fedosov \& Kantor 2012: fig. 2C-D) and may be present in other fasciolariids as well. We have not seen such subradular membrane morphology in other Buccinoidea, although many dozens of species were examined by the first author with SEM. On the contrary, usually these are the outermost edges of lateral teeth that expand beyond the limits of the membrane when it is extended.

Presently, we are witnessing an explosion of new taxa descriptions in Fasciolariidae. Out of 55 valid Recent genera accepted in WoRMS, 14 (25\%) were described before the $20^{\text {th }}$ century, $9(16.5 \%)$ were described between 1901 and 1950, 9 (16.5\%) between 1951 and 2000 and $23(42 \%)$ since 2001. This is the first work where new genera are established based on a molecular phylogenetic analysis. Many of the recently described genera have not yet been analyzed molecularly and their validity is still unconfirmed. Among genera described after 2001, Chryseofusus Hadorn \& Fraussen, 2003, Turrilatirus Vermeij \& Snyder, 2006, Pustulatirus Vermeij \& Snyder, 2006, Amiantofusus Fraussen, Kantor \& Hadorn, 2007 and 
Angulofusus Fedosov \& Kantor, 2012, proved to be monophyletic in the analysis of Couto et al. (2016). Other genera (e.g., Fusolatirus Kuroda \& Habe, 1971) proved to be paraphyletic in their accepted scope; the conchologically distinctive Cyrtulus Hinds, 1843 was unequivocally demonstrated to be merely a species of Fusinus and Pseudolatirus, as demonstrated here, is to be restricted to fossil species only. This suggests that establishing new genera solely on conchological characters is risky and should be avoided unless necessary. The use of morphological characters (radula), although strongly recommended, does not necessarily provide solid evidence for phylogenetic distinctiveness of a lineage in question.

\section{Acknowledgments}

The MNHN Indo-Pacific material originates from a series of expeditions and workshops, conducted in the context of the 'Our Planet Reviewed' programme with Pro-Natura International (SANTO 2006 to Vanuatu; ATIMO VATAE to South Madagascar in partnership with Université de Tuléar; KAVIENG 2014 to Papua-New Guinea, in partnership with University of Papua-New Guinea and the National Fisheries College), of the 'Tropical Deep-Sea Benthos' programme with Institut de Recherche pour le Développement (CONCALIS, TERRASSES, NORFOLK 2, EXBODI, EBISCO, TARASOC, SALOMON 2 and SALOMONBOA3 cruises in the South Pacific; PANGLAO 2005 and AURORA 2007 cruises to the Philippines, in partnership with University San Carlos and the Philippines Bureau of Fisheries and Aquatic Resources). The organizers thank the Total Foundation, Prince Albert II of Monaco Foundation, Stavros Niarchos Foundation, Foundation EDF, Vinci Entrepose Contracting and Richard Lounsbery Foundation for their support to these expeditions. Virginie Héros, Philippe Maestrati, Pierre Lozouet, Nicolas Puillandre helped collect and process specimens in the field, and Barbara Buge curated the molecular material in the lab. All expeditions operated under the regulations then in force in the countries in question and satisfy the conditions set by the Nagoya Protocol for access to genetical resources.

We are very grateful to Geerat J. Vermeij and a second anonymous reviewer for their valuable suggestions and comments, that improved the manuscript.

The contribution of Yu.I. Kantor and A.F. Fedosov was funded by the grant No. 16-14-10118 from the Russian Science Foundation (principal investigator Yu.I.Kantor). The molecular and SEM studies were conducted using Joint Usage Center 'Instrumental methods in ecology' at the A.N. Severtsov Institute of Ecology and Evolution, Russian Academy of Sciences and it is a pleasure to thank Anna Neretina and Alexei Nekrasov for their assistance with scanning electron microscope, and Ilya Meschersky for assistance with the sequencing facilities.

The molecular phylogenetic studies in MNHN were supported by the 'Service de Systématique Moléculaire' (UMS 2700 CNRS-MNHN), the Actions Transversales du Muséum Barcode (principal investigators: Sarah Samadi and Jean-Noël Labat, MNHN).

\section{References}

Azuma M. 1960. A Catalogue of the Shell-bearing Mollusca of Okinoshima, Kashiwajima and the adjacent Area (Tosa Province) Shikoku, Japan. Tosa-Wan Kai-Rui Bun-Ken Kan-Kō-Kai. Tosa Bay Malacological Literature Publication Committee.

Bellardi L. 1884. I molluschi dei terreni terziarii del Piemonte e della Liguria. Parte 4. Fasciolariidae e Turbinellidae. Memorie della Reale Accademia delle Scienze di Torino, Serie Seconda 37: 1-62 (1886). [Also issued as a preprint 1884].

Bouchet P. \& Rocroi J-P. 2005. Classification and nomenclator of gastropod families. Malacologia 47: $1-397$. 
Callomon P. \& Snyder M.A. 2009. On the Genus Fusinus in Japan V: further species, an unnamed form and discussion. Venus, Japanese Journal of Malacology 67 (3-4): 159-172.

Colgan D.J., Ponder W.F. \& Eggler P.E. 2000. Gastropod evolutionary rates and phylogenetic relationships assessed using partial 28S rDNA and histone H3 sequences. Zoologica Scripta 29: 29-63. https://doi.org/10.1046/j.1463-6409.2000.00021.x

Cossignani T. 2017. Pseudolatirus jeanpierrevezzaroi nuova specie dal Vietnam. Malacologia, Mostra Mondiale 95: 14-15.

Cossmann M. 1901. Essais de Paléoconchologie comparée. 4. Published by the author, Paris. https://doi.org/10.5962/bhl.title.52314

Couto D.R. 2016. A phylogenetic Analysis of Fasciolariidae (Gastropoda: Buccinoidea). PhD thesis, Museu de Zoologia da Universidade de São Paulo, Brazil.

Couto D.R., Bouchet P., Kantor Y.I., Simone L.R.L. \& Giribet G. 2016. Amultilocus molecular phylogeny of Fasciolariidae (Neogastropoda: Buccinoidea). Molecular Phylogenetics and Evolution 99: 309-322. https://doi.org/10.1016/j.ympev.2016.03.025

d'Orbigny A. 1850-1852. Prodrome de paléontologie stratigraphique universelle des animaux mollusques \& rayonnés, faisant suite au cours élémentaire de paléontologie et de géologie stratigraphiques. 3 volumes. Victor Masson, Paris.

Fedosov A.E. \& Kantor Y.I. 2012. A new species and genus of enigmatic turriform Fasciolariidae from the Central Indo-Pacific (Gastropoda: Neogastropoda). Archiv für Molluskenkunde 141: 137-144. https://doi.org/10.1127/arch.moll/1869-0963/141/137-144

Felsenstein J. 1985. Confidence limits on phylogenies: an approach using the bootstrap. Evolution 39: 783-791. https://doi.org/10.1111/j.1558-5646.1985.tb00420.x

Finlay H.J. 1927. New specific names for Austral Mollusca. Transactions of the New Zealand Institute 57: 488-533.

Folmer O., Black M., Hoeh W., Lutz R. \& Vrijenhoek R. 1994. DNA primers for amplification of mitochondrial cytochrome c oxidase subunit I from diverse metazoan invertebrates. Molecular Marine Biology and Biotechnology 3: 294-299.

Galindo L.A., Puillandre N., Strong E.E. \& Bouchet P. 2014. Using microwaves to prepare gastropods for DNA barcoding. Molecular Ecology Resources 14: 700-705. https://doi.org/10.1111/1755-0998.12231

Habe T. 1961. Coloured Illustrations of the Shells of Japan. Vol. II. Hoikusha, Osaka.

Habe T. 1962. Coloured Illustrations of the Shells of Japan. Vol. II, $2^{\text {nd }}$ edition. Hoikusha, Osaka.

Hadorn R. \& Fraussen K. 2002. Two new Fusinus from East Africa (Gastropoda: Fasciolariidae). [Dos nuevos Fusinus de África oriental (Gastropoda: Fasciolariidae)]. Iberus 20: 63-72.

Hadorn R. \& Fraussen K. 2005. Revision of the genus Granulifusus Kuroda \& Habe, 1954, with description of some new species. Archiv für Molluskenkunde 134: 129-171. https://doi.org/10.1127/arch.moll/0003-9284/134/129-171

Hall T.A. 1999. BioEdit: a user-friendly biological sequence alignment editor and analysis program for Windows 95/98/NT. Nucleic Acids Symposium Series 41: 95-98.

Hasegawa K. \& Saito H. 1995. Illustrations and annotated Checklist of the molluscan Type Specimens contained in the Sakurai Collection in the National Science Museum, Tōkyō. National Science Museum, Tōkyō. 
Héros V., Strong E.E. \& Bouchet P. (eds). 2016. Tropical Deep-Sea Benthos. Volume 29. Mémoires du Muséum national d'Histoire naturelle 208, Muséum national d'Histoire naturelle, Paris.

Higo S., Callomon P. \& Gotō Y. 2001. Catalogue and Bibliography of the marine Shell-bearing Mollusca of Japan. Gastropoda, Bivalvia, Polyplacophora, Scaphopoda. Type Figures. Elle Scientific Publications, Ōsaka-fu, Japan.

Huelsenbeck J.P. \& Ronquist F. 2001. MRBAYES: Bayesian inference of phylogenic trees. Bioinformatics 17: 754-755. https://doi.org/10.1093/bioinformatics/17.8.754

Jovelin R. \& Justine J.-L. 2001. Phylogenetic relationships within the Polyopisthocotylean monogeneans (Plathyhelminthes) inferred from partial 28S rDNA sequences. International Journal of Parasitology 31 (4): 393-401. https://doi.org/10.1016/s0020-7519(01)00114-x

Kaicher S.D. 1986. Card Catalogue of World-Wide Shells. Pack 46. Fasciolariidae II. Kaicher, St. Petersburg FL.

Kantor Yu.I. \& Puillandre N. 2012. Evolution of the radular apparatus in Conoidea (Gastropoda: Neogastropoda) as inferred from a molecular phylogeny. Malacologia 55 (1): 55-90. https://doi.org/10.4002/040.055.0105

Koperberg E.J. 1931. Jungtertiäre und Quartäre Mollusken von Timor. $2^{\mathrm{e}}$ Nederlandsche TimorExpeditie 1916, 7. Jaarboek van het Mijnwezen in Nederlandsch Oost-Indië, Verhandelingen 1930. [Also published as a thesis, 1931].

Kosuge S. 1979. Report on the Mollusca on Guyots from the central Pacific collected by $2^{\text {nd }}$ and $3^{\text {rd }}$ cruises of R/V Kaiyomaru in 1972 and 73 with descriptions of twelve new species. Bulletin of the Institute of Malacology, Tōkyō 1: 24-35.

Lussi M. 2014. Description of seven new species of Fasciolariidae from off the eastern seaboard of South Africa with notes on Granulifusus rubrolineatus (Sowerby II, 1870) - (Gastropoda: Neogastropoda: Buccinoidea). Malacologia Mostra Mondiale 84: 3-12.

MacNeil F.S. 1961. Tertiary and Quaternary Gastropoda of Okinawa. United States Geological Survey Professional Paper 339, US Government Printing Office, Washington. [Imprinted 1960 but distributed March 17, 1961].

Mallard D. \& Robin A. 2005. Fasciolariidae. Museum du Coquillage, Les Sables d'Olonne.

Mallard D. \& Robin A. 2017. Recent Fasciolariidae. Association Francaise de Conchiologie, Paris and Conchbooks, Harxheim.

Marais J.P. \& Kilburn R.N. 2010. Fasciolariidae In: Marais A.P. \& Seccombe A.D. (eds) Indentification Guide to the Seashells of South Africa, volume 1. Centre for Molluscan Studies, Groenklouf, South Africa.

Martens E. von. 1901. Neue Meer-Conchylien von der deutschen Tiefsee-Expedition unter der Leitung von Prof. Chun. Sitzungsberichte der Gesellschaft naturforschender Freunde zu Berlin: 14-26.

Martens E. von. 1904. Die beschalten Gasteropoden der deutschen Tiefsee-Expedition, 1898-1899. A. Systematisch-geographischer Teil. Wissenschaftliche Ergebnisse der deutschen Tiefsee-Expedition auf dem Dampfer ,Valdivia“ 1898-1899 7: 1-180. Verlag Gustav Fischer, Jena.

https://doi.org/10.5962/bhl.title.2171

Miller M.A., Pfeiffer W. \& Schwartz T. 2010. Creating the CIPRES Science Gateway for inference of large phylogenetic trees. In: Proceedings of the Gateway Computing Environments Workshop (GCE), 14 Nov. 2010, LA: 1-8. Institute of Electrical and Electronics Engineers, New Orleans. 
Okutani T. 1975. Glimpse of benthic molluscan fauna occupying the submarine bank, Kurose, near Hachijō Island, Japan. Venus, Japanese Journal of Malacology 33: 185-205.

Okutani T. (ed.) 2000. Marine Molluscs in Japan. Tokai University Press, Japan.

Okutani T. \& Sakurai K. 1964. A new exquisite fasciolarid gastropod, Pseudolatirus kurodai, n. sp., dedicated to Dr. T. Kuroda for the seventy-seventh anniversary of his birth. Venus, Japanese Journal of Malacology 23: 37-39.

Okutani T. \& Sakurai K. 1966. Corrigendum for the type locality of Pseudolatirus kurodai Okutani and Sakurai, 1964. Venus, Japanese Journal of Malacology 24: 358.

Poppe G.T. \& Tagaro S.P. 2006. A new Fusinus from the Philippines. Visaya 1: 48-50.

Poppe G.T. 2008. Philippine Marine Mollusks. Vol. 2. (Gastropoda-Part 2). Conchbooks, Harxheim.

Puillandre P., Lambert A., Brouillet S. \& Achaz G. 2012. ABGD, Automatic Barcode Gap Discovery for primary species delimitation. Molecular Ecology 21: 1864-1877.

https://doi.org/10.1111/j.1365-294X.2011.05239.x

Rambaut A., Suchard M.A., Xie D. \& Drummond A.J. 2014. Traver v. 1.6. Available from http://beast.bio.ed.ac.uk/Tracer [accessed 19 Mar. 2018].

Richer de Forges B. 1990. Explorations for bathyal fauna in the New Caledonian economic zone. ). In: Crosnier A. (ed.) Résultats des Campagnes MUSORSTOM. Volume 6. Mémoires du Muséum national d'Histoire naturelle A 145: 9-54.

Richer de Forges B. 1991. Les fonds meubles des lagons de Nouvelle-Calédonie: généralités et échantillonnage par dragages. In: Richer de Forges B. (ed.) Le benthos des fonds meubles des lagons de Nouvelle-Calédonie. Volume 1: 7-148. Etudes et Thèses, MUSORSTOM, Paris.

Richer de Forges B. 1993. Campagnes d'exploration de la faune bathyale faites depuis mai 1989 dans la zone économique de la Nouvelle-Calédonie. Listes des stations. In: Crosnier A. (ed.) Résultats des Campagnes MUSORSTOM. Volume 10. Mémoires du Muséum national d'Histoire naturelle 156: $27-$ 32.

Richer de Forges B. \& Chevillon C. 1996. Les campagnes d'échantillonnage du benthos bathyal en Nouvelle-Calédonie, en 1993 et 1994 (BATHUS 1 à 4, SMIB 8 et HALIPRO 1). In: Crosnier A. (ed.) Résultats des Campagnes MUSORSTOM. Volume 15. Mémoires du Muséum national d'Histoire naturelle 168: 33-53.

Richer de Forges B. \& Menou J.L. 1993. La campagne MUSORSTOM 7 dans la zone économique des iles Wallis et Futuna. Compte rendu et liste des stations. In: Crosnier A. (ed.) Résultats des campagnes MUSORSTOM. Volume 10. Mémoires du Muséum national d'Histoire naturelle 156: 9-25.

Richer de Forges B., Faliex E. \& Menou J.L. 1996. La campagne MUSORSTOM 8 dans l'archipel de Vanuatu. Compte rendu et liste des stations. In: Crosnier A. (ed.) Résultats des Campagnes MUSORSTOM. Volume 15. Mémoires du Muséum national d'Histoire naturelle 168: 9-32.

Richer de Forges B., Poupin J. \& Laboute P. 1999. La campagne MUSORSTOM 9 dans l'archipel des iles Marquises (Polynésie française). Compte rendu et liste des stations. In: Crosnier A. (ed.) Résultats des Campagnes MUSORSTOM. Volume 20. Mémoires du Muséum national d'Histoire naturelle 180: 9-29.

Richer de Forges B., Bouchet P., Dayrat B., Waren A. \& Philippe J.S. 2000a. La campagne BORDAU 1 sur la ride de Lau (iles Fidji). Compte rendu et liste des stations. In: Crosnier A. (ed.) Résultats des Campagnes MUSORSTOM. Volume 21. Mémoires du Muséum national d'Histoire naturelle 184: $25-38$. 
Richer de Forges B., Newell P., Schlacher-Hoenlinger M., Schlacher T., Nating D., Cesa F. \& Bouchet P. 2000b. La campagne MUSORSTOM 10 dans l'archipel des iles Fidji. Compte rendu et liste des stations. In: Crosnier A. (ed.) Résultats des Campagnes MUSORSTOM. Volume 21. Mémoires du Muséum national d'Histoire naturelle 184: 9-23.

Robba E., Sartono S., Violanti D. \& Erba E. 1989. Early Pleistocene gastropods from Timor (Indonesia). Memorie di Scienze Geologiche [Padova] 41: 61-113.

Sabelli B., Giannuzzi-Savelli R. \& Bedulli D. 1990. Catalogo annotato dei Molluschi marini del Mediterraneo. Volume 1. Libreria Naturalistica Bolognese, Società Italiana di Malacologia. Bologna.

Sarkar I.N., Planet P.J. \& DeSalle R. 2008. CAOS software for use in character-based DNA barcoding. Molecular Ecology Resources 8: 1256-1259. https://doi.org/10.1111/j.1755-0998.2008.02235.x

Shutō T. 1969. Neogene Gastropods from Panay Island, the Philippines (Contributions to the Geology and Palaeontology of southeast Asia, LXVIII). Memoirs of the Faculty of Science, Series D, Geology 19, Kyūshū University, Kyūshū.

Snyder M.A. 2003. Catalogue of the marine Gastropod Family Fasciolariidae. Academy of Natural Sciences of Philadelphia, special publication 21. Academy of Natural Sciences, Philadelphia.

Snyder M.A. \& Bouchet P. 2006. New species and new records of deep-water Fusolatirus (Neogastropoda: Fasciolariidae) from the west Pacific. Journal of Conchology 39: 1-12.

Springsteen F.J. \& Leobrera F.M. 1986. Shells of the Philippines. Carfel Seashell Museum, Manila.

Stahlschmidt P. \& Fraussen K. 2012. Crassibougia, a new genus for Fusus clausicaudatus Hinds, 1844, from South Africa, with description of a new species (Gastropoda: Fasciolariidae). Miscellanea Malacologica 5: 85-93.

Stamatakis A. 2006. RAxML-VI-HPC: maximum likelihood-based phylogenetic analyses with thousands of taxa and mixed models. Bioinformatics 22: 2688-2690. https://doi.org/10.1093/bioinformatics/bt1446

Thach N.N. 2007. Recently Collected Shells of Vietnam. L'Informatore Piceno, Ancona.

Vermeij G.J. \& Snyder M.A. 2006. Shell characters and taxonomy of Latirus and related fasciolariid groups. Journal of Molluscan Studies 72: 413-424. https://doi.org/10.1093/mollus/eyl020

Wanner J. \& Hahn E. 1935. Miocäne Mollusken aus der Landschaft Rembang. Zeitschrift der deutschen geologischen Gesellschaft 87: 222-273.

Manuscript received: 25 July 2017

Manuscript accepted: 5 December 2017

Published on: 7 May 2018

Topic editor: Rudy Jocqué

Section editor: Kurt Jordaens

Desk editor: Kristiaan Hoedemakers

Printed versions of all papers are also deposited in the libraries of the institutes that are members of the EJT consortium: Muséum national d'Histoire naturelle, Paris, France; Botanic Garden Meise, Belgium; Royal Museum for Central Africa, Tervuren, Belgium; Natural History Museum, London, United Kingdom; Royal Belgian Institute of Natural Sciences, Brussels, Belgium; Natural History Museum of Denmark, Copenhagen, Denmark; Naturalis Biodiversity Center, Leiden, the Netherlands; Museo Nacional de Ciencias Naturales-CSIC, Madrid, Spain; Real Jardín Botánico de Madrid CSIC, Spain; Zoological Research Museum Alexander Koenig, Bonn, Germany. 